Aus der Klinik für Pädiatrische Kardiologie und Intensivmedizin

(Prof. Dr. med. Paul)

der Medizinischen Fakultät der Universität Göttingen

\title{
Validierung des Totalen Rechts/Links-Volumen-Index als MRT-Parameter zur Evaluation des Schweregrads der Ebstein Anomalie - eine Follow-Up-Studie
}

\author{
INAUGURAL-DISSERTATION \\ zur Erlangung des Doktorgrades \\ der Medizinischen Fakultät der \\ Georg-August-Universität zu Göttingen
}

\author{
vorgelegt von \\ Larissa Kathrin Schenk \\ aus \\ Heidelberg
}


Dekan:

Betreuungsausschuss

Betreuer/in:

Ko-Betreuer/in:

Prüfungskommission

Referent/in:

Ko-Referent/in:

Drittreferent/in:

Datum der mündlichen Prüfung:
Prof. Dr. W. Brück

PD Dr. M. Steinmetz

Prof. Dr. Dr. A. Schuster

PD Dr. med. M. Steinmetz

Prof. Dr. Dr. A. Schuster

Prof. Dr. M. Schön

07.07.2021 
Hiermit erkläre ich, die Dissertation mit dem Titel "Validierung des Totalen Rechts/Links-Volumen-Index als MRT-Parameter zur Evaluation des Schweregrads der Ebstein-Anomalie - eine Follow-Up-Studie" eigenständig angefertigt und keine anderen als die von mir angegebenen Quellen und Hilfsmittel verwendet zu haben.

Göttingen, den 


\section{Inhaltsverzeichnis}

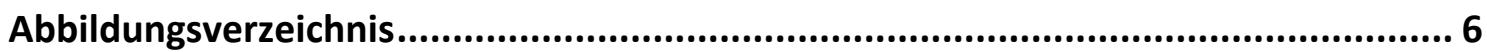

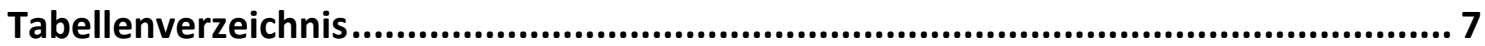

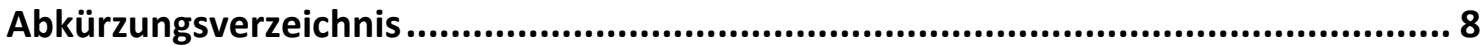

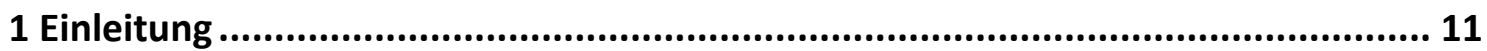

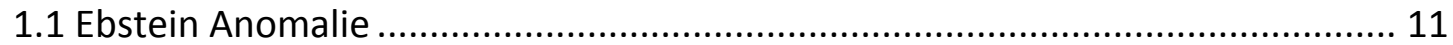

1.2 Kardiale Magnetresonanztomographie ......................................................... 23

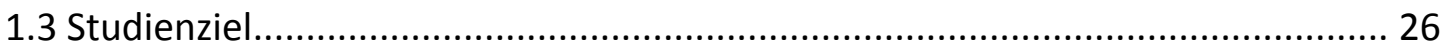

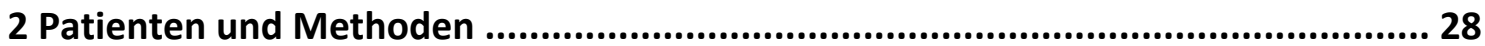

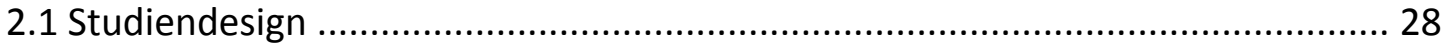

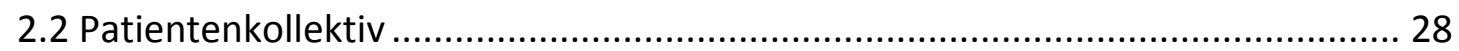

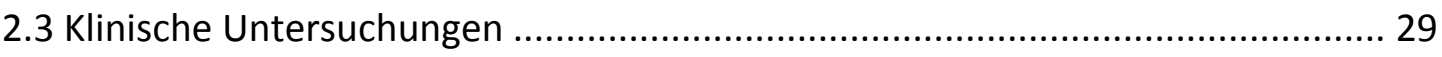

2.4 Kardiale Magnetresonanztomographie ........................................................ 33

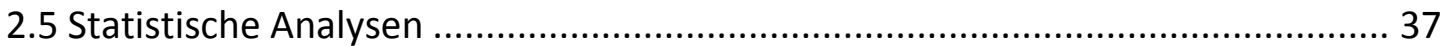

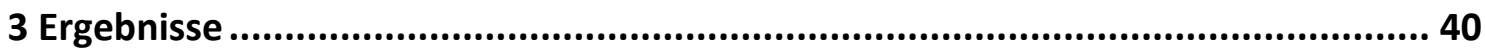

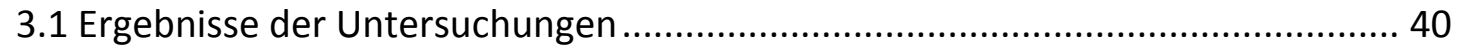

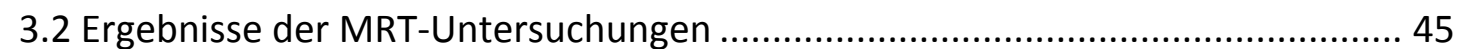

3.3 Der Totale Rechts/Links-Volumen-Index 2013 und 2016 ............................... 47

3.4 Der Totale Rechts/Links-Volumen-Index als prognostischer Parameter zur Risikostratifizierung für das Auftreten kardialer Ereignisse..................................... 54

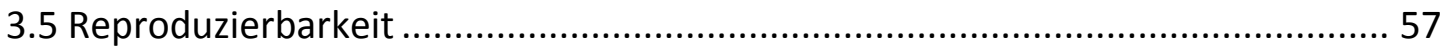

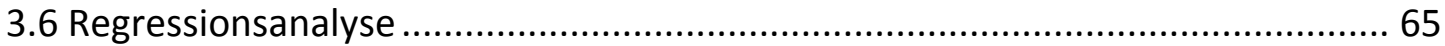

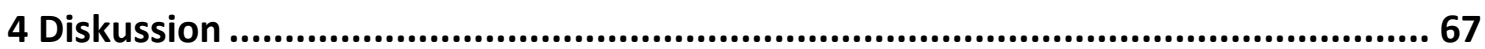

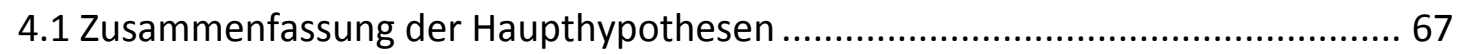

4.2 Veränderungen des Totalen Rechts/Links-Volumen-Index im Bezug auf den klinischen Zustand der Patienten.

4.3 Beurteilung des Totalen Rechts/Links-Volumen-Index als Verlaufsparameter und als Parameter zur Risikostratifizierung für das Auftreten kardialer Ereignisse.......... 72

4.4 Der Nutzen des Totalen Rechts/Links-Volumen-Index als Parameter für das Treffen therapeutischer Entscheidungen

4.5 Bewertung der Reproduzierbarkeit der MRT-Ergebnisse und des Totalen Rechts/Links-Volumen-Index 
4.6 Regressionsanalyse zur Beurteilung des Totalen Rechts/Links-Volumen-Index als Vorhersageparameter für Änderungen in den klinischen Parametern 82

4.7 Limitationen der Studie 82

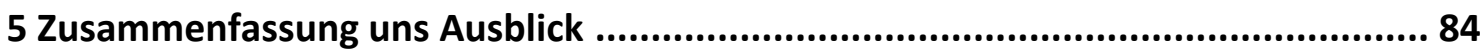

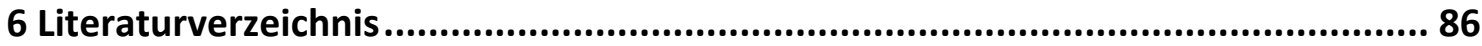




\section{Abbildungsverzeichnis}

Abbildung 1: Echokardiographische Einteilung der Ebstein Anomalie gemäß Celermajer im enddiastolischen Vier-Kammer-Blick (Celermajer et al. 1992; Hösch 2015). 20

Abbildung 2: Anatomische Einteilung der Ebstein Anomalie gemäß Carpentier et al. (1988).

Abbildung 3: Illustration der Berechnung des Totalen Rechts/Links-Volumen-Index in axialer Bildebene mit Formel (Hösch et al. 2014).

Abbildung 4: Dreidimensionale Reproduktion der Ebstein Anomalie (Yalonetsky et al. 2011) und Segmentierungsregeln der cMRT-Bilder (Hösch et al. 2014) in der kurzen Achse und in axialer Ausrichtung.

Abbildung 5: Segmentierung der einzelnen Kompartimente des Herzens und vereinfachte Kontureneinzeichnung zur Ermittlung des Totalen Rechts/Links-VolumenIndex.

Abbildung 6: Ergebnisse der Pearson-Korrelationen des laborchemischen Parameters BNP-Wert mit dem Totalen Rechts/Links-Volumen-Index aus dem Jahr 2013 und aus dem Jahr 2016.

Abbildung 7: Ergebnisse der Pearson-Korrelationen des EKG-Parameters QRS-Zeit mit dem Totalen Rechts/Links-Volumen-Index aus dem Jahr 2013 und aus dem Jahr 2016.

Abbildung 8: Ergebnisse der Korrelationen des spiroergometrischen Parameters max. $\mathrm{VO}_{2} / \mathrm{kg}$ mit dem Totalen Rechts/Links-Volumen-Index aus dem Jahr 2013 und aus dem Jahr 2016.

Abbildung 9: Ergebnisse der Pearson-Korrelationen des spiroergometrischen Parameters max. VE/VCO ${ }_{2}$ mit dem Totalen Rechts/Links-Volumen-Index aus dem Jahr 2013 und aus dem Jahr 2016.

Abbildung 10: Ergebnisse der Pearson-Korrelationen des echokardiographischen Parameters TAPSE mit dem Totalen Rechts/Links-Volumen-Index aus dem Jahr 2013 und aus dem Jahr 2016.

Abbildung 11: Ergebnisse der Überlebenszeitanalyse nach Kaplan-Maier. 56

Abbildung 12: Bland-Altman-Diagramm für die Intraobserver-Variabilität des Totalen Rechts/Links-Volumen-Index.

Abbildung 13: Bland-Altman-Diagramm für die Interobserver-Variabilität des Totalen Rechts/Links-Volumen-Index.

Abbildung 14: Bland-Altman-Diagramm für die Errechnung des Totalen Rechts/LinksVolumen-Index mittels Einzelkammersegmentierung im Vergleich zur Errechnung mittels Gesamtkontureneinzeichnung der rechten und linken Herzhälfte. 


\section{Tabellenverzeichnis}

Tabelle 1: Patientenmerkmale und klinische Parameter 2016.

Tabelle 2: Patientenmerkmale und klinische Parameter 2013 und 2016 im Vergleich. 43

Tabelle 3: Die berechnetes cMRT Funktionsparameter und Herzkammervolumina von 2013 und $2016 \mathrm{im}$ Vergleich.

Tabelle 4: Der Totale Rechts/Links-Volumen-Index aller Studienteilnehmer 2013 und 2016 im Vergleich.

Tabelle 5: Anzahl, Mittelwert und Standardabweichung des Totalen Rechts/Links-

Volumen-Index 2013 und 2016.

Tabelle 6: Pearson-Korrelationen des Totalen Rechts/Links-Volumen-Index mit klinischen Parametern 2013 und 2016

Tabelle 7: Vergleich der Totalen Rechts/Links-Volumen-Indizes der Patienten mit und ohne aufgetretene kardiale Ereignisse innerhalb von drei Jahren.

Tabelle 8: Vergleich der Anzahl an Patienten mit und ohne aufgetretene kardiale Ereignisse in drei Jahren.

Tabelle 9: Variationskoeffizient (CoV) für die Intraobserver- und die InterobserverVariabilität 2016.

Tabelle 10: Ergebnisse für die Intraobserver-Variabilität 2016; Der IntraklassenKorrelationskoeffizient (ICC)

Tabelle 11: Ergebnisse für die Interobserver-Variabilität 2016; Der Intraklassen-

Korrelationskoeffizient (ICC).

Tabelle 12: Ergebnisse der Bland-Altman-Analyse für die Intraobserver-Variabilität 2016.

Tabelle 13: Ergebnisse der Bland-Altman-Analyse für die Interobserver-Variabilität 2016.

Tabelle 14: Ergebnisse der multiplen Regressionsanalyse.

Tabelle 15: Vergleich der Intraobserver-Variabilität der biventrikulären Volumina aus MRT-Untersuchungen verschiedener Studien (CoV).

Tabelle 16: Vergleich der Interobserver-Variabilität der biventrikulären Volumina aus MRT-Untersuchungen verschiedener Studien (CoV). 


\section{Abkürzungsverzeichnis}

\begin{tabular}{|c|c|}
\hline $4 \mathrm{CV}$ & Vier-Kammer-Blick \\
\hline aRV & atrialisierter rechter Ventrikel \\
\hline ASD & Atriumseptumdefekt \\
\hline BMI & body mass index \\
\hline BNP & brain natriuretic peptide \\
\hline BSA & body surface area \\
\hline DGPK & Deutsche Gesellschaft für Pädiatrische Kardiologie \\
\hline EA & Ebstein Anomalie \\
\hline EDV & enddiastolisches Volumen \\
\hline$E F$ (n.S.) & Ejektionsfraktion (nach Simpson) \\
\hline EKG & Elektrokardiogramm \\
\hline EPU & Elektrophysiologische Untersuchung \\
\hline fRV & funktioneller rechter Ventrikel \\
\hline $\mathrm{HF}$ & Herzfrequenz \\
\hline $\mathrm{HR}$ & hazard ratio \\
\hline $\mathrm{HZV}$ & Herzzeitvolumen \\
\hline LA & linkes Atrium/linker Vorhof \\
\hline LV & linker Ventrikel \\
\hline $\max . \mathrm{VO}_{2}$ & maximale Sauerstoffaufnahme \\
\hline MRT & Magnetresonanztomographie \\
\hline NYHA & New York Heart Association \\
\hline Qp & Lungenperfusion \\
\hline Qs & Systemperfusion \\
\hline$R / L-V I$ & Totaler Rechts/Links-Volumen-Index \\
\hline RA & rechtes Atrium/rechter Vorhof \\
\hline $\mathrm{RV}$ & rechter Ventrikel \\
\hline $\mathrm{SaO}_{2}$ & transkutane Sauerstoffsättigung \\
\hline SSFP & steady state free precession \\
\hline TAPSE & tricuspid annular plane systolic excursion \\
\hline
\end{tabular}


TEE

$\mathrm{TI}$

TK

$\mathrm{VE} / \mathrm{VCO}_{2}$

WMA transesophageal echocardiography

Trikuspidalklappeninsuffizienz

Trikuspidalklappe

exspiratorischer $\mathrm{CO}_{2}$-Quotient

World Medical Association 


\section{Einleitung}

Diese Dissertation befasst sich mit der Validierung und Evaluierung des Totalen Rechts/Links-Volumen-Index (R/L-VI) (Hösch et al. 2014). Dies ist ein neuer MRTParameter, der von der interdisziplinären Arbeitsgruppe „Kardiale Bildgebung“ der Universitätsmedizin Göttingen im Jahr 2014 erarbeitet und eingeführt wurde. Der R/LVI soll zur Einteilung des Schweregrades der Ebstein'schen Anomalie (EA) genutzt werden und als prognostischer Parameter für die Erkrankung dienen. Im Rahmen dieser Dissertation wird die Aussagekraft und klinische Anwendbarkeit des Index in einer longitudinalen Nachuntersuchung evaluiert und diskutiert.

\subsection{Ebstein Anomalie}

Die EA ist ein sehr seltener angeborener Herzfehler, der definiert wird als Malformation der Trikuspidalklappe (TK) des rechten Herzens. Das morphologische Hauptkriterium ist die Verlagerung der Klappenschlussebene zur Herzspitze hin (Lamers et al. 1995).

Der Versatz beträgt bei der EA mindestens $8 \mathrm{~mm} / \mathrm{m}^{2}$ Körperoberfläche (Edwards 1993). Dadurch entsteht eine Zweiteilung des rechten Ventrikels (Engle et al. 1950), wodurch es zu hämodynamischen und funktionellen Veränderungen des Herzens und zur klinischen Manifestation der Erkrankung kommt.

Der Namensgeber der EA, Dr. Wilhelm Ebstein (1836-1912), beschrieb im Jahre 1866 erstmals diese auffällige Malformation der TK bei einem 19-jährigen Patienten. Bei dem jungen Mann waren eine Zyanose, Dyspnoe, Palpitationen, gestaute Jugularvenen und eine Kardiomegalie aufgefallen (Ebstein 1866; Mann et al. 1979). Nach dem Tod des jungen Patienten führte der Göttinger Arzt und Wissenschaftler eine Autopsie durch und entdeckte ein vergrößertes und fenestriertes vorderes Trikuspidalklappensegel, während das hintere und das septale Segel hypoplastisch erschienen und dem rechten Ventrikel (RV) anhafteten. Weiterhin fielen Wilhelm Ebstein ein ausgedünnter und dilatierter Anteil des RV, ein vergrößertes rechtes Atrium (RA) und ein persistierendes Foramen ovale (PFO) auf. Seine Erkenntnisse veröffentlichte Ebstein 1866 in 
dem Bericht „Ueber einen sehr seltenen Fall von Insufficienz der Valvula tricuspidalis, bedingt durch eine angeborene hochgradige Mißbildung derselben“. Erst Jahre nach Ebsteins Bericht wurde über einen weiteren Fall mit einer ähnlichen Anomalie berichtet (Vacca et al. 1958). 60 Jahre später war von insgesamt 14 weiteren Fällen die Rede. Bis zum Jahre 1957 wurden weltweit insgesamt 108 Fälle der EA verzeichnet (Vacca et al. 1958).

Epidemiologisch tritt die Ebstein'sche Malformation der TK bei ca. 1 bis 5 von 200.000 lebendgeborenen Kindern auf und macht weniger als 1\% aller angeborenen Herzfehler aus (Correa-Villaseñor et al. 1994; Edwards 1993). Die Inzidenz der EA liegt bei 0,30,5\% aller Erkrankten mit angeborenen Herzfehlern (Watson et al. 1974). Die Prävalenz betrug in der von Juli 2006 bis Juni 2007 durchgeführten PAN(Prävalenz angeborener Herzfehler bei Neugeborenen)-Studie 0,4 von 10.000 Lebendgeborenen, das Geschlechterverhältnis war ausgeglichen (Ratio: 0,93) (Lindinger et al. 2010). In allen teilnehmenden Instituten Deutschlands wurden in diesem Zeitraum insgesamt 27 Kinder mit EA geboren.

Da es sich um eine angeborene Krankheit handelt, die einen sehr unterschiedlichen klinischen Verlauf nehmen kann, sind alle Altersgruppen betroffen. Die Gesamtprävalenz angeborener Herzfehler liegt in Deutschland bei insgesamt 1,08\% (Lindinger et al. 2010).

Die Ursachen der EA sind bis heute nicht vollständig bekannt und ungenügend erforscht. Es existieren heterogene genetische Faktoren, die zur Entstehung der EA führen können. In Studien werden genetische, reproduktive und Umweltfaktoren genannt, die als Risikofaktoren für die Entstehung der EA gelten (Correa-Villaseñor et al. 1994). Beispielsweise tritt die Anomalie häufiger bei Zwillingen auf, ebenso bei einer positiven Familienanamnese für die Erkrankung. Bei einer Therapie mit Benzodiazepinen während der Schwangerschaft (Correa-Villaseñor et al. 1994) sowie einer Therapie mit Lithium im ersten Trimenon kann eine EA mit höherer Wahrscheinlichkeit bei dem Nachwuchs auftreten. Bei einer Therapie mit Lithium besteht sogar ein 400-fach erhöhtes Risiko für das ungeborene Kind (Cohen et al. 1994). Eine familiäre EA ist sehr 
selten, die meisten Fälle treten sporadisch auf (Postma et al. 2011). Allerdings sind Mutationen im Sarkomer-Protein-Gen MYHA7 (engl. myosin heavy chain) (Engelen et al. 2013), sowie im kardialen Transkriptionsfaktor NKX 2.5 (Benson et al. 1999) als Ursache für eine EA nachgewiesen worden. Desweiteren sind 10p13-p14 Deletionen und 1p34.3-p36.11 Deletionen bei Patienten mit EA beschrieben worden (Yatsenko et al. 2004; Yang et al. 2004).

Embryologisch entwickeln sich das Myokard und die Atrioventrikular(AV)-Klappen aus derselben Anlage. Es sind zwei Mechanismen bekannt, die für die Verlagerung der TKSchlussebene maßgeblich sind. Zum einen die fehlende Trennung der Klappe von der Ventrikelwand, und zum zweiten eine unvollständige Separation, welche mehrere Wandadhäsionen entstehen lässt (Lamers et al. 1995).

In anatomisch unauffälligen Herzen besteht die TK aus drei Segeln, dem anterioren, dem posterioren und dem septalen Segel (Lamers et al. 1995). Demgegenüber steht die auffällige TK eines Patienten mit EA, bei der meist das septale und/oder das hintere (posteriore) Segel dem Myokard angeheftet und unterschiedlich stark nach apikal verlagert sind. Die Leitlinien der Deutschen Gesellschaft für Pädiatrische Kardiologie (DGPK) geben dies als morphologisches Hauptkriterium der Erkrankung an (DGPK 2014). Der Versatz des posterioren oder septalen Segels richtung Herzspitze beträgt bei der EA mehr als $8 \mathrm{~mm} / \mathrm{m}^{2}$ Körperoberfläche (Edwards 1993). Das vordere Segel setzt demgegenüber unverändert am atrioventrikulären Übergang an. Häufig ist das vordere (anteriore) Segel vergrößert und gefenstert, sodass es den rechtsventrikulären Ausflusstrakt teilweise verlegt und den Blutfluss behindert (Paranon und Acar 2008). Das posteriore und das septale Segel weisen häufig mehrere Adhäsionen auf oder verwachsen gar zu einem einzigen Segel (Zuberbuhler et al. 1984). Durch die Verlagerung der TK-Schlussebene in den Ventrikel wird der RV zweigeteilt. Basal der Klappe wird ein Anteil des RV „atrialisiert“, unterhalb der Klappe befindet sich der funktionelle, ursprüngliche RV (Engle et al. 1950; Lamers et al. 1995). Obwohl der atrialisierte rechte Ventrikel (aRV) funktionell zum rechten Atrium (RA) gehört, kontrahiert er mit dem funktionellen rechten Ventrikel (fRV). Der RV ist bei bis zu 66\% der Patienten mit EA vergrößert. Die Wand des RV ist dadurch häufig ausgedünnt und atrophisch (Ander- 
son et al. 1979). Es besteht häufig zusätzlich eine höhergradige Trikuspidalklappeninsuffizienz (TI) (Attenhofer Jost et al. 2007). Das RA sowie der aRV und der fRV sind abhängig vom Ausmaß der TI vergrößert (Fratz et al. 2013b). Der fRV bildet bei EA Patienten häufig sogar die Herzspitze. Der fRV verändert durch seine Fehlbildung auch die Funktion und die Form des linken Ventrikels (LV). 39\% der Patienten mit EA zeigen zusätzlich Anomalien des linken Herzens. Hierbei sind hauptsächlich die Klappen und/oder das Myokard betroffen (Attenhofer Jost et al. 2005). Der LV weist zusätzlich vor allem im basalen Anteil des Septums häufig eine Dys- und Hypokinesie auf (Goleski et al. 2014). Diese Veränderungen der Herzklappen und Herzkammern führen ebenso zu einer veränderten globalen Herzfunktion.

Laut den Leitlinien der DGPK von 2014 sind die wichtigsten hämodynamischen Veränderungen bei Patienten mit EA die Volumenbelastung und Funktionseinschränkung des RV, sowie ein reduzierter transpulmonaler Nettovorwärtsfluss. Durch diesen wird die Vorlast des LV vermindert und das Herzzeitvolumen (HZV) sinkt. Durch den Versatz und die Anheftung der Segel der TK wird diese insuffizient, während das RA dilatiert (Fratz et al. 2013b). Infolge dessen kommt es zu einer chronischen pulmonalen Minderperfusion. Zudem verursacht die abnorme Anatomie des Herzens eine Veränderung der Mechanokinetik aller Herzkammern, der basale Teil des Ventrikelseptums wird häufig dyskinetisch (Goleski et al. 2014). Hierdurch werden die Kontraktionskraft und die Gesamtfunktion des Herzens stark beeinflusst.

Bei der EA können auch assoziierte Fehlbildungen auftreten. Laut den Leitlinien der DGPK von 2014 ist ein Vorhofseptumdefekt bzw. ein PFO die häufigste mit der EA assoziierte Fehlbildung. Eine interatriale Kommunikation besteht bei $80-94 \%$ aller Patienten mit EA (Danielson et al. 1992; Brickner et al. 2000).

Es kann ein links-rechts-Shunt bestehen oder gar durch einen rechts-links-Shunt eine Zyanose verursacht werden. Durch die Verlagerung des septalen TK-Segels entstehen häufig Unregelmäßigkeiten im fibrösen Herzskelett und im septalen atrioventrikulären Ring (DGPK 2014). Somit entstehen direkte muskuläre atrioventrikuläre Verbindungen und dadurch potenziell auch akzessorische atrioventrikuläre Leitungsbahnen und 
ventrikuläre Präexzitationen. Ein Beispiel hierfür ist das Wolff-Parkinson-WhiteSyndrom, die Prävalenz bei der EA beträgt hierfür bis zu 44\% (Delhaas et al. 2010). Eine Erklärung für das erhöhte Risiko für Vorhofflattern oder Vorhofflimmern ist die Vergrößerung des Vorhofs, welche das Auftreten von atrialen Tachyarrhythmien wahrscheinlicher macht (DGPK 2014). Weitere assoziierte Anomalien sind bikuspide oder atretische Aortenklappen, Pulmonalatresien oder hypoplastische Pulmonalarterien. Ferner können subaortal Stenosen oder Mitralklappenprolapse auftreten. Ventrikuläre Septumdefekte und Pulmonalstenosen sowie Malformationen des LV sind ebenfalls assoziiert (Attenhofer Jost et al. 2007).

Die klinischen Leitsymptome der EA hängen von dem Alter der Patienten, dem Schweregrad, den hämodynamischen Gegebenheiten und eventueller rechts-links-Shunts ab (Giuliani et al. 1979). Häufig fallen Kinder mit morphologisch schwerwiegenden Formen der EA schon früh, teilweise bereits im Neugeborenenalter durch eine zentrale Zyanose auf (Celermajer et al. 1992). Bei Kleinkindern können Symptome einer Rechtsherzinsuffizienz bemerkbar sein, bei Schulkindern imponiert hingegen oft lediglich ein uncharakteristisches systolisches Herzgeräusch bzw. ein fixiert gespaltener zweiter Herzton (Giuliani et al. 1979). Tachyarrhythmien sind das Leitsymptom bei Jugendlichen und Erwachsenen, da tachykarde Herzrhythmusstörungen bei Patienten mit EA im Vergleich zu anderen Herzfehlern deutlich häufiger auftreten (Lindinger et al. 2010). Auch wenn die Patienten subjektiv symptomfrei sind, weisen sie oft objektiv eine eingeschränkte körperliche Belastbarkeit auf (Müller et al. 2011; MacLellanTobert et al. 1997).

Für Patienten mit EA besteht das Risiko für eine paradoxe Embolie falls eine interatriale Verbindung besteht (Celermajer et al. 1994). Als weitere Komplikationen für Patienten mit EA ist der plötzliche Herztod zu nennen, der bei ventrikulären Arrythmien eintreten kann (Celermajer et al. 1992).

Die Schwere bzw. der Ausprägungsgrad der Anomalie ist sehr unterschiedlich. Somit sind auch die Auswirkungen für die Betroffenen teils milde, teils beträchtlich. Die Lebensqualität kann uneingeschränkt sein, wobei viele Patienten unter starken Symptomen der Herzinssuffizienz leiden und sich körperlich als deutlich weniger belastbar 
empfinden als Gesunde. Bei einem starken Ausprägungsgrad kann die EA bereits für Neugeborene letal enden, während Patienten mit einer leichten Form der EA eine normale Lebenserwartung haben können (Makous \& Vander Veer 1966).

Zur Diagnostik der EA stehen mehrere apparative Untersuchungsmethoden zur Vefügung. Als momentan wichtigstes apparatives Mittel zur Diagnostik der EA gilt die Echokardiographie. Durch eine echokardiographische Untersuchung kann sowohl die Anatomie des Herzens als auch die Hämodynamik genau dargestellt werden. Auch die Mobilität der TK-Segel und die TI, sowie die Größe und Funktion des RV können von erfahrenen Untersuchern echokardiographisch beurteilt werden. Somit gilt sie bis heute laut DGPK als primäre Methode zur Diagnosestellung der EA (Attenhofer Jost et al. 2012; Bharucha et al. 2010; Ahmed et al. 2003).

Zusätzlich steht laut DGPK die Objektivierung der körperlichen Belastbarkeit im Vordergrund. Hierzu dienen die Messung der Sauerstoffsättigung mittels Pulsoxymetrie sowie die Durchführung einer spiroergometrischen Untersuchung. Letzere dient der Verlaufsbeobachtung und ist Bestandteil der Therapieplanung (DGPK 2014). Als weitere bildgebende Diagnostik dient eine Röntgen-Thorax-Aufnahme, die allerdings der aktuellen DGPK-Leitlinie zufolge für die Diagnosestellung entbehrlich ist. Sichtbar ist im Röntgen-Thorax häufig eine vergrößerte Herzsilhouette durch den großen RA. Im Elektrokardiogramm (EKG) und im Langzeit-Elektrokardiogramm (LZ-EKG) zeigen sich bei der Mehrzahl der Patienten Auffälligkeiten wie eine verbreiterte P-Welle, eine verlängerte PQ-Zeit oder Präexzitationen. Auch inkomplette oder komplette Rechtsschenkelblöcke sind häufig (DGPK 2014). AV-Blöcke ersten Grades treten bei 42\% der Patienten mit EA auf (Giuliani et al. 1979; Ho et al. 2000). Da supraventrikuläre Tachykardien bei Patienten mit EA keine Rarität sind, sollte die Ableitung eines 24-Stunden-EKGs bei Durchführung der Primärdiagnostik erfolgen und bei Bedarf bei Verlaufskontrollen wiederholt werden. Eine Herzkatheteruntersuchung ist zur Sicherung der Diagnose nicht indiziert. Allerdings wird beim Vorhandensein von akzessorischen Leitungsbahnen von der DGPK eine elektrophysiologische Untersuchung (EPU) und gegebenenfalls eine Ablationsbehandlung empfohlen (DGPK 2014). 
Laut den aktuellen Leitlinien des DGPK ist bis heute eine Magnetresonanztherapie (MRT) oder Computertomographie(CT)-Untersuchung nicht zwingend indiziert, kann aber für die Indikationsstellung einer Therapiemaßnahme durchaus von Nutzen sein. Zur Quantifizierung der rechts- und linksventrikulären Funktion ist laut DGPK aktuell die kardiale MRT(cMRT)-Untersuchung am besten geeignet (DGPK 2014).

Die Therapiemöglichkeiten der EA sind abhängig vom Ausprägungsgrad der Erkankung und dem klinischen Zustand des Patienten. Daher ist die wichtigste medizinische Maßnahme die regelmäßige Überwachung und Reevaluierung des klinischen Zustandes des Erkrankten durch spezialisierte Kardiologen. Diese Vorsorge wird in Deutschland von der DGPK ein- bis zweimal jährlich empfohlen und beinhaltet neben der körperlichen Untersuchung ein EKG und die Echokardiographie. Auch eine Spiroergometrie, ein LZEKG und weitere apparative kardiale Funktionsuntersuchungen wie die cMRT können zur Einschätzung des klinischen Zustands der Patienten hilfreich sein (DGPK 2014). In den 2014 erschienenen Leitlinien der DGPK zur EA werden verschiedene Therapiemöglichkeiten genannt. Neugeborene mit einer stark ausgeprägten Form der EA und hochgradiger TI sowie zentraler Zyanose haben eine schlechte Prognose (Celermajer et al. 1994; Knott-Craig et al. 2002). Ein Team aus Kinderkardiologen, Intensivmedizinern und Kinderherzchirurgen sollte in solchen Fällen über die passende Behandlung entscheiden (DGPK 2014). Alle intensivmedizinisch möglichen Maßnahmen sollten vor der Durchführung von palliativen Eingriffen wie der Anlage eines aorto-pulmonalen Shunts oder Ductusstenting ergriffen werden. Durch die symptomatische medikamentöse Behandlung mit Prostaglandin E bei Neugeborenen mit Ductusabhängigkeit kann über Wochen eine neonatale Operation vermieden werden.

Bei frühzeitig auftretenden supraventrikulären Tachykardien liegen häufig mehrere akzessorische Leitungsbahnen vor, die in $80 \%$ erfolgreich abladiert werden können (DGPK 2014). Im Vergleich zu Patienten mit strukturell normalen Herzen, bei denen die Erfolsrate bei $95 \%$ liegt, gelingt die Katheterablation bei Patienten mit EA allerdings seltener und das Risiko für das Neuauftreten von supraventrikulären Tachykardien ist höher (Hebe et al. 2000; Reich et al. 1998; Cappato et al. 1996). 
Es stehen eine Reihe chirurgischer Therapiemaßnahmen zur Verfügung. Korrigierende rekonstruktive Verfahren können klappenerhaltend durchgeführt werden oder durch einen mechanischen bzw. biologischen Klappenersatz erfolgen. Für die rekonstruktiven Operationsverfahren existieren verschiedene Konzepte wie z.B. die Plikation bestimmter Anteile des RV oder die Veränderung der Klappenaufhängung durch das Rotieren und eine nachfolgende Neuinsertion der Segel im anatomischen Klappenring (Danielson et al. 1992; Carpentier et al. 1988). Oft müssen allerdings nachfolgend weitere Operationen durchgeführt werden.

Palliative Operationsverfahren sind beispielsweise die Anlage eines aorto-pulmonalen Shunts und der zusätzliche Verschluss des Trikuspidalklappenostiums mit einem fenestrierten Patch, die sogenannte „Starnes-Operation“ (Reemtsen et al. 2007). Anschließend wird eine kavo-pulmonale Anastomose geschaffen, sodass eine univentrikuläre Kreislaufsituation entsteht.

Die Indikationen für den Verschluss eines Vorhofseptumdefekts, einen Trikuspidalklappenersatz oder eine operative TK-Rekonstruktion sind bisher leider nur durch wenige prospektive Daten untermauert. Im Jahre 1962 wurde erstmals der erfolgreiche TK-Ersatz bei einem Patienten mit EA beschrieben. Damals lag die Mortalität bei 54\% (Watson et al. 1974).

1988 beschrieben Carpentier et al. eine Operation, durch die das anteriore Segel der TK mobilisiert werden konnte. Diese Operation wurde in einer Studie von 2003 an 191 Patienten durchgeführt, es verstarben $9 \%$ frühzeitig und die Langzeitüberlebensrate lag nach 20 Jahren bei $82 \pm 5 \%$. (Chauvaud et al. 2003). In einer weiteren Studie aus dem Jahr 1998 wurden 294 Patienten beobachtet, die entweder eine klappenerhaltende Rekonstruktion oder einen Klappenersatz mit Bioprothese bzw. mechanischer Klappe erhielten. Es wurde kontrolliert, ob eine Reoperation notwendig wurde. Hierbei zeigte sich kein signifikanter Unterschied zwischen den zwei Gruppen (Kiziltan et al. 1998).

Bis heute konnte nicht ausreichend nachgewiesen werden, ob die Rekonstruktion oder der Ersatz der TK bessere Langzeitresultate erzielt. Noch weiß man, ob Bioprothesen oder mechanische Prothesen als TK-Ersatz zu präferieren sind (Attenhofer Jost et al. 2007). 
Derzeit ist die sogenannte Cone-Operation nach da Silva in vielen Zentren Deutschlands das favorisierte Operationsverfahren bei Patienten mit EA. Hierbei werden das anteriore und das posteriore TK-Segel von ihreren Anheftungen an den RV gelöst. Die nun freien Enden werden rotiert und zusammengefasst, sodass ein Kegel (engl. „cone“) entsteht, dessen Scheitelpunkt an der Spitze des RV liegt und dessen Basis am wirklichen TK-Anulus angenäht wird (da Silva et al. 2012).

Erste Untersuchungen zu dem früh- bis mittelfristigen Outcome bei 40 Patienten mit EA, die nach dem Cone-Rekonstruktionsverfahren operiert wurden, ergaben bei einer vierjährigen Folgestudie zufriedenstellende Ergebnisse. Zwei Todesfälle stehen einer generellen Verbesserung der NYHA-Klasse und der TI gegenüber (da Silva et al. 2007). Eine Studie mit weiteren 100 Patienten ergab eine Mortalitätsrate von 3\%, ein generell gutes klinisches Outcome und keine Notwendigkeit für einen späteren TK-Ersatz (da Silva und da Silva 2012). Auch die Größe des RV reduziert sich nach Durchführung der Cone-Operation langfristig, was durch eine Studie mit 20 Patienten im Jahr 2015 von Lange et al. nachgewiesen wurde (Lange et al. 2015).

Dennoch ist die Entscheidung für oder gegen eine Operation oft schwer zu fällen und sollte deshalb von einem Expertenteam getroffen werden (DGPK 2014). Hierfür könnte eine einheitliche Klassifikation der EA von großem Nutzen sein und somit der Entscheidungsfindung über die Art und den passenden Zeitpunkt der Therapie und eine Evaluation des Outcomes erleichtern.

Bis heute existiert allerdings keine einheitliche Einteilung des Schweregrads der EA. Meist erfolgt primär eine Einteilung mittels Echokardiographie. Anhand dieser kann der Herzfehler in anatomisch mild, moderat oder schwer eingeteilt werden. In diese Beurteilungen gehen der TK-Versatz und die Größe des RV mit ein. Es ist zwar eine wenig präzise aber simple Einteilung (Attenhofer Jost et al. 2007). Generell sind in der Klinik allerdings mehrere unterschiedliche Klassifizierungsweisen bekannt. Celermajer et al. etablierten 1992 ein echokardiographisches Scoring System von 1 bis 4 bei Neugeborenen. Danach ist eine Unterscheidung in vier Schweregrade möglich. In der Darstellung des Vier-Kammer-Blicks (4CV) in der Enddiastole (ED) wird der Quotient der Summe der Flächen von RA und aRV und der Summe der Flächen von fRV, LV und dem 
linken Atrium (LA) errechnet. Beträgt der Quotient dieser Flächen $(\mathrm{RA}+\mathrm{aRV}) /(\mathrm{fRV}+\mathrm{LA}+\mathrm{LV})<0,5$ so wird die Ausprägung der EA dem Schweregrad I zugeordnet. Liegt der Quotient zwischen 0,5 und 0,99 liegt der Schweregrad II vor, zwischen 1,0 und 1,49 der Schweregrad III und > 1,5 der Schweregrad IV (siehe Abbildung $1)$.

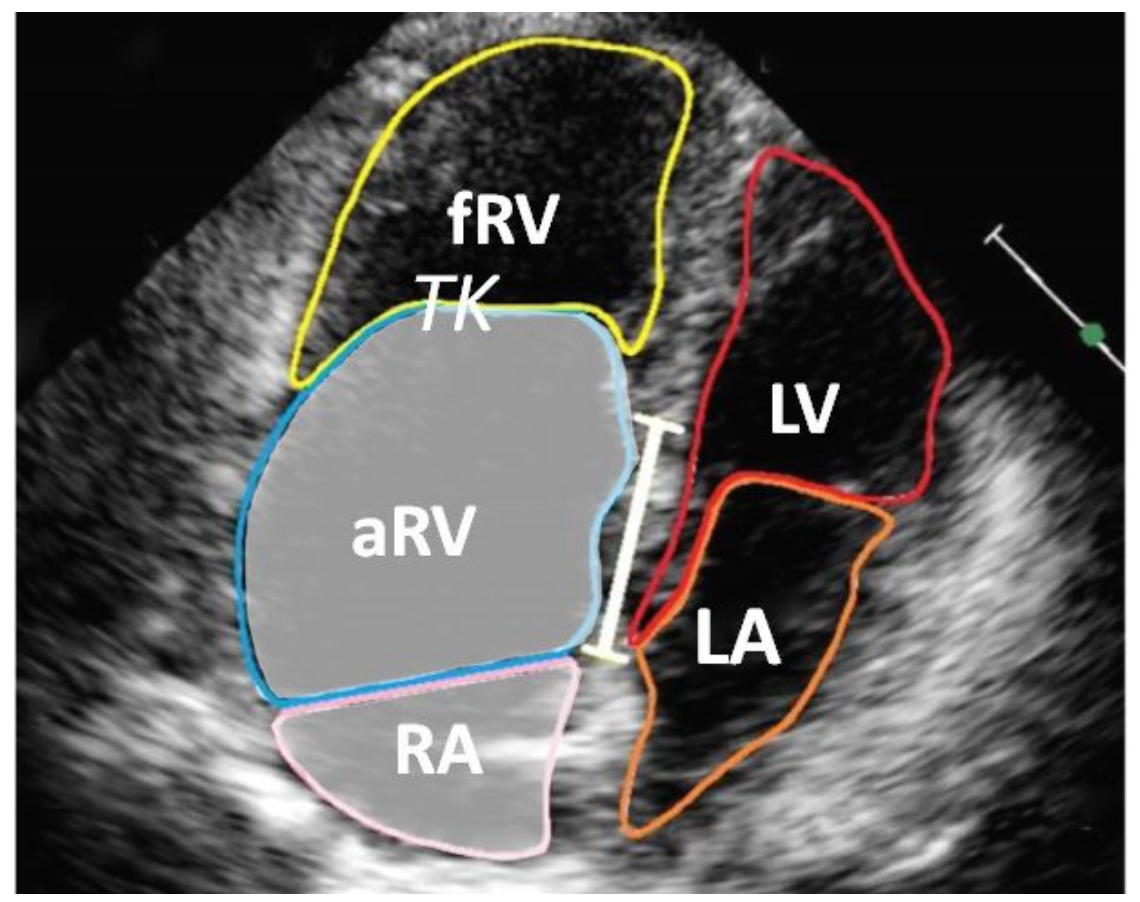

Abbildung 1: Echokardiographische Einteilung der Ebstein Anomalie gemäß Celermajer im enddiastolischen Vier-Kammer-Blick (Celermajer et al. 1992; Hösch 2015; mit freundlicher Genehmigung der Autoren).

$\mathrm{aRV}=$ atrialisierter rechter Ventrikel; $\mathrm{fRV}=$ funktioneller rechter Ventrikel; $\mathrm{LA}=$ linkes Atrium; $\mathrm{LV}=$ linker Ventrikel; RA = rechtes Atrium; TK = Trikuspidalklappe.

Carpentier et al. schlugen 1988 eine Einteilung in vier Typen (A, B, C und D) vor. Die Schwere wurde aus chirurgischer Sicht anhand der Klappenmorphologie und der Morphologie des rechten Herzens von einem leichtgradigen Typ A bis zu einem schwergradigen Typ D eingeteilt (siehe Abbildung 2). 

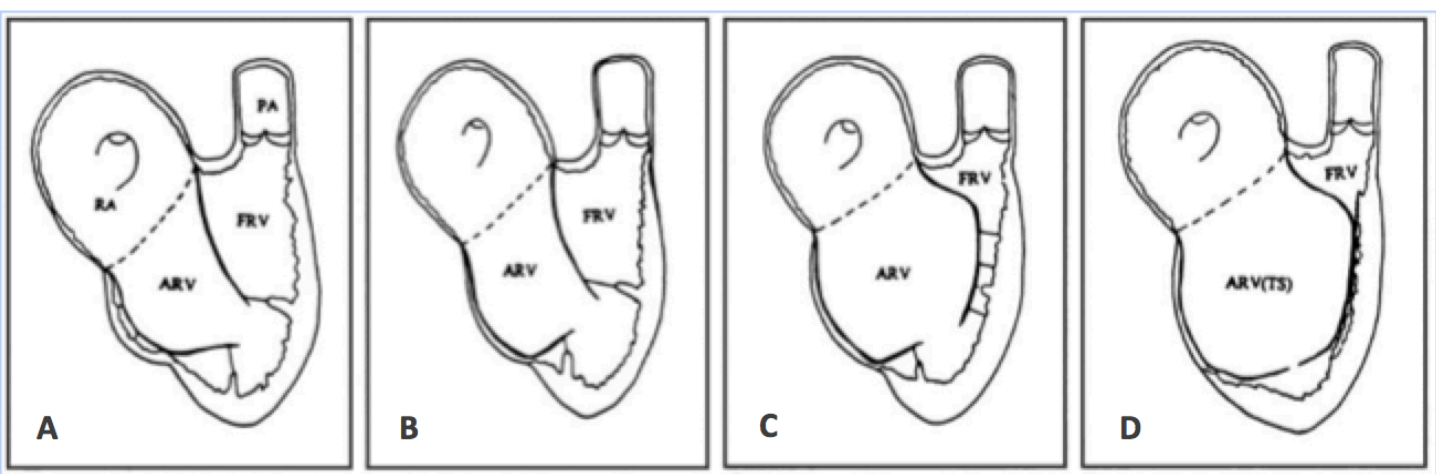

Abbildung 2: Anatomische Einteilung der Ebstein Anomalie gemäß Carpentier et al. (1988; mit freundlicher Genehmigung des Elsevier-Verlags).

ARV = atrialisierter rechter Vetrikel; FRV = funktioneller rechter Ventrikel; PA = Pulmonalarterie; $R A=$ rechtes Atrium.

Bei Typ A ist das Volumen des RV adäquat, der kontraktile aRV ist klein und hat ein bewegliches vorderes Klappensegel. Typ B zeigt einen großen atrialisierten Anteil des RV, das vordere Segel der TK kann sich hier ebenfalls frei bewegen. Bei Typ C ist das vordere Segel der TK nur bedingt beweglich und verursacht teilweise eine Obstruktion des rechtsventrikulären Ausflusstraktes. Bei Typ D ist der RV fast vollständig atrialisiert, sodass nur ein kleiner Anteil des fRV übrig bleibt. Die Segel bilden bei diesem Typen einen kontinuierlichen, am dilatierten fRV anhaftenden Sack (Carpentier et al. 1988). Laut den Leitlinien der DGPK von 2014 ist eine Klassifikation entsprechend der Anatomie sinnvoll, da die EA morphologisch eine erhebliche Varianz aufweist (DGPK 2014). Somit richtet sich momentan die Einteilung meist nach Alain Carpentier.

Im klinischen Alltag werden jedoch weder die Einteilung nach Carpentier noch die Einteilung nach Celermajer häufig angewendet. Meist wird lediglich im echokardiographischen enddiastolischen 4CV die Länge des TK-Versatzes vermessen und zur Einteilung genutzt (Hösch 2015).

Die Arbeitsgruppe „Kardiale Bildgebung“ der Universitätsmedizin Göttingen (UMG) begann 2013 mit der Entwicklung des Totalen Rechts/Links-Volumen-Index, der zur Einteilung des Schweregrades und zur Klassifikation der EA dienen soll.

Er wird anhand von CMRT-Bilddaten berechnet und gibt die Relation der Volumina der Kammern des rechten Herzens zu den Volumina des linken Herzens wieder. 
Der $\mathrm{R} / \mathrm{L}-\mathrm{VI}$ ergibt sich aus den enddiastolischen Volumina der rechten Herzkammern geteilt durch die Volumina der linken Herzkammern. Die Formel zur Errechnung des Totalen R/L-VI lautet: $R / L-V I=(R A+a R V+f R V) /(L A+L V)$ (siehe Abbildung 3).

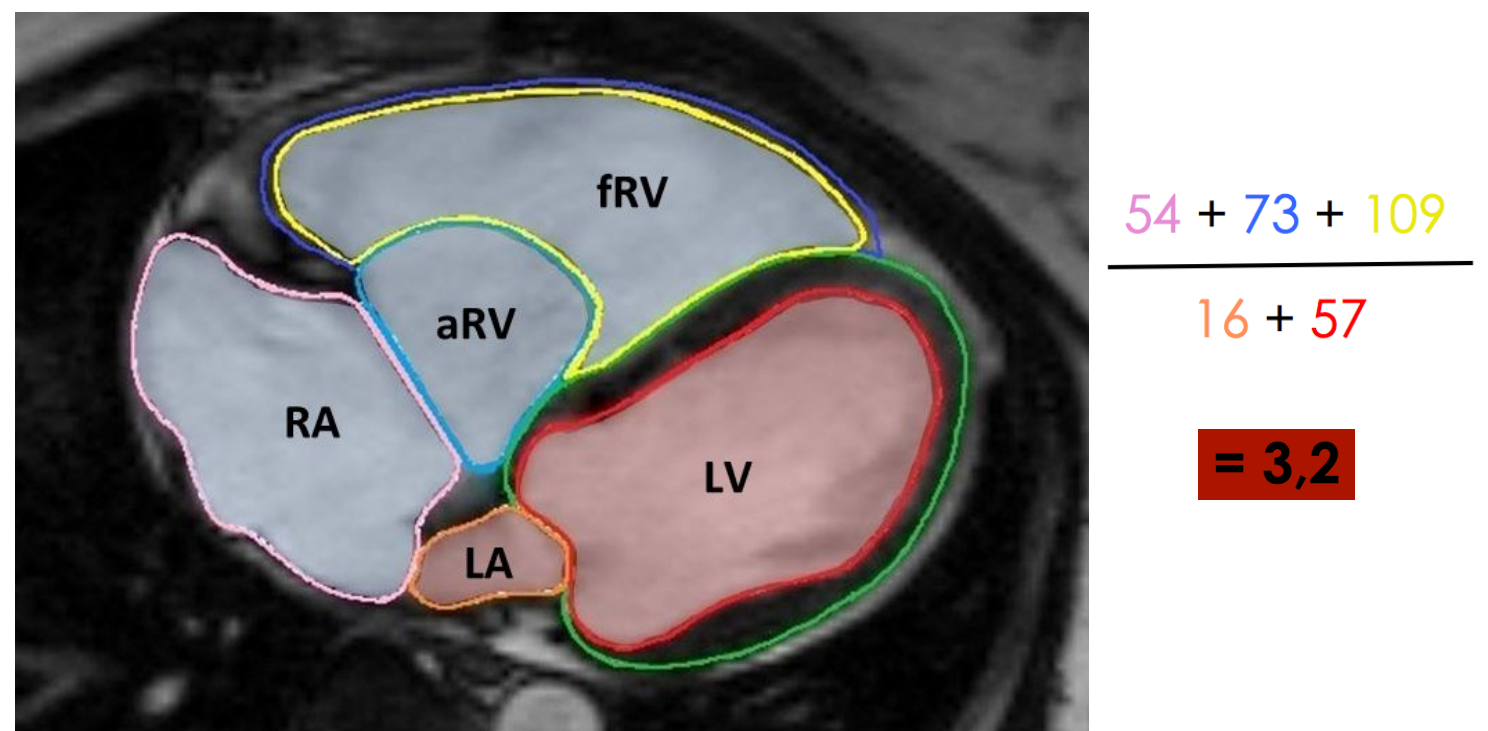

Abbildung 3: Illustration der Berechnung des Totalen Rechts/Links-Volumen-Index in axialer Bildebene mit Formel (Hösch et al. 2014; mit freundlicher Genehmigung der Autoren und des Wolters Kluwer Health, Inc. Verlags).

$\mathrm{aRV}=$ atrialisierter rechter Ventrikel; fRV = funktioneller rechter Ventrikel; $L A=$ linkes Atrium; $\mathrm{LV}=$ linker Ventrikel; RA = rechtes Atrium.

Die Referenzwerte des R/L-VI für gesunde Probanden betragen 1,1 0,1 (Grenzwerte: 1,0 - 1,2) (Hösch 2015). Anhand von vier Studien an gesunden Probanden wurden diese Referenzwerte für den neuen $\mathrm{R} / \mathrm{L}-\mathrm{VI}$ aus der aktuellen Literatur zusammengestellt (Hudsmith et al. 2005; Sievers et al. 2007; Sarikouch et al. 2010; Sarikouch et al. 2011). Diese wurden für Erwachsene und für Kinder von 8 bis 18 Jahren sowie geschlechtsspezifisch getrennt berechnet (Hösch 2015). Bei den untersuchten Patienten mit EA betrug der R/L-VI in dieser Studie im Mittel 2,6 $\pm 1,3$.

Zudem zeigten die Studien eine gute Korrelation des R/L-VI mit Herzinsuffizienz- und Belastungsparametern.

Der R/L-VI wurde im Jahr 2014 erstmals publiziert und ermöglicht eine einfache Einteilung des Schweregrades der EA aus der Bildgebung, ohne dass die komplexe Anatomie der TK die Segmentierung der Herzhöhlen erschwert (Hösch et al. 2014). 


\subsection{Kardiale Magnetresonanztomographie}

Die Magnetresonanztomographie ist ein Bildgebungsverfahren, das durch starke Magnetfelder und Radiowellen Bilder von Geweben und Organen des menschlichen Körpers erzeugt. Das physikalische Prinzip, auf welchem die MRT beruht, basiert auf der Eigenbewegung von Atomkernen in magnetischen Feldern. Positiv geladene Teilchen, sogenannte Protonen, sind in allen Molekülen des Körpers enthalten und besitzen einen Eigendrehimpuls, welcher Kernspin gennant wird. Die positive Ladung der Protonen dreht sich kontinuierlich um die eigene Achse. Dadurch induziert sie ein eigenes magnetisches Dipolmoment (Hünerbein 2011). Normalerweise liegen diese magnetischen Felder ohne Ordnung vor und es kommt zur gegenseitigen Kompensation. Wenn ein starkes einheitliches Magnetfeld von extern auf sie einwirkt, entsteht eine parallele oder antiparallele Ausrichtung der magnetischen Dipole entlang der Feldlinien des Magnetens (Rodgers und Robson 2011).

Im nächsten Schritt werden die Protonen aus dieser Ausrichtung wieder ausgelenkt. Diesen Auslenkungswinkel nennt man Flipwinkel. Die Auslenkung erfolgt durch das Aussenden von Radiofrequenzimpulsen, welche exakt der Resonanzfrequenz der Protonen entsprechen. Durch das Auftreffen der Radiofrequenzimpulse auf die Protonen verändert sich deren Kernspin. Wenn die Impulse abgeschaltet werden, finden die Protonen wieder in den Ausgangszustand zurück. Hierdurch entsteht ein Resonanzsignal. Dieses Resonanzsignal ist messbar und kann dazu genutzt werden ein MRT-Bild zu erzeugen (Hünerbein 2011).

Mehrere spezifische zeitliche Intervalle sind maßgeblich für die Errechnung des Endbildes. Hierunter zum einen die Echozeit (TE), welche die Zeit von der Anregung bis zur Messung des Resonanzsignal angibt, zum anderen die Repetitionszeit (TR), welche das zeitliche Intervall zwischen zwei Anregnungsimpulsen misst (Lackner et al. 1987).

Mit welcher Geschwindigkeit die Abgabe der Energie erfolgt und die Protonen wieder in ihren Ausgangszustand zurück gelangen können, wird jeweils von der chemischen Umgebung der Protonen bestimmt. Die T1- und T2-Zeiten sind die wichtigsten Messgrößen dieses Relaxationsvorganges. Durch sie werden die verschiedenen Eigenschaften der MRT-Gewebe definiert. Dadurch bilden sie die Grundlage für die MRTBildgebung (Hünerbein 2011). 
Der Magnetresonanztomograph besteht aus verschiedenen Bauteilen. Für die Bildgewinnung maßgeblich ist ein supraleitender Magnet, welcher ein ausreichend starkes und homogenes Magnetfeld erzeugt. Mobile Hochfrequenzspulen, die als Antennen zum Senden und Empfangen der Signale dienen, werden Oberflächenspulen genannt und sollten dem Untersuchungsobjekt möglichst dicht anliegen. Das Signal der Protonen wird von diesen Spulen registriert und an ein Computersystem weitergeleitet. Das Computersystem errechnet im letzten Schritt das Endbild (Lackner et al. 1987).

Durch die heutige MRT-Technik ist es möglich hochauflösende Bilder zu erzeugen und die verschiedenen Organe und Weichteilgewebe des Körpers darzustellen, weswegen die MRT in allen Fachbereichen der Medizin eingesetzt wird.

Auch in der Kardiologie sind durch die MRT massive Fortschritte verzeichnet worden. Bereits in den 1980er Jahren wurden die ersten Ansätze der kardialen MRT-Diagnostik entwickelt. Goldman et al. proklamierten schon damals die MRT als zukunftsfähige Methode für die Darstellung des Herzens (Goldman et al. 1980). Hawkes et al. gelang es 1981 als eine der ersten Arbeitsgruppen das Herz mittels MRT darzustellen. Die kardiale MRT ist heutzutage bei den unterschiedlichsten klinischen Fragestellungen die primär eingesetzte diagnostische Methode, da sie im Gegensatz zu anderen bildgebenden Verfahren keine Strahlenbelastung aufweist und eine der höchsten räumlichen und zeitlichen Auflösungen hat (Saeed et al. 2015).

Eine häufig eingesetzte Gradientenechosequenz in der kardialen MRT heißt steady state free precession (SSFP). Der Flipwinkel beträgt bei dieser Sequenz $<90^{\circ}$. Hierdurch verkürzt sich die TE deutlich und das Signal wird insgesamt verstärkt. Fettgewebe und Flüssigkeiten werden in der SSFP hell dargestellt. Auf den Bildern werden die Grenzen zwischen Blut und Endokard sowie zwischen Epikard und Fettgewebe deutlich sichtbar durch den unterschiedlichen Protonengehalt des Wasser- und Fettgewebes. Aufgrund der starken Kontrastierung zwischen Myokard und Blut werden SSFP-Echosequenzen in der kardialen MRT-Bildgebung heute häufig verwendet (Rodgers und Robson 2011). Die MRT-Aufnahmen in der SSFP erfolgen als CINE-Aufnahme und entsprechen einer kurzen Filmschleife. Das Herz bewegt sich kontinuierlich. Um trotz der ständigen Bewugung eine gute Aufnahme des Herzens zu erzielen, darf die Bildgewinnung nur Bruchteile von Sekunden dauern. Indem alle notwenidgen Daten für ein singuläres Bild 
über mehrere Herzschläge gewonnen werden, wird dieses Problem umgangen. Es entsteht eine Bildserie, die wie ein kleiner Kinofilm abgespielt werden kann und alle Herzphasen zeigt. Diese Sequenz nennt man CINE-Sequenz. Während der MRTUntersuchung wird bei jedem Patienten gleichzeitig zur Bildgewinnung ein EKG aufgezeichnet um die Bilddaten nachfolgend der korrekten Herzphase zuzuordnen. Hierdurch kann die MRT-Sequenz dem EKG angeglichen und die Datengewinnung mit dem Herzschlag in Übereinstimmung gebracht werden (Ridgway 2010). Die Bildgewinnung erfolgt bis zu dem Zeitpunkt an dem genügend Daten vorhanden sind, um einen gesamten Herzzyklus zu berechnen. Die Sortierung der Daten erfolgt widerum anhand des aufgezeichneten EKGs (Ridgway 2010). Durch retrospektives Gating können in der CINE-Sequenz alle Herzphasen dargestellt werden. Die gesamte Funktion des Herzens kann hierdurch analysiert werden. CINE-Aufnahmen gelingen allerdings nur während Atempausen. Für eine CINE-Aufnahme werden zwischen 10 und 20 Herzschläge benötigt (Bluemke et al. 1997; Kellman et al. 2009). Hierfür muss der Proband für insgesamt ca. 15 Sekunden die Luft anhalten, was für Alte und Patienten mit Luftnot problematisch sein kann. Dennoch gilt die Kombination aus CINE-Aufnahmen und SSFP-Sequenz als beste Methode für die Analyse der Funktion des Herzens (Kramer et al. 2013).

Um die Morphologie der Herzkammern vollständig erfassen zu können, werden unterschiedliche Schnittebenen zur Bildgewinnung genutzt. Für diese Studie sind folgende Schnittebenen besonders von Bedeutung: Der 4CV, ein Langachsenschnitt, der vertikal auf der Herzspitze steht und die Mitralklappe und TK mittig schneidet. Im 4CV sind beide Ventrikel und Vorhöfe zu sehen. Zusätzlich der Kurzachsen (SA)-Stapel, bei dem der Schnitt orthogonal zum 4CV und parallel zur Ebene der AV-Klappen verläuft, welcher beide Ventrikel und Vorhöfe zeigt und das gesamte Ventrikel- und Vorhofmyokard abbildet. Werden alle SA-Schichten zusammengenommen, ergibt dies eine Darstellung des Herzens in 3D (Moon et al. 2002).

Mittels cMRT können so konkrete Aussagen über die Herzgröße, die Herzfunktion, die Volumina, die Muskelmasse, die Klappenmorphologie, Fibrosen und eine eventuelle Dyssynchronie gemacht werden (Gutberlet et al. 2000; Attenhofer Jost et al. 2012; Negoi et al. 2013). Es ist so eine umfassende segmentale anatomische Beurteilung des 
Herzens möglich (Attenhofer Jost et al. 2012). Laut zahlreichen Studien gilt die cMRT heute aus diesem Grund als Referenzstandard für die Maße und die Funktionsanalyse des LV und RV (Dewey et al. 2006; Zhou et al. 2015). Die MRT hat sich als beste radiologische Methode zur Darstellung und Beurteilung des RV erwiesen, welcher eine zentrale Rolle bei Patienten mit angeborenen Herzfehlern spielt (Fratz et al. 2013a), da sich die Anatomie wie auch die Blutflüsse außerordentlich genau beurteilen lassen. Dies ist zur Verlaufskontrolle und OP-Indikation von enormer Wichtigkeit. Darum wird die MRT heute in der Diagnostik vieler Herzerkrankungen, wie auch bei der EA, angewandt. Die MRT kann somit wichtige Daten zur Beurteilung der Notwendigkeit von therapeutischen Interventionen liefern (DGPK 2014).

\subsection{Studienziel}

Bis heute existiert keine verlässliche bildgebungsbasierte Schweregradeinteilung der EA. Die Einteilung mittels Echokardiographie nach Celermajer et al. (1992) ist für Neugeborene und Säuglinge hilfreich, hat sich jedoch in der klinischen Verlaufsbeurteilung bei älteren Patienten mit EA nicht durchgesetzt. Es ist weiterhin eine Herausforderung für Kliniker den Schweregrad der EA zu ermitteln und das klinische Procedere dementsprechend zu planen. Im Jahr 2014 konnte die Arbeitsgruppe der Pädiatrischen Kardiologie in Kooperation mit der Abteilung für Diagnostische und Interventionelle Radiologie der Universitätsmedizin Göttingen (UMG) zu diesem Zweck einen neuartigen Bildgebungsindex für den Schweregrad der EA definieren: den Totalen R/L-VI (Hösch et al. 2014). Dieser korrelierte in den ersten Untersuchungen stark mit etablierten klinischen Herzinsuffizienzmarkern und zeigte sich als möglicher Marker für die Schweregradeinteilung.

Ziel der hier vorliegenden Studie ist es, den im Jahr 2014 erstmalig beschriebenen Index hinsichtlich seiner Eignung als sogenannter Bildgebungsbiomarker (,imaging biomarker") zu evaluieren und dessen Reproduzierbarkeit sowie prognostische Aussagekraft zu validieren. Veränderungen der Morphologie und Funktion des Herzens sowie des klinischen Zustandes der Patienten sollten frühzeitig erkannt und erfasst werden. Anhand der longitudinalen Erfassung von klinischen Untersuchungen und cMRT-Daten 
wurde überprüft, ob Veränderungen des klinischen Zustandes mit Veränderungen des $\mathrm{R} / \mathrm{L}-\mathrm{VI}$ einhergehen oder besonders die Patienten mit einem hohen Index von klinischer Verschlechterung betroffen sind. Die Reproduzierbarkeit des Index wurde anhand von Inter- und Intraobserver-Untersuchungen getestet und dessen Anwendbarkeit in der Praxis wird diskutiert.

Folgende Hypothesen sollten im Rahmen der Folgeuntersuchung überprüft werden:

Ist es im Verlauf von drei Jahren zu einer signifikanten Veränderung der MRTMessungen, des R/L-VI oder des klinischen Zustandes der untersuchten Patienten mit EA gekommen? Schlagen sich Veränderungen des klinischen Zustandes der Patienten objektiviert anhand von Herzinsuffizienzparametern und Markern der Leistungsfähigkeit in einer Veränderung des Index nieder? Kann der R/L-VI als bildgebungsbasierter Parameter für die Schweregradeinteilung der EA verwendet werden? Tritt eine Veränderung des klinischen Zustandes insbesondere bei Patienten mit einem hohen R/L-VI von > 2,0 auf? Kann der der R/L-VI zur Risikoberechnung für kardiale Ereignisse und als Vorhersageparameter für das klinische Outcome dienen? Kann der R/L-VI zur Verlaufskontrolle und als Entscheidungshilfe für Therapiemaßnahmen im klinischen Alltag eingesetzt werden? Ist der R/L-VI verlässlich reproduzierbar?

Wenn sich der R/L-VI als valider Parameter zur Klassifikation der EA und als Vorhersageparameter des klinischen Verlaufs der Erkrankung herauskristallisiert, kann das klinische Management und die Behandlung von Patienten mit EA in Zukunft vereinheitlicht und vermutlich verbessert werden. 


\section{Patienten und Methoden}

\subsection{Studiendesign}

Es handelt sich bei der vorliegenden Arbeit um die erste Folgestudie einer prospektivklinischen Studie, mit deren Durchführung im Januar 2013 an der UMG begonnen wurde. Alle damaligen Patienten mit EA wurden drei Jahre nach den ersten Untersuchungen erneut eingeladen und über einen Zeitraum von insgesamt fünf Monaten $(12 / 2015-04 / 2016)$ in der Klinik für Intensivmedizin und Kinderkardiologie der UMG untersucht. Die Ethikkommission genehmigte erneut das Studienprotokoll, welches 2013 für die Durchführung des ersten Teils der Studie erstellt wurde. Das Protokoll stimmt mit den Grundsätzen der Ethik nach der Deklaration von Helsinki über die medizinische Forschung am Menschen überein (WMA 2013).

Die Informationen für Patienten sowie die Einverständniserklärungen wurden jeweils für Kinder und Eltern sowie für Erwachsene in einer patientenverständlichen Formulierung erstellt und für unterschiedliche Altersgruppen angepasst ausgehändigt.

Die aktuelle Studie entspricht den Grundlagen des ersten Teils der Studie von 2013 (Hösch et al. 2014) und wurde anhand der damals erstellten Kriterien und Untersuchungsprotokolle durchgeführt. Die Folgestudie ist somit in der Methodik identisch zu der ersten Durchführung der Studie, wodurch die Vergleichbarkeit gewährleistet werden kann.

\subsection{Patientenkollektiv}

Es wurden 25 Patienten mit EA drei Jahre nach den ersten Untersuchungen für die vorliegende Folgestudie erneut kontaktiert und zu den Nachfolgeuntersuchungen eingeladen. Die folgenden Ein- und Ausschlusskriterien wurden für die Studie definiert: Im Jahr 2013 wurden einwilligungsfähige Kinder, Jugendliche und Erwachsene mit EA ab einem Alter von mindestens zehn Jahren in die Studie aufgenommen. Dementsprechend waren die Patienten bei den Folgeuntersuchungen drei Jahre älter, nun also mindestens 13 Jahre alt. 
Nicht in die Studie aufgenommen wurden Patienten, bei denen Korrekturoperationen der EA durchgeführt worden waren (ausgenommen Atriumseptumdefekt-Verschlüsse), die an komplexen assoziierten angeborenen Herzfehlern litten, diejenigen welche nicht-MRT-taugliche Metalle implantiert bekommen hatten, eine mangelnde Compliance zeigten oder an anderen Studien teilnahmen. Weitere medizinische Gründe wie Herzrhythmusstörungen, Niereninsuffizienz oder Schwangerschaft waren ebenfalls Ausschlusskriterien.

Es erklärten sich 25 Patienten im Jahr 2016 bereit an der Studie teilzunehmen und erfüllten die Einschlusskriterien. Sie gaben nach einer schriftlichen und mündlichen Aufklärung über die Studie ihre schriftliche Einverständniserklärung zur Teilnahme und wurden dem Studienprotokoll von 2013 folgend untersucht. Von den ursprünglich 25 Patienten wurde von zwei Personen die erneute Teilnahme an der Studie abgelehnt, ein Patient war nicht mehr kontaktierbar und ein Patient war leider im Zeitraum von 2013 bis 2016 verstorben. Ein weiterer Patient litt unter Klaustrophobie und musste aus diesem Grund die MRT-Untersuchung abbrechen. Hinzu kamen zwei neue Patienten, deren Daten für die zukünftigen Folgeuntersuchungen genutzt werden können.

\subsection{Klinische Untersuchungen}

Die Untersuchungen fanden innerhalb eines Tages im Rahmen der jährlichen klinischen Routinekontrollen statt, die nach den Leitlinien der Deutschen und Europäischen Gesellschaften für Kardiologie und für Pädiatrische Kardiologie empfohlen werden. Beinhaltet war jeweils die Erhebung der ausführlichen Krankengeschichte, eine umfängliche körperliche Untersuchung, eine Blutentnahme für laborchemische Untersuchungen, ein Standard-Ruhe-EKG (12-Kanal), ein Langzeit-EKG mit Messung über 24 Stunden, eine Spiroergometrie, eine Echokardiographie und eine cMRT-Untersuchung (DGPK 2014). 
Anamnese und klinische Untersuchung: Die ausführliche Erhebung der Krankengeschichte bezüglich Vorerkrankungen, herzerkrankungsspezifischen Symptomen sowie einer möglichen Herzinsuffizienzsymptomatik diente u.a. der Zuordnung der Patienten in die NYHA-Klassifikation. Diese ursprünglich von der New York Heart Association (NYHA) eingeführte Klassifikation dient der Einteilung von Herzerkrankungen, bzw. der Einteilung verschiedener Stadien der Herzinsuffizienz (I bis IV) entsprechend der Leistungsfähigkeit der Patienten mit erworbenen oder angeborenen Herzerkrankungen (Bredy et al. 2017). Symptome, die zur Beurteilung der Leistungsfähigkeit herangezogen werden sind Dyspnoe, Zyanose, Nykturie, Angina Pectoris, kalte Extremitäten, allgemeine Schwäche und Müdigkeit. Zusätzlich wurden die Patienten zu kardialen Symptomen wie vermehrtem Schwitzen bei Belastung oder in Ruhe, Palpitationen, Herzrasen oder Herzstolpern, Schwindel oder Synkopen befragt. Daraufhin erfolgte eine umfängliche körperliche Untersuchung mit Ermittlung von Gewicht und Körpergröße, der Errechnung des body mass index (BMI) sowie der Messung von Blutdruck, Herzfrequenz (HF) und transkutaner Sauerstoffsättigung $\left(\mathrm{SaO}_{2}\right)$. Zusätzlich erfolgte die Überprüfung der Reinheit der Herztöne bzw. das Feststellen von Herzgeräuschen mittels Stethoskop. Beurteilt wurden ebenso sonstige körperliche Auffälligkeiten, die für die Einschätzung des Ausmaßes der Krankheit relevant sind. Hierzu gehören beispielsweise Ödeme an den Extremitäten. Ein besonderes Augenmerk wurde hierbei auf die Form des Thorax und die Hände der Patienten gelegt um keine Hinweise für eine Sauerstoffunterversorgung zu übersehen. Um die Anamnese zu vervollständigen, befragten wir die Patienten zu ihrem Zigarettenkonsum, der Medikamenteneinnahme und ihrem wöchentlichen Sportpensum. Hierdurch erhielten wir einen detaillierten Eindruck des körperlichen Zustandes und des subjektiven Empfindens der Patienten hinsichtlich ihrer Herzerkrankung.

Blutentnahme und Laboruntersuchungen: Die laborchemischen Untersuchungen wurden im Zentrallabor der UMG durch Standarduntersuchungen bestimmt. Die ermittelten Werte gelten als Parameter der Routine-Verlaufsbeurteilung bei Patienten mit Herzerkrankungen. Zu den relevanten hämatologischen Parametern gehören: Blutbild mit Hämoglobinwert, Hämatokrit und Erythrozytenzahl. Elektrolyte wie Kalium, Calci- 
um, Chlorid und Magnesium sowie Parameter der klinischen Chemie, insbesondere der Leberwerte (Alanin-Aminotransferase (ALT), Aspartat-Aminotransferase (AST), Alkalische Phosphatase (AP), Gamma-Glutamyltransferase (y-GT), Glutamatdehydrogenase (GLDH), Laktatdehydrogenase (LDH), Cholinesterase (CHE)) und der Creatin-Kinase (CK) sowie der Creatin-Kinase Muscle-Brain (CK-MB) und der Troponin-I-Wert wurden ebenfalls bestimmt. Von besonderer Relevanz ist der Plasma-BNP Wert, da er als Herzinsuffizienzparameter anerkannt ist. Die Ergebnisse wurden je nach Alter und Geschlecht unterschiedlichen Referenzbereichen zugeordnet (Hösch 2015).

Apparative Untersuchungen: Das 12-Kanal-Ruhe-EKG wurde durch den Elektrokardiograph ELI ${ }^{\mathrm{TM}} 250$ und VERITAS ${ }^{\mathrm{TM}}$ Auswertungsalgorithmus (beide Mortara Instrument, Inc., Milwaukee, WI, USA) aufgenommen, die Schreibgeschwindigkeit betrug $25 \mathrm{~mm} / \mathrm{s}$. Die studienrelevanten Größen waren: HF, Rhythmus, Lagetyp, Erregungsausbreitungszeiten und Erregungsrückbildungszeiten (PQ-, QRS-, QTc-Dauer) sowie weitere im EKG sichtbare Veränderungen wie AV-Blöcke, P Pulmonale, Präexzitationen (Delta-Wellen), Extrasystolen, Schenkelblöcke und Hypertrophiezeichen (Hösch 2015).

Das 24-Stunden-Langzeit-EKG wurde mit dem Holter-Rekorder $\mathrm{H} 3^{\mathrm{TM}}$ aufgezeichnet und mit dem Analysesystem $\mathrm{H}-\mathrm{Scribe}^{\mathrm{TM}}$ ausgewertet (beide Mortara Instrument, Inc., Milwaukee, WI, USA). Analysiert wurden das Herzfrequenz-Spektrum, die Herzfrequenz-Variabilität sowie die Art, Häufigkeit und Dauer von Herzrhythmusstörungen und Pausen (Hösch 2015).

Die Spiroergometrie wurde mit dem Fahrrad-Spiroergometer ZAN ${ }^{\circledR} 600$ mit VIP'M ${ }^{\mathrm{TM}}$ (Variable Impedance Pneumotachograph) Fluss-Sensor ermittelt. Die Steigerung des Rampen-Protokolls betrug 20 Watt/Min. Die Daten wurden mit der ZAN ${ }^{\circledR}$-Tech Software (beides nSpire Health $\mathrm{GmbH}$, Oberthulba, Deutschland) ausgewertet. Die Lungenfunktion wurde anhand der Werte FEV1, IVC und FEV1/IVC beurteilt, die maximale Leistungsfähigkeit der Patienten wurde anhand der Messungen der maximalen Last, Herzfrequenz- und Blutdruckspektren, der maximalen Sauerstoffaufnahme $\left(\mathrm{VO}_{2}\right)$, des $\mathrm{O}_{2}$ Pulses und des exspiratorischen $\mathrm{CO}_{2}$-Quotienten $\left(\mathrm{VE} / \mathrm{VCO}_{2}\right)$ gemessen. Das Auftreten 
von Herzrhythmus- und Erregungsrückbildungsstörungen wurde ebenfalls registriert (Hösch 2015).

Diese spiroergometrischen Parameter wurden ausgewählt, da sie als zuverlässige Messgrößen der körperlichen Leistungsfähigkeit gelten. Die maximale $\mathrm{VO}_{2} / \mathrm{kg}$ ist die Menge an Sauerstoff, die in der Lunge aus der inspirierten Luft extrahiert wird. Sie gilt als klassische Messgröße zur Beurteilung der körperlichen Ausdauerleistungsfähigkeit. Das Atemäquivalent für Kohlendioxid $\left(\mathrm{VE} / \mathrm{VCO}_{2}\right)$ ist ein Marker für eine eingeschränkte Atemeffizienz. Es dient der Beurteilung der kardialen Auswurfleistung und der kardialen Leistungsreserve. Der Sauerstoffpuls $\left(\mathrm{VO}_{2} / \mathrm{HF}\right)$ gibt die Sauerstoffmenge an, die pro Herzschlag vom Körper extrahiert wird. Er ist eine anerkannte Kenngröße, um Aussagen über die Leistungsfähigkeit des Herzkreislaufsystems im Rahmen einer Leistungsdiagnose zu treffen (Van Gestel \& Teschler 2014).

Die echokardiographischen Untersuchungen wurden mit dem iE33 ${ }^{\text {TM }}$ Ultraschallsystem (Philips Healthcare, Leiden, Niederlande) durchgeführt und die Messungen elektronisch mit der Centricity Carddas Software (GE Healthcare, Fairfield, Connecticut, USA) im lokalen Bildarchivierungsarchiv der Klinik gespeichert. Die Messungen wurden im 2D-Echtzeitmodus und im M-Mode durchgeführt. Zusätzlich wurden Doppleruntersuchungen zur Messung von Flussrichtungen und Flussgeschwindigkeiten über den Klappen vorgenommen. Die Messungen erfolgten in den Standardherzachsen der Echokardiographie. Hierzu gehört die Darstellung des Herzens in der kurzen und langen Achse, der apikale Vier- und Fünfkammerblick, sowie die suprasternale und die subxiphoidale Achse. Flächen- und Volumenberechnungen erfolgten anhand der biplanen Methode nach Simpson. Epi- und endokardiale Grenzen wurden segmentiert, die ventrikuläre Masse, die Verkürzungsfraktion sowie die Ejektionsfraktion (EF) wurden berechnet und die Dyssynchronie des Septums und der Hinterwand des LV visuell beurteilt. Die Auswertung wurde mit der Image-Arena ${ }^{\mathrm{TM}}$ (TomTec, Unterschleißheim, Deutschland) und der Osirix ${ }^{\odot}$ Bildbearbeitungssoftware (Pixmeo Sarl, Bernex, Schweiz) durchgeführt (Hösch 2015).

Studienrelevante Größen waren die EF, die Versatzstrecke des TK-Ansatzes im Vergleich zum Mitralklappenansatz und die tricuspid annular plane systolic excursion (TAPSE). Die Messungen der Flächen von RA, aRV, fRV, LA und LV erfolgten im 4CV. 
Durch die ärztlichen Befunder erfolgte die optische Einteilung des TI-Grades. Außerdem wurden nebenbefundliche morphologische Normabweichungen und Herzfehlbildungen wie Septumdefekte vermerkt (Hösch 2015).

\subsection{Kardiale Magnetresonanztomographie}

Nach den Leitlinien für Patienten mit angeborenen Herzfehlern von den Deutschen Gesellschaften für Kardiologie und Pädiatrische Kardiologie sowie der Europäischen Gesellschaft für Kardiologie sind bei Patienten mit EA jährlich kardiologische Routineuntersuchungen empfohlen (Kilner et al. 2010). Im Rahmen dieser Routineuntersuchungen wurden bei dem Studienkollektiv zusätzlich MRT-Untersuchungen durchgeführt. Das hierzu verwendete Gerät ist ein 3-Tesla Skyra ${ }^{\circledR}$ MRT Scanner (Siemens Medical, Erlangen, Deutschland). Die Untersuchungszeit betrug für die Patienten je ca. 45 Minuten und erfolgte ohne Sedierung.

Die Aufnahmen folgten einem standartisierten EA-Protokoll, das der Vorgehensweise von 2013 entspricht um die Vergleichbarkeit zu gewährleisten. In dem Protokoll waren Standardsequenzen wie SSFP CINE-Aufnahmen im 4CV und Stapel in der ventrikulären und axialen kurzen Achse beinhaltet. Somit wurden alle Strukturen des Herzens inklusive der herznahmen Gefäße erfasst. Angewandt wurden hierbei folgende Einstellungen: Schichtdicke $=7 \mathrm{~mm}, \mathrm{TR}=49 \mathrm{~ms}, \mathrm{TE}=1,5 \mathrm{~ms}$, Flipwinkel $=50^{\circ}$, räumliche Auflösung max. 1,3 x 1,3 x 7,0 mm (Voxelgröße), ein paralleler Bildgebungsbeschleunigungsfaktor von 2 sowie ein retrospektives Gating und 25 Phasen pro Herzzyklus abhängig von der Herzfrequenz. Aus Stapeln, die mittels Multislice-Multiphasen-Technik entstanden, wurden die Herzkammervolumina, Funktionsparameter und die Herzmuskelmasse ermittelt. Die Aufnahmen wurden in Atemmittellage aufgenommen und zeigen die axiale Ebene (Hösch 2015).

Die Segmentierung der einzelnen Schichten im axial geschichteten Stapel wurde mit QMass $^{\odot}$ und Visia ${ }^{\odot}$ Software (Medis, Leiden, Niederlande) durchgeführt, ebenso wie die Berechnungen der Flächen und der TK-Versatz im 4CV. Die epi- und endokardialen Grenzlinien wurden manuell in der Enddiastole und Endsystole für das RA, den aRV, den fRV, das LA und den LV von einem Befunder eingezeichnet.

In Abbildung 4 wird die Segmentierung der einzelnen Herzkammern dargestellt. 


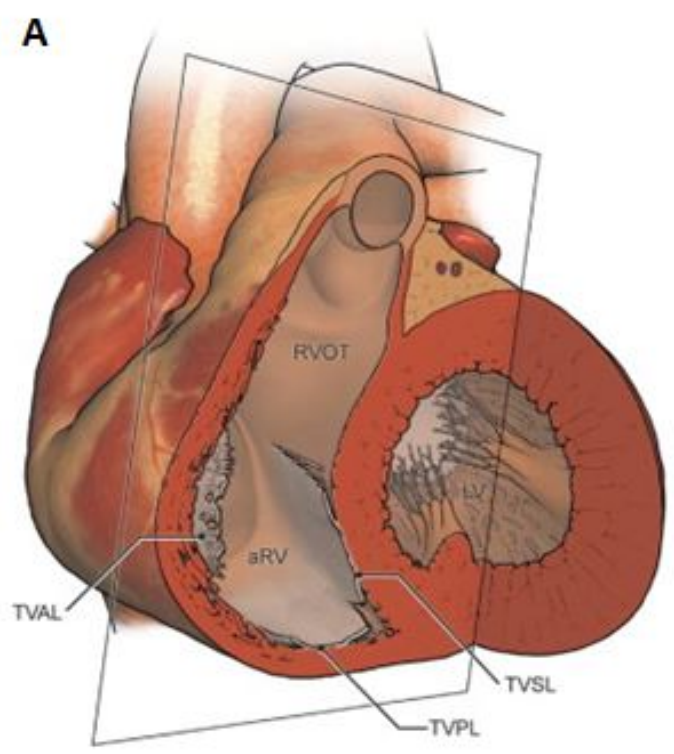

B
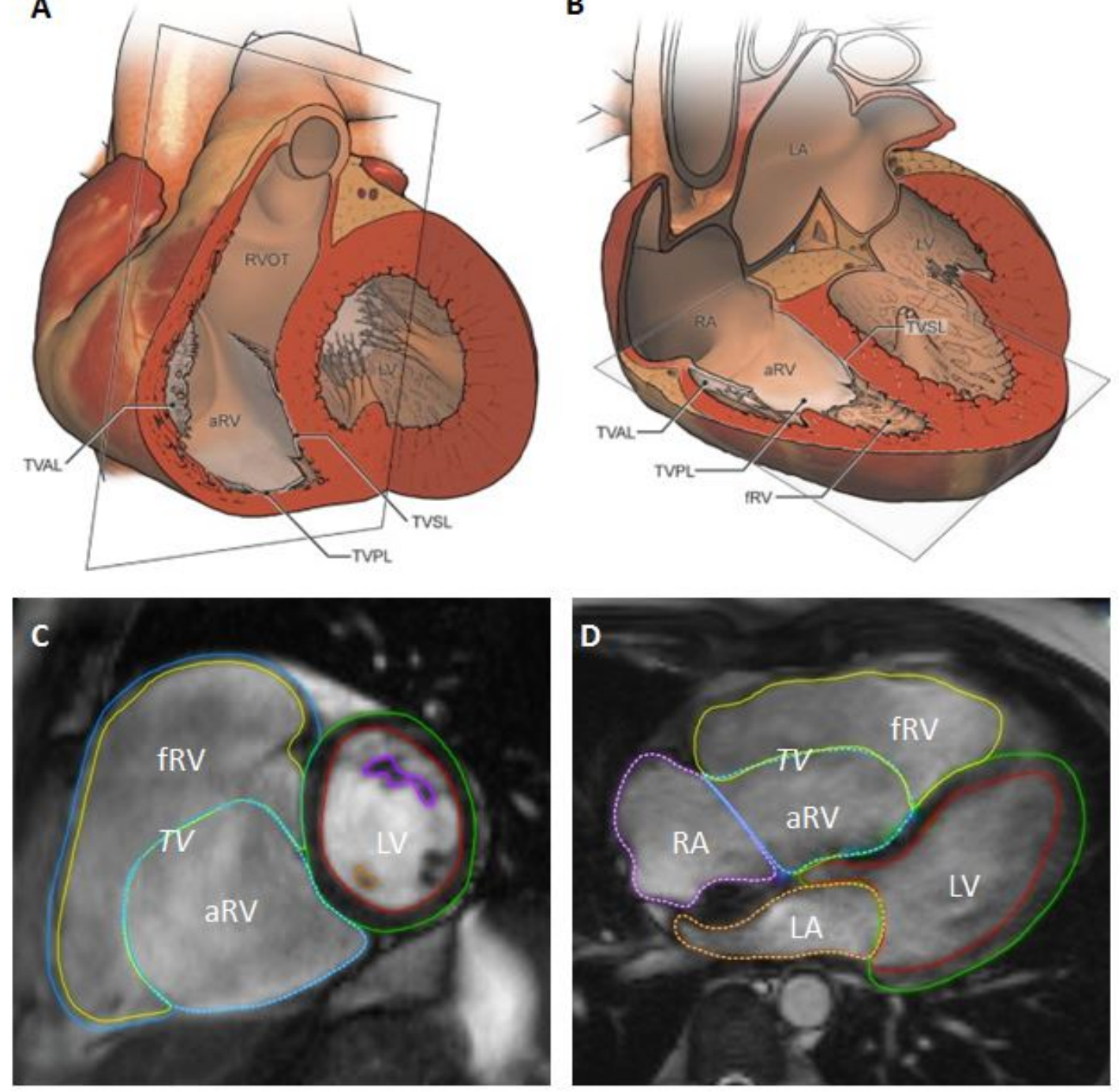

Abbildung 4: Dreidimensionale Reproduktion der Ebstein Anomalie (Yalonetsky et al. 2011) und Segmentierungsregeln der cMRT-Bilder (Hösch et al. 2014) in der kurzen Achse und in axialer Ausrichtung (mit freundlicher Genehmigung der Autoren und des Elsevier-Verlags).

Darstellung in der kurzen Achse: Bild A, C; Darstellung in axialer Ausrichtung: Bild B, D.

$\mathrm{aRV}=$ atrialisierter rechter Ventrikel; $\mathrm{fRV}=$ funktioneller rechter Ventrikel, $\mathrm{LA}=$ linker Vorhof; $\mathrm{LV}=$ linker Ventrikel; RA = rechter Vorhof; RVOT = rechts-ventrikulärer Ausflusstrakt; TV = Trikuspidalklappe; TVAL = anteriores Segel der Trikuspidalklappe; TVSL = septales Segel der Trikuspidalklappe; TVPL = posteriores Segel der Trikuspidalklappe.

Die Grenzlinien wurden randomisiert und verblindet von einem weiteren erfahrenen ärztlichen Befunder kontrolliert. Als relevante Parameter für die Studie wurden das enddiastolische Volumen (EDV), das endsystolische Volumen (ESV), das Schlagvolumen 
(SV), die EF, das HZV, die Muskelmasse sowie der R/L-VI bestimmt. Die Flussmessung erfolgte mittels QFlow ${ }^{\complement}$ Software (Medis, Leiden, Niederlande) (Hösch 2015).

Die cMRT gilt heutzutage laut zahlreichen Studien als Referenzstandard für die Analyse der Ausmaße und Funktion des LV und RV (Dewey et al. 2006; Zhou et al. 2015). Für die genaue Ermittlung der Volumina der rechten Herzkammern gibt es bei EAPatienten derzeit allerdings keine definierten Standards. Laut den Vorgaben von 2013 wurde in dieser Studie der RA vom aRV durch das Einzeichnen einer gerade Fläche auf der Ursprungsebene des Segelklappenannulus unterschieden. Die TK wurde im Detail nachverfolgt und ihr Verlauf in jeder Schicht genau eingezeichnet um den aRV vom fRV abzugrenzen. Die Segmentierung des LA und des LV erfolgte in der kurzen Achse gemäß den Standards der American Heart Association (Cerqueira et al. 2002).

Eine vereinfachte Berechnung des Totalen Rechts/Links-Volumen-Index ist allerdings ebenfalls möglich.

Um den R/L-VI einfach und ohne größeren Zeitaufwand ermitteln zu können, ist es nicht nötig, die jeweiligen Herzkammern getrennt voneinander und den Verlauf der Segelklappen im Detail einzuzeichnen. Stattdessen kann der Index auch durch das Umfahren der Gesamtkontur der jeweiligen Herzhälfte ermittelt werden. Dadurch entfällt die aufwendige Segmentierung jeder einzelnen Herzkammer, welche bei der EA vor allem auf der rechten Herzseite problematisch ist. Für die Berechnung des Index genügt es, das Gesamtvolumen der rechts- und linksseitigen Herzkammern zu bestimmen ohne die dysplastische TK als Grenze zwischen dem aRV und dem fRV einzuzeichnen. Dies vereinfacht die Auswertung und Ermittlung des Index deutlich. Diese vereinfachte Segmentierung wurde für alle Patienten ebenfalls durchgeführt.

In Abbildung 5 wird auf den zwei linken Bildern die Segmentierung der einzelnen Kompartimente des Herzens ersichtlich. Auf den beiden rechtsseitigen Bildern wird die vereinfachte Kontureneinzeichnung zur Ermittlung des Totalen Rechts/Links-VolumenIndex dargestellt. 

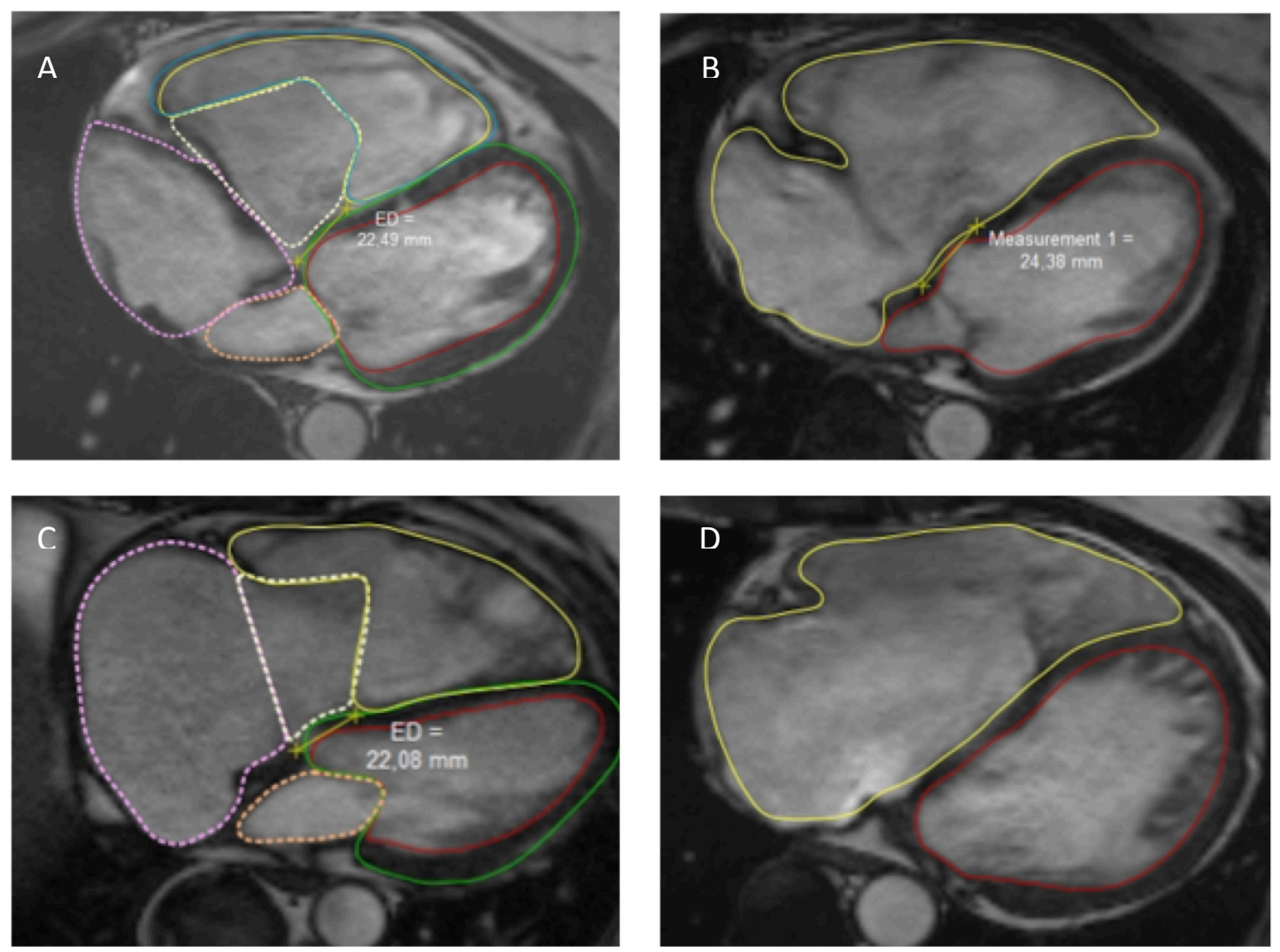

Abbildung 5: Segmentierung der einzelnen Kompartimente des Herzens und vereinfachte Kontureneinzeichnung zur Ermittlung des Totalen Rechts/Links-Volumen-Index.

Links: Segmentierung der einzelnen Kompartimente des Herzens (Bild A, C); Rechts: vereinfachte Kontureneinzeichnung zur Ermittlung des Rechts/Links-Volumen-Index (Bild B, D).

A, C) Formel zur Errechnung des Rechts/Links-Volumen-Index aus der Segmentierung der einzelnen Kompartimente des Herzens:

$$
\begin{aligned}
R / L-V I= & (R A+a R V+f R V) /(L A+L V): \\
& \frac{(54+73+109)}{(16+57)=3,2}
\end{aligned}
$$

B, D) Formel zur Errechnung des Rechts/Links-Volumen-Index mittels der vereinfachten Kontureneinzeichnung:

$$
\mathrm{R} / \mathrm{L}-\mathrm{VI}=\mathrm{RHV} / \mathrm{LHV} \text { : }
$$

$$
73=3,2
$$




\subsection{Statistische Analysen}

Mittels den Computerprogrammen Statistica ${ }^{\circledR}$ (Stat Weich, North Melbourne, Australien), SPSS ${ }^{\circledR} 23$ (IBM Corporations, Armonk, New York, USA), GraphPad Prism ${ }^{\circledR} 7.0$ (GraphPad Software, La Jolla, California, USA) und Microsoft Excel ${ }^{\circledR}$ (Microsoft Corporation, Redmond, Washington, USA) erfolgte die statistische Auswertung der Daten. Hierbei arbeiteten wir mit dem Institut für Medizinische Statistik der UMG zusammen. Sie beinhaltete deskriptive Statistiken, Korrelationskoeffizienten nach der PearsonMethode für kontinuierliche Variablen und die Spearman-Rangfolgen-Korrelation für ordinale Variablen (NYHA, $\mathrm{TI}^{\circ}$ visuell) samt den entsprechenden 95\%Konfidenzintervallen (nach Fisher-Transformation) und $p$-Werten des Tests $r=0$ (Hösch 2015).

Um die Reliabilität und Objektivität der Berechnung des Totalen R/L-VI zu überprüfen, wurden Inter- und Intraobservertests durchgeführt. Die Durchführung der Inter- und Intraobservertests erfolgte sechs Wochen nach den ersten Auswertungen anhand von sechs Datensets (30\%). Diese sechs zufällig ausgewählten MRT-Bildersets wurden von dem gleichen Befunder (Intraobserver) und einem weiteren Befunder (Interobserver) unabhängig voneinander in einer verblindeten und randomisierten Form ausgewertet. Hierzu wurden die endokardialen und epikardialen Grenzen von beiden Befundern manuell eingezeichnet.

Um die Ergebnisse der Inter- und Intraobserver-Variabilitäten beurteilen zu können, wurde der Variationskoeffizient (engl. Coefficient of Variation, CoV, Angabe in \%) und der Intraklassen-Korrelations-Koeffizient (engl. Intraclass Correlation Coefficient, ICC) errechnet.

Der Variationskoeffizient ist ein relatives Streuungsmaß, das nicht von der Maßeinheit der statistischen Variablen abhängt (Kohn 2005). Er ist somit eine Normierung der Varianz und für Interrater-Untersuchungen gut geeignet (Kohn 2005).

Als weiteres Verfahren zur Ermittlung des Übereinstimmungsgrades zwischen mehreren Befundern bezogen auf verschiedene Untersuchungsobjekte wurde die IntraKlassen-Korrelation angewandt. Der ICC wird zum Vergleich von mehrere Beobachtungszeitpunkten berechnet (Shrout \& Fleiss 1979; Wirtz \& Caspar 2002). 
Zudem wurden Bland-Altman-Diagramme erstellt um den Vergleich der zwei Messungen der Befunder graphisch darzustellen. Anhand dieser ist es möglich die Schwankungsbreite der Abweichung einzusehen und systematische Messfehler zu erkennen (Bland \& Altman 1986).

Es wurde außerdem für verschiedene Schwergrade der $E A(R / L-V I>2,0$ und $<2,0)$ eine Kaplan-Meier-Kurve hinsichtlich kardiovaskulärer Ereignisse erstellt um den Nutzen des Index zur Risikoberechnung für das Eintreten kardialer Ereignisse zu testen.

Das Kaplan-Meier-Verfahren wird in dieser Studie angewendet um die Wahrscheinlichkeit zu schätzen, mit der bei einem Patienten mit einem Risikofaktor ein kardiales Ereignis innerhalb eines bestimmten Zeitraumes eintritt. Als Risikofaktor gilt ein R/L-VI von > 2,0. Als Maß für den Risikovergleich zweier Gruppen wurde das Hazard Ratio (HR) berechnet. Als deskriptive Größe dient es dem Vergleich von Überlebenszeiten verschiedener Patientengruppen und wird wie ein relatives Risiko interpretiert (Kaplan \& Meier 1985).

Zusätzlich wurde eine Regressionsanalyse durchgeführt um die Aussagekraft des Index als prognostischen Vorhersageparameter zu evaluieren. Dieses statistische Verfahren untersucht nicht nur die Beziehung zwischen mehreren unabhängigen Variablen und einer abhängigen Variablen, sondern zielt ebenso darauf ab, den Zusammenhang quantitativ zu beschreiben und Werte der abhängigen Variablen zu prognostizieren (Schneider et al. 2010). Die Regressionsanalyse diente dazu, den Zusammenhang einer möglichen Veränderung des R/L-VI und einer Veränderung der übrigen Parameter zu untersuchen. Bei der Regressionsanalyse ist der Regressionskoeffizient b die Steigung der Regressionsgeraden und der Regressionskoeffizient für die Stichprobe. An ihm lässt sich der Beitragsanteil der Änderung des R/L-VI (Einflussvariable) für die Erklärung der Änderung der klinischen Parameter (Zielgröße) ablesen. Wenn die Einflussgröße konstant ist, beschreibt der Regressionskoeffizient die Veränderung der Zielvariablen pro Maßeinheit der Einflussvariablen (Schneider et al. 2010). Die multivariable Regressionsanalyse ermöglicht es nicht nur mehrere Einflussgrößen gleichzeitig zu betrachten, sondern auch die Regressionskoeffizienten der Einflussgrößen bezüglich möglicher Störfaktoren zu adjustieren (Schneider et al. 2010). Hierzu wird der standartisierte Regressionskoeffizient $b^{*}$ angegeben. Das so genannte Bestimmtheitsmaß $R^{2}$ drückt aus, 
wie gut die Regressionsgerade den Zusammenhang zwischen der unabhängigen und der abhängigen Variablen wiedergibt. Das $\mathrm{R}^{2}$ gibt somit den Anteil der Varianz der abhängigen Variablen an, der durch die unabhängigen Variablen erklärt werden kann. Das Bestimmtheitsmaß ist ein Gütemaß der linearen Regression, es liegt zwischen 0 bzw. 0\% (unbrauchbares Modell) und 1 bzw. 100\% (perfekte Modellanpassung) (Schneider et al. 2010).

Weiterhin wurde der Einfluss von den Parametern Alter und Geschlecht auf die Veränderung des R/L-VI (abhängige Variable) untersucht. 


\section{Ergebnisse}

\subsection{Ergebnisse der Untersuchungen}

Von 21 Patienten, welche die Einschlusskriterien erfüllten, wurden alle Daten erfasst und analysiert. 29\% der Teilnehmer waren weiblich und $71 \%$ waren männlich. Das Durchschnittsalter der Patienten betrug zum Untersuchungszeitpunkt $28 \pm 16$ Jahre, ein Drittel der Patienten war unter 18 Jahre alt. Patientenmerkmale und die klinisch relvanten Untersuchungsparameter zur Beurteilung der EA sind in Tabelle 1 zusammengefasst.

Tabelle 1: Patientenmerkmale und klinische Parameter 2016.

\begin{tabular}{|c|c|c|c|}
\hline \multicolumn{2}{|c|}{ Studienkohorte $(n=21)$} & \multirow{2}{*}{$\begin{array}{l}\text { Mittelwert } \pm \text { SD } \\
28 \pm 16\end{array}$} & \multirow{2}{*}{$\begin{array}{l}\text { Median (Spanne) } \\
23(13-63)\end{array}$} \\
\hline Klinische Daten & Alter (Jahre) & & \\
\hline & $\mathrm{BMI}\left(\mathrm{kg} / \mathrm{m}^{2}\right)$ & $26 \pm 8$ & $23(17-48)$ \\
\hline & $\mathrm{SaO}_{2}(\%)$ & $99 \pm 2$ & $99(95-100)$ \\
\hline & NYHA (n (\%)) & I: 12 (57), II: 7 (33), & III: 2(10), IV: $0(0)$ \\
\hline \multirow[t]{2}{*}{ Labor } & $\mathrm{BNP}(\mathrm{ng} / \mathrm{l})$ & $46 \pm 78$ & $14,3(10-354)$ \\
\hline & BNP > $100(n g / l)(n(\%))$ & $3(14 \%)$ & \\
\hline \multirow[t]{2}{*}{ EKG } & QRS-Dauer (ms) & $113 \pm 24$ & $110(80-180)$ \\
\hline & QTc-Dauer (ms) & $415 \pm 27$ & $405(367-475)$ \\
\hline \multirow[t]{3}{*}{ Spiroergometrie } & Max. $\mathrm{VO}_{2}$ (\% vom Sollwert) & $73 \pm 19$ & $71(35-112)$ \\
\hline & Max. VE/VCO 2 (\% vom Sollwert) & $112 \pm 15$ & 109 (87-147) \\
\hline & Max. $\mathrm{O}_{2}$-Puls (\% vom Sollwert) & $88 \pm 27$ & $83(51-175)$ \\
\hline \multirow[t]{4}{*}{ Echokardiographie } & TI visuell $\geq$ moderat $(\mathrm{n}(\%))$ & $15(71)$ & \\
\hline & TK-Versatz $\left(\mathrm{mm} / \mathrm{m}^{2}\right)$ & $35 \pm 12$ & $36(17-55)$ \\
\hline & TAPSE (cm) & $2,7 \pm 1$ & $2,7(0,6-5,2)$ \\
\hline & LV-EF (biplan, Simpson) (\%) & $57 \pm 9$ & $57(43-71)$ \\
\hline
\end{tabular}

$\mathrm{BMI}=$ body mass index $; \mathrm{BNP}=$ brain natriuretic peptide $; \mathrm{LV}-\mathrm{EF}=$ linksventrikuläre Ejektionsfraktion; Max. = Maximale; NYHA = New York Heart Association; $\mathrm{SaO}_{2}=$ Sauerstoffsättigung; $\mathrm{SD}=$ Standardabweichung; TAPSE = tricuspid annular plane systolic excursion; $\mathrm{TI}=$ Trikuspidalklappeninsuffizienz; TK = Trikuspidalklappe; $\mathrm{VE} / \mathrm{VCO}_{2}=$ exspiratorischer $\mathrm{CO}_{2}$-Quotient; $\mathrm{VO}_{2}=$ maximale Sauerstoffaufnahme. 
Der durchschnittliche Beobachtungszeitraum bis zur ersten Folgeuntersuchung betrug 36,3 $\pm 5,1$ Monate bzw. 3,0 $\pm 0,4$ Jahre (Mittelwert \pm Standardabweichung).

Der durchschnittliche BMI-Wert betrug $26 \mathrm{~kg} / \mathrm{m}^{2}$ und lag somit im übergewichtigen Bereich. Das Gewicht von zehn Patienten war als normal einzustufen, zwei der Studienteilnehmer waren untergewichtig, fünf übergewichtig, zwei fettleibig und zwei wurden als krankhaft übergewichtig eingestuft.

Anhand der Untersuchungsergebnisse ließen sich bei vielen Patienten deutliche Hinweise für Rechts- und Linksherzinsuffizienz nachweisen.

In den Laboruntersuchungen wurde hierzu der Plasma-BNP-Wert bestimmt, welcher als Herzinsuffizienzmarker gilt. Pathologische Werte über der Herzinsuffizienzschwelle von über $100 \mathrm{ng} / \mathrm{l}$ wurden bei drei der 21 Teilnehmer (14\%) gemessen. Der niedrigste BNP-Wert betrug < $10 \mathrm{ng} / \mathrm{l}$, der höchste $354 \mathrm{ng} / \mathrm{l}$.

Bei allen Studienteilnehmern zeigte das EKG einen Sinusrhythmus. Die QRS-Dauer war bei 13 Patienten (59\%) auf > 110 ms und die QTc-Zeit bei 14\% auf > 440 ms verlängert. Ein kompletter Rechtsschenkelblock wurde bei sieben Studienteilnehmern (32\%) festgestellt.

In der 24-Stunden-LZ-EKG-Analyse zeigten sich bei zehn Patienten ventrikuläre (monosowie polymorphe), bei fünf Patienten supraventrikuläre Extrasystolen.

Bei einer Patientin des Kollektivs wurde im 24-Stunden-Untersuchungszeitraum eine supraventrikuläre Tachykardie bei Präexzitationen aufgezeichnet. Bei zwei weiteren Patienten traten anamnestisch Palpitationen und Tachykardien auf, die eine EPU zur Folge hatten. In der EPU wurde die erste Tachykardie als supraventrikuläre Tachykardie mit dualen AV-Knoten-Leitungseigenschaften identifiziert. Eine zweite Patientin entwickelte supraventrikuläre Tachykardien, später Vorhofflattern und zuletzt permanentes Vorhofflimmern.

Die Spirometrie ergab eine unauffällige bzw. gute Lungenfunktion bei dem gesamten Studienkollektiv, der FEV1-Wert lag allerdings bei vier Patienten (19\%) unter 80\%. Die Spiroergometrie ergab weiterhin auffällig erniedrigte Werte für die maximale $\mathrm{VO}_{2} / \mathrm{kg}$ bei 15 Patienten (71\%). Bei diesen lagen die Werte unter $85 \%$ vom Sollwert. Das Atemäquivalent für Kohlendioxid $\mathrm{VE} / \mathrm{VCO}_{2}$ war bei sieben Personen (33\%) auf $>30 \mathrm{~L} / \mathrm{L}$ er- 
höht. Der maximale $\mathrm{O}_{2}$ Puls war bei allen Patienten unauffällig (Normwert bei maximaler Belastung: 8 +/- 14 ml Sauerstoff pro Herzschlag).

Bei der echokardiographischen Untersuchung fiel bei 15 Studienteilnehmern (68\%) eine mäßig bis schwere TI auf. Der TK-Versatz betrug im Durchschnitt 3,5 cm. Zur Beurteilung der longitudinalen systolischen RV-Funktion wurde die TAPSE echokardiographisch gemessen. Diese hatte eine Spannweite von $0,6 \mathrm{~cm}-5,2 \mathrm{~cm}$ und war bei fünf Patienten mit $<2 \mathrm{~cm}$ erniedrigt, bei einem Studienteilnehmer allerdings mit $>4 \mathrm{~cm}$ deutlich über das normale Maß hinaus erhöht. Bei neun Teilnehmern (43\%) war die biplane LV-Funktion (modifiziert nach Simpson) mit $<60 \%$ erniedrigt.

Fünf der Patienten hatten einen ASD, drei weitere ein PFO. Fünf dieser Defekte waren mittels Occluder verschlossen worden, zwei durch einen Direktverschluss. Ein Patient hatte zum Untersuchungszeitpunkt weiterhin ein unverschlossenes PFO.

Zum exakten Vergleich der Patientenmerkmale und der Ergebnisse der klinischen Untersuchungsparameter der Jahre 2013 und 2016 dient die folgende Tabelle (Tabelle 2). Alle Daten beziehen sich auf das gleiche Patientenkollektiv bestehend aus 21 Patienten, welche sowohl im Jahr 2013 als auch im Jahr 2016 zu den Untersuchungen erschienen. 
Tabelle 2: Patientenmerkmale und klinische Parameter 2013 und 2016 im Vergleich.

\begin{tabular}{|c|c|c|c|c|}
\hline \multirow{2}{*}{\multicolumn{2}{|c|}{ Studienkohorte $(n=21)$}} & \multirow{3}{*}{$\begin{array}{l}\text { Mittelwert } \pm \text { SD } \\
2013 \\
25 \pm 16\end{array}$} & \multirow{2}{*}{$\begin{array}{l}\text { Mittelwert } \pm \text { SD } \\
2016\end{array}$} & \multirow{3}{*}{$\begin{array}{r}p- \\
\text { Wert } \\
<0,001\end{array}$} \\
\hline & & & & \\
\hline \multirow[t]{9}{*}{ Klinische Daten } & Alter (Jahre) & & $28 \pm 16$ & \\
\hline & $\mathrm{BMI}\left(\mathrm{kg} / \mathrm{m}^{2}\right)$ & $25 \pm 7$ & $26 \pm 8$ & 0,015 \\
\hline & Größe & $171 \pm 13$ & $175 \pm 8$ & 0,027 \\
\hline & Gewicht & $73 \pm 25$ & $80 \pm 26$ & 0,003 \\
\hline & BSA & $1,8 \pm 0,4$ & $2 \pm 0,3$ & 0,004 \\
\hline & NYHA (Anzahl n (\%)) & I: $16(76)$ & I: $13(62)$ & \\
\hline & & II: 3 (14) & II: 6 (28) & \\
\hline & & III: 2 (10) & III: 2 (10) & \\
\hline & & IV: $0(0)$ & IV: $0(0)$ & \\
\hline \multirow[t]{2}{*}{ Labor } & $\mathrm{BNP}(\mathrm{ng} / \mathrm{l})$ & $42 \pm 47$ & $46 \pm 78$ & 0,663 \\
\hline & BNP $>100(n g / l) n(\%)$ & $4(19)$ & $3(14)$ & \\
\hline \multirow[t]{2}{*}{ EKG } & QRS-Dauer (ms) & $118 \pm 29$ & $113 \pm 24$ & 0,401 \\
\hline & QTc-Dauer (ms) & $414 \pm 28$ & $415 \pm 27$ & 0,887 \\
\hline \multirow[t]{3}{*}{ Spiroergometrie } & Max. $\mathrm{VO}_{2}$ (\% vom Sollwert) & $69 \pm 16$ & $73 \pm 19$ & 0,100 \\
\hline & VE $/ \mathrm{VCO}_{2}$ (\% vom Sollwert) & $111 \pm 15$ & $112 \pm 15$ & 0,692 \\
\hline & Max. $\mathrm{O}_{2}$-Puls (\%) & $82 \pm 16$ & $88 \pm 27$ & 0,497 \\
\hline \multirow[t]{5}{*}{ Echokardiographi } & & & & \\
\hline & irvisuen = moderda (ming) & $10(1 /)$ & $10(11)$ & \\
\hline & TK-Versatz $\left(\mathrm{mm} / \mathrm{m}^{2}\right)$ & $37 \pm 92$ & $35 \pm 12$ & 0,438 \\
\hline & TAPSE $(\mathrm{cm})$ & $2,6 \pm 1$ & $2,7 \pm 1$ & 0,447 \\
\hline & LV-EF (biplan, Simpson) (\%) & $59 \pm 6$ & $57 \pm 9$ & 0,398 \\
\hline \multirow[t]{3}{*}{ Ereignisse } & NYHA-Klassen-Änderung ( $n$ ) & 3 & & \\
\hline & Tachykardien (n) & 3 & & \\
\hline & Todesfälle (n) & 1 & & \\
\hline
\end{tabular}

$\mathrm{BMI}=$ body mass index $\mathrm{BNP}=$ brain natriuretic peptide; $\mathrm{BSA}=$ body surface area $; \mathrm{LV}-\mathrm{EF}=$ linksventrikuläre Ejektionsfraktion; Max. $=$ Maximale; NYHA = New York Heart Association; $\mathrm{SaO}_{2}=$ Sauerstoffsättigung; SD = Standardabweichung; TAPSE = tricuspid annular plane systolic excursion; $\mathrm{TI}=$ Trikuspidalklappeninsuffizienz; $\mathrm{TK}=$ Trikuspidalklappe; $\mathrm{VE} / \mathrm{VCO}_{2}=$ exspiratorischer $\mathrm{CO}_{2}$-Quotient; $\mathrm{VO}_{2}=$ maximale Sauerstoffaufnahme.

Fett gedruckt: $p<0,05$ (signifikante Ergebnisse). 
Drei Jahre nach Studienbeginn wurde die erste Wiederholung der klinischen Untersuchungen sowie der MRT-Untersuchungen angesetzt, um möglichst frühzeitig Veränderungen des klinischen Zustands der Patienten feststellen zu können. Vor allem die Größe und das Gewicht einiger minderjähriger Patienten hatten sich in dieser Zeit deutlich verändert. Somit ergaben sich statistisch signifikante Unterschiede in den Patientenmerkmalen Alter, Gewicht, Größe, BMI und body surface area (BSA). Der restliche Vergleich der Patientenmerkmale und klinischen Untersuchungen ergab von 2013 bis 2016 allerdings kaum signifikante Unterschiede.

In der körperlichen Untersuchung fielen bei sieben Patienten neue Symptome wie Dyspnoe bei Belastung, Schwindel, Schweißausbrüche oder Ödeme auf. Drei Patienten gaben 2016 erstmalig an, Palpitationen bemerkt zu haben.

Drei Patienten, die 2013 noch der NYHA-Klasse I zugeordnet worden waren, wurden drei Jahre später in die NYHA-Klasse II eingestuft. Die körperliche Belastbarkeit der Patienten hatte sich innerhalb dieser Zeit subjektiv verschlechtert. 2013 hatten die drei Erkrankten angegeben keine Einschränkungen in ihrer Belastbarkeit festzustellen, drei Jahre später bemängelten sie leichte Einschränkungen und das Auftreten von Herzinsuffizienzsymptomen bei starker körperlicher Belastung.

Die Sauerstoffsättigung im Blut zeigte keine merklichen Änderungen zu den vor drei Jahren gemessenen Werten. Der durchschnittliche Plasma-BNP-Wert veränderte sich im Kollektiv kaum, jedoch war der Wert bei neun Patienten leicht gesunken und bei sechs Studienteilnehmern gestiegen, bei drei von ihnen deutlich. Bei insgesamt 3 von 21 Studienteilnehmern ließ sich erneut ein pathologisch erhöhter Plasma-BNP-Wert nachweisen. Der höchste Plasma-BNP-Wert von 2016 betrug 354,3 ng/l, drei Jahre zuvor hatte der gleiche Studienteilnehmer einen Plasma-BNP-Wert von $173 \mathrm{ng} / \mathrm{l}$. Der Patient mit dem höchsten BNP-Wert von 2013 (546,5 ng/l) war im Zeitraum von 2013 bis 2016 verstorben.

Im 12-Kanal-Ruhe-EKG ergaben sich ebenfalls keine Unterschiede zu den Voruntersuchungen. Weder die QRS-Dauer noch die QTc-Dauer hatten sich im Kollektiv signifikant verändert. 
In den LZ-EKG-Untersuchungen wurden bei zwei Studienteilnehmern neu aufgetretene ventrikuläre Extrasystolen aufgezeichnet, bei fünf Patienten wurde eine deutlich erhöhte Zahl an ventrikuläre Extrasystolen notiert.

Ebenso zeigten sich sowohl in der Spiroergometrie als auch in der Echokardiographie kaum Veränderungen zu den Voruntersuchungen. Die FEV1/IVC Werte verbesserten sich im Kollektiv tendenziell leicht aber nicht signifikant, während die IVC bei insgesamt drei Patienten 2016 unter 70\% lag.

Weder der maximale $\mathrm{VO}_{2}(\%)$, noch die $\mathrm{VE} / \mathrm{VCO}_{2}(\%)$ noch der maximale $\mathrm{O}_{2}$-Puls (\%) zeigten signifikante Unterschiede zu den vor drei Jahren gemessenen Werten.

In der Echokardiographie ergaben sich vergleichbare Werte für die TAPSE sowie für die LV-EF nach Simpson (n.S.) (biplan). Der deutliche TK-Versatz, der typisch für Patienten mit EA ist, wurde wiederholt gemessen und in seinem Ausmaß bei allen Patienten bestätigt. Lediglich die TI wurde visuell von den ärztlichen Befundern bei einem Patienten als höhergradig eingestuft.

Trotz den insgesamt kaum signifikanten Veränderungen in den klinisch messbaren Parametern des Kollektivs zeigt eine genauere Betrachtung einzelner Studienteilnehmer kleine messbare Unterschiede bereits drei Jahre nach den ersten Untersuchungen. Zusätzlich sind innerhalb dieser Zeit einige für diese Studie relevante kardiovaskuläre Ereignisse aufgetreten. Drei Teilnehmer wurden in eine höhere NYHA-Klasse eingestuft $(I \rightarrow$ II) und drei Patienten hatten innerhalb des Beobachtungszeitraumes Tachykardien entwickelt. Ein Patient war kurz nach den ersten Untersuchungen von 2013 verstorben.

\subsection{Ergebnisse der MRT-Untersuchungen}

Die folgende Tabelle (Tabelle 3) zeigt die Ergebnisse der mittels MRT errechneten Funktionsparameter und der Herzkammervolumina des gepaarten Kollektivs im Jahr 2013 und 2016. 
Tabelle 3: Die berechnetes cMRT-Funktionsparameter und Herzkammervolumina von 2013 und $2016 \mathrm{im}$ Vergleich.

\begin{tabular}{|c|c|c|c|c|}
\hline \multicolumn{2}{|c|}{ Studienkohorte $(n=21)$} & \multirow{2}{*}{$\begin{array}{l}2013 \\
\text { (Mittelwert } \pm \text { SD) } \\
22 \pm 11\end{array}$} & \multirow{2}{*}{$\begin{array}{l}2016 \\
\text { (Mittelwert } \pm \text { SD) } \\
20 \pm 8\end{array}$} & \multirow{2}{*}{$\begin{array}{r}\text { p-Wert } \\
0,080\end{array}$} \\
\hline Funktions- & TK-Versatz $\left(\mathrm{mm} / \mathrm{m}^{2}\right)$ & & & \\
\hline \multirow[t]{4}{*}{ parameter } & fRV-EF > 48\% (\%) & $46 \pm 8$ & $39 \pm 12$ & 0,006 \\
\hline & LV-EF > 48\% (\%) & $56 \pm 6$ & $54 \pm 9$ & 0,116 \\
\hline & Qs $\left(I / \min \cdot \mathrm{m}^{2}\right)$ [Fluss] & $2,9 \pm 0,6$ & $2,8 \pm 0,7$ & 0,469 \\
\hline & $\mathrm{Qp}\left(\mathrm{I} / \mathrm{min} \cdot \mathrm{m}^{2}\right)$ [Fluss] & $3,6 \pm 0,9$ & $3,2 \pm 1,3$ & 0,208 \\
\hline \multicolumn{5}{|l|}{ Volumina } \\
\hline \multirow[t]{6}{*}{ Rechtes Herz } & RA EDVi $\left(\mathrm{ml} / \mathrm{m}^{2}\right)$ & $103 \pm 36$ & $108 \pm 38$ & 0,355 \\
\hline & RA ESVi $\left(\mathrm{ml} / \mathrm{m}^{2}\right)$ & $54 \pm 27$ & $64 \pm 33$ & 0,002 \\
\hline & aRV EDVi $\left(\mathrm{ml} / \mathrm{m}^{2}\right)$ & $52 \pm 35$ & $52 \pm 34$ & 0,639 \\
\hline & aRV ESVi $\left(\mathrm{ml} / \mathrm{m}^{2}\right)$ & $30 \pm 28$ & $28 \pm 24$ & 0,152 \\
\hline & fRV EDVi $\left(\mathrm{ml} / \mathrm{m}^{2}\right)$ & $112 \pm 31$ & $125 \pm 37$ & 0,018 \\
\hline & fRV ESVi $\left(\mathrm{ml} / \mathrm{m}^{2}\right)$ & $60 \pm 20$ & $75 \pm 24$ & 0,007 \\
\hline \multirow[t]{4}{*}{ Linkes Herz } & LA EDVi $\left(\mathrm{ml} / \mathrm{m}^{2}\right)$ & $32 \pm 9$ & $34 \pm 8$ & 0,093 \\
\hline & LA ESVi $\left(\mathrm{ml} / \mathrm{m}^{2}\right)$ & $18 \pm 5$ & $21 \pm 7$ & 0,009 \\
\hline & LV EDVi $\left(\mathrm{ml} / \mathrm{m}^{2}\right)$ & $72 \pm 10$ & $76 \pm 14$ & 0,042 \\
\hline & LV ESVi $\left(\mathrm{ml} / \mathrm{m}^{2}\right)$ & $31 \pm 7$ & $35 \pm 10$ & 0,026 \\
\hline
\end{tabular}

$\mathrm{aRV}=$ atrialisierter rechter Ventrikel; EDVi = enddiastolisches Volumen geindext; $\mathrm{EF}=$ Ejektionsfraktion; $E S V i=$ endsystolisches Volumen geindext; $f R V=$ funktioneller rechter Ventrikel; LA = linker Vorhof; LV = linker Ventrikel; $Q p=$ Lungenperfusion; $Q s$ = Systemperfusion; RA = rechter Vorhof; SD = Standardabweichung; TK = Trikuspidalklappe.

Fett gedruckt: $p<0,05$ (signifikante Ergebnisse).

In den MRT-Untersuchungen von 2013 und von 2016 ergaben sich für das Kollektiv insgesamt ähnliche Werte für die Herzfunktionsparameter, allerdings war ein signifikanter Unterschied in der fRV-EF nachzuweisen. Diese hatte sich von 2013 bis 2016 verringert. 
Die Messungen der Volumina der einzelnen Herzkammern ergaben einige signifikante Unterschiede zu den Voruntersuchungen. Insgesamt fanden sich in den MRTUntersuchungen von 2016 vergrößerte fRV-EDVi bei 12 von 20 Patienten (60\%). Bei einer Patientin wurde ein verkleinertes LV-EDVi ermittelt, alle anderen Patienten hatten normwertige Volumina des LV (Normwerte gemäß Alfakih et al. 2003 und Sarikouch et al. 2010).

Rechte Herzseite: Das EDVi sowie das ESVi des fRV vergrößerten sich signifikant (siehe Tabelle 3). Auch das ESVi des RA nahm signifikant an Größe zu, während das EDVi das RA nicht signifikant variierte. Die Volumina des rechten Herzens wurden demnach im Vergleich zu den Voruntersuchungen von 2013 anhand der neuen MRT-Datensets vergrößert gemessen. Mit einer Änderung von im Mittel $15 \mathrm{ml} / \mathrm{m}^{2}$ im ESVi und $13 \mathrm{ml} / \mathrm{m}^{2}$ im EDVi des fRV ist hier ein deutlicher Unterschied erkennbar. Unverändert blieben die endsystolischen sowie enddiastolischen Volumina das aRV.

Linke Herzseite: Das EDVi sowie das ESVi des LV vergrößerten sich signifikant. Ebenso nahm das ESVi des LA signifikant an Größe zu. Die Werte liegen laut Milliliterangaben allerdings sehr nah an den Vorwerten. Bei signifikanten $p$-Werten bei den linksventrikulären und linksatrialen Volumenangaben schwanken diese im Mittel um maximal 4 $\mathrm{ml} / \mathrm{m}^{2}$. Demnach ist von einer leichten Zunahme der Volumina der linken Herzkammern auszugehen. Unverändert blieb das EDVi des LA.

\subsection{Der Totale Rechts/Links-Volumen-Index 2013 und 2016}

Die folgende Tabelle (Tabelle 4) zeigt die Werte des Totalen Rechts/Links-VolumenIndex aller Studienteilnehmer der Jahre 2013 und 2016 im Vergleich sowie die relative Änderung des Index-Wertes in Prozent (\%). 
Tabelle 4: Der Totale Rechts/Links-Volumen-Index aller Studienteilnehmer 2013 und $2016 \mathrm{im}$ Vergleich.

\begin{tabular}{|c|c|c|c|}
\hline Patient & R/L-VI 2013 & R/L-VI 2016 & Relative Änderung (\%) \\
\hline 1 & 2,5 & 2,6 & 3,4 \\
\hline 2 & 1,2 & 1,3 & 5,8 \\
\hline 3 & 1,9 & 1,9 & $-1,2$ \\
\hline 4 & 2,0 & 2,1 & 4,1 \\
\hline 5 & 2,6 & 2,6 & $-1,0$ \\
\hline 6 & 1,9 & 1,8 & $-4,1$ \\
\hline 7 & 2,3 & 2,2 & $-4,7$ \\
\hline 8 & 1,2 & 1,2 & $-3,0$ \\
\hline 9 & 2,2 & $*$ & \\
\hline 10 & 2,9 & 2,7 & $-5,8$ \\
\hline 11 & 3,4 & 3,6 & 6,0 \\
\hline 12 & 2,4 & 2,5 & 3,7 \\
\hline 13 & $x$ & 2,9 & \\
\hline 14 & 1,3 & $*$ & \\
\hline 15 & 1,6 & $*$ & \\
\hline 16 & 2,1 & 2,1 & $-3,8$ \\
\hline 17 & 2,9 & 2,9 & $-1,7$ \\
\hline 18 & 1,9 & 2,0 & 3,1 \\
\hline 19 & 3,3 & 3,2 & $-0,8$ \\
\hline 20 & 1,5 & $x$ & \\
\hline 21 & 1,3 & 1,3 & 2,7 \\
\hline 22 & 6,9 & 6,7 & $-2,2$ \\
\hline 23 & 1,2 & 1,3 & 6,1 \\
\hline 24 & 3,9 & 4,2 & 7,7 \\
\hline 25 & 3,3 & 3,1 & $-6,0$ \\
\hline 26 & 7,7 & + & \\
\hline
\end{tabular}

* = lost in follow-up; $\mathrm{X}=$ keine MRT-Untersuchung; + = verstorben;

Änderungen $>5 \%$ werden in fettgedruckter Schrift angegeben. 
Aus den Daten der Tabelle 4 wird ersichtlich, dass alle Patienten mit EA Index-Werte von > 1,2 haben, da das rechte Herzenvolumen gegenüber dem Volumen des linken Herzens bei der EA vergrößert ist. Die Werte des R/L-VI reichten im Jahr 2013 von einem Indexwert von minimal 1,2 bis zum höchsten Wert von 7,7. Im Jahr 2016 lag der niedrigste Wert ebenso bei 1,2, während der höchste errechnete Wert 6,7 war. Der Patient mit dem höchsten Indexwert von 2013 war vor den Follow-Up-Untersuchungen verstorben. Die Werte schwanken im Vergleich zu den Voruntersuchungen im Mittel um 3,8\%. Wie anhand der Vorzeichen der relativen Änderung sichtbar ist, wurden die Volumina und somit der Index teilweise vergrößert und teilweise verkleinert gemessen. Die größte Differenz eines Indexwertes liegt bei $0,3(3,9 \rightarrow 4,2)$, die relative Änderung liegt hier bei 7,7\% und ist somit die größte Änderung im gesamten Kollektiv. Die folgende Tabelle (Tabelle 5) zeigt die Gesamtergebnisse der Berechnung des R/LVolumen-Index anhand der bildgebenden Verfahren von 2013 und 2016:

Tabelle 5: Anzahl, Mittelwert und Standardabweichung des Totalen Rechts/Links-VolumenIndex 2013 und 2016.

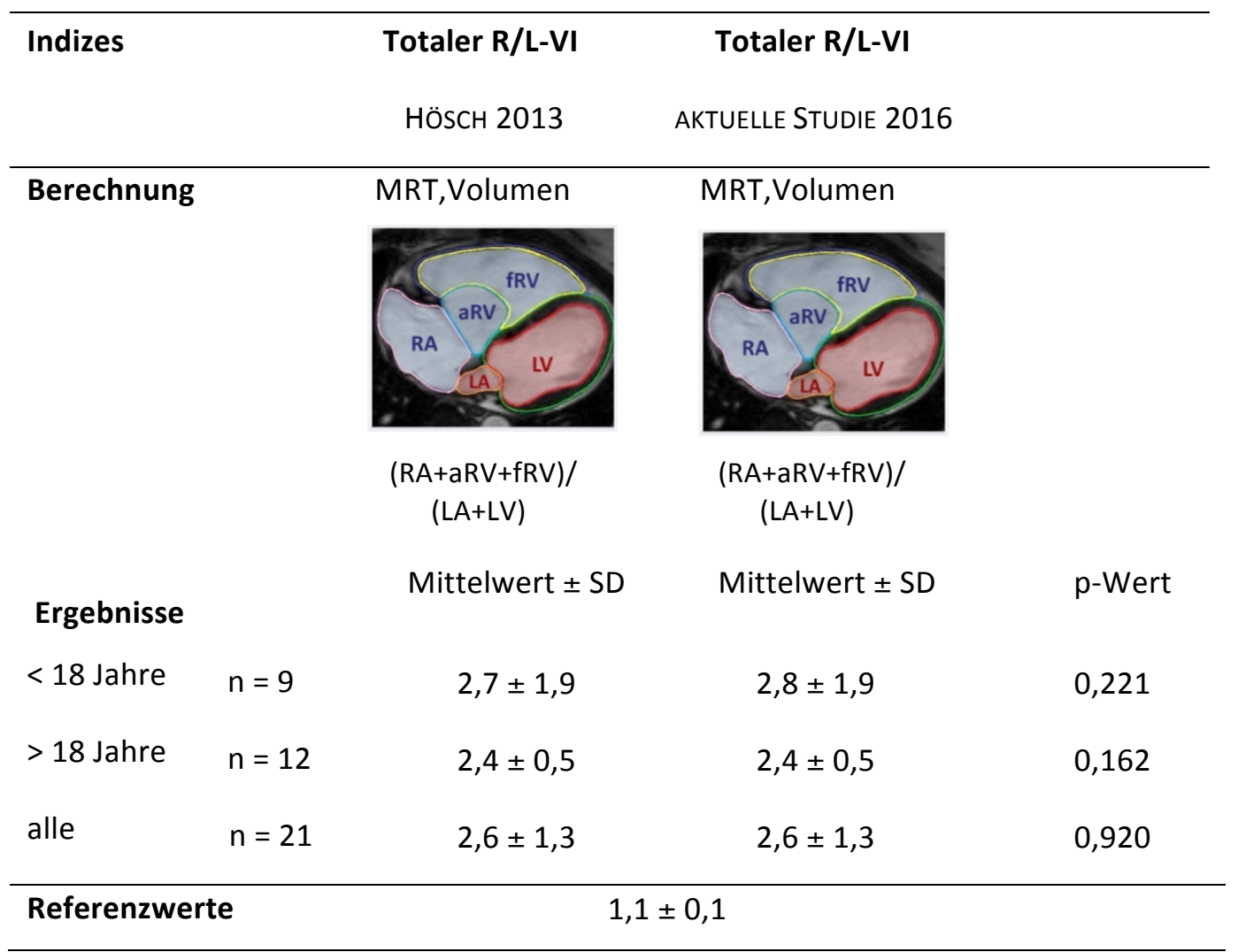

$\mathrm{aRV}=$ atrialisierter rechter Ventrikel; $\mathrm{fRV}=$ funktioneller rechter Ventrikel; $L A=$ linker Vorhof; $\mathrm{LV}=$ linker Ventrikel; RA = rechter Vorhof; SD = Standardabweichung. 
Anhand dieser Zahlen wird ersichtlich, dass sich der Totale R/L-VI in den letzten drei Jahren in dem Studienkollektiv im Mittel kaum verändert hat. Die Untersuchungen ergaben bei den beiden Messungen für das gesamte Kollektiv den gleichen Mittelwert von 2,6 mit einer Standardabweichung von $\pm 1,3$. Bei der Aufteilung in unter 18-jährige und über 18-jährige Patienten liegt der Mittelwert für minderjährige Patienten bei 2,7 \pm 1,9 im Jahre 2013 bzw. bei 2,8 \pm 1,9 im Jahr 2016, während der R/L-VI bei volljährigen Patienten konstant bei 2,4 $\pm 0,5$ liegt. Demnach liegt der Mittelwert des Index in der jüngeren Altersgruppe etwas über dem der älteren Patienten, der Indexwert nimmt im Laufe von drei Jahren im minderjährigen Kollektiv tendenziell etwas zu. Die T-Tests für den R/L-VI im Vergleich von 2013 zu 2016 liegen aber mit p-Werten von 0,920 für das Gesamtkollektiv; 0,221 für unter 18-jährige Patienten und 0,162 für volljährige Patienten allesamt über 0,05 und gelten somit als nicht signifikant.

Die folgende Tabelle (Tabelle 6) zeigt die Ergebnisse der Korrelation nach Pearson des Totalen R/L-VI mit den klinischen Parametern 2013 und 2016.

Anhand der Daten aus Tabelle 6 wird deutlich, dass der R/L-VI mit den Messwerten für die klinischen Parameter je nach Jahr und dementsprechend abweichendem Patientenkollektiv unterschiedlich stark korreliert. Ein Korrelationskoeffizient $(r)$ von $>0,4$ bzw. $<-0,4$ und ein entsprechender $p$-Wert von unter 0,05 gelten als signifikant.

Der R/L-VI zeigte 2013 eine signifikante Korrelation mit den folgenden Parametern: BNP, QRS- und QTc-Dauer, max. $\mathrm{VO}_{2} / \mathrm{kg}$, max. $\mathrm{O}_{2}$-Puls, VE/VCO 2 , TAPSE und echokardiographischer Schweregrad der TI (Hösch 2015).

In den Untersuchungen von 2016 korreliert der Index weiterhin mit den Parametern QRS-Dauer, max. $\mathrm{VO}_{2} / \mathrm{kg}$ und $\mathrm{VE} / \mathrm{VCO}_{2}$. 
Tabelle 6: Pearson-Korrelationen des Totalen Rechts/Links-Volumen-Index mit klinischen Parametern 2013 und 2016.

\begin{tabular}{|c|c|c|}
\hline Index & $\begin{array}{c}\text { Totaler R/L-VI } \\
2013\end{array}$ & $\begin{array}{c}\text { Totaler R/L-VI } \\
2016\end{array}$ \\
\hline Klinische Daten & $\mathrm{n}=25$ & $\mathrm{n}=21$ \\
\hline NYHA* & $r=0,401 ; p=0,052$ & $r=0,426 ; p=0,061$ \\
\hline BNP & $r=0,691 ; p=0,0003$ & $r=0,132 ; p=0,580$ \\
\hline QRS-Dauer & $r=0,432 ; p=0,039$ & $r=0,484 ; p=0,026$ \\
\hline QTc-Dauer & $r=0,465 ; p=0,029$ & $r=0,268 ; p=0,240$ \\
\hline
\end{tabular}

\section{Spiroergometrie}

$\begin{array}{lll}\text { Max. } \mathrm{VO}_{2}(\%) & r=-0,479 ; p=0,024 & r=-0,518 ; p=0,019 \\ \text { VCO }_{2}(\%) & r=0,426 ; p=0,048 & r=0,639 ; p=0,002 \\ & r=-0,532 ; p=0,011 & r=-0,326 ; p=0,161\end{array}$

\section{Echokardiographie}

TI visuell*

$$
r=0,557 ; p=0,005 \quad r=-0,038 ; p=0,873
$$

TAPSE

$$
r=0,554 ; p=0,006 \quad r=0,220 ; p=0,380
$$

*Spearman Rank

$\mathrm{BNP}=$ brain natriuretic peptide; Max. = Maximale; NYHA = New York Heart Association; $r=$ Pearsons Korrelationskoeffizient; TAPSE = tricuspid annular plane systolic excursion; $\mathrm{TI}=$ Trikuspidalklappeninsuffizienz; $\mathrm{VE} / \mathrm{VCO}_{\mathbf{2}}=$ exspiratorischer $\mathrm{CO}_{2}$-Quotient; $\mathrm{VO}_{2}=$ maximale Sauerstoffaufnahme.

Fett gedruckt: $p<0,05$ (signifikante Ergebnisse). 
Es folgt die graphische Darstellung der Pearson-Korrelationen des Totalen R/L-VI mit statistisch signifikanten klinischen Parametern von 2013 und 2016 im Vergleich (Abbildung 6 - Abbildung 10); $p$ und $r$ Werte sind der Tabelle 6 zu entnehmen.

\section{A}

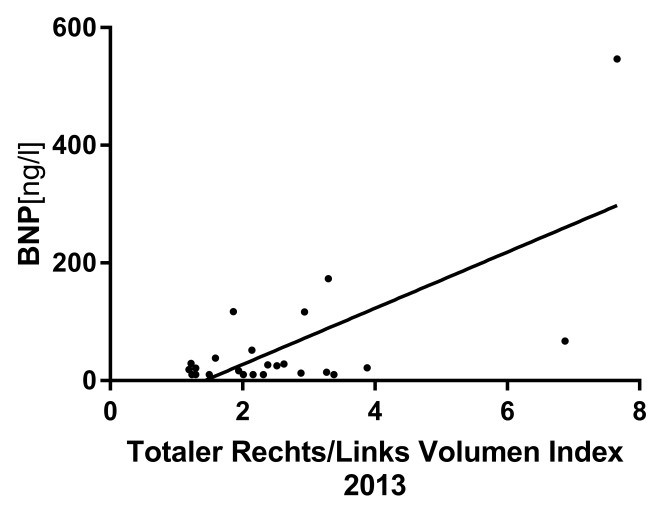

6. B

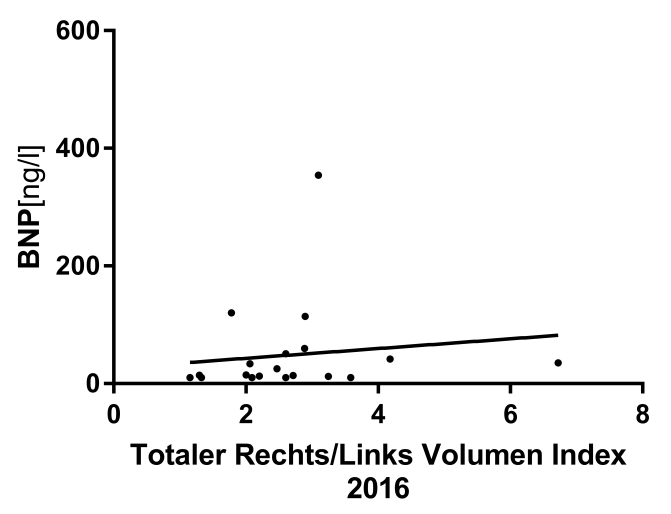

Abbildung 6: Ergebnisse der Pearson-Korrelationen des laborchemischen Parameters BNPWert mit dem Totalen Rechts/Links-Volumen-Index aus dem Jahr 2013 und aus dem Jahr 2016. $\mathrm{BNP}=$ brain natriuretic peptide

7. A

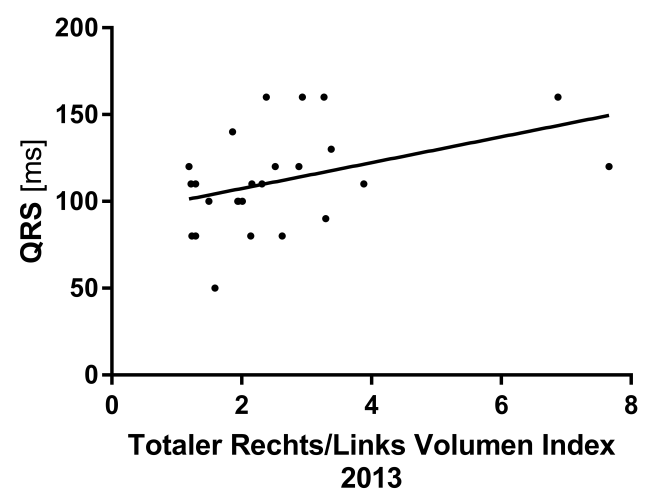

7. B

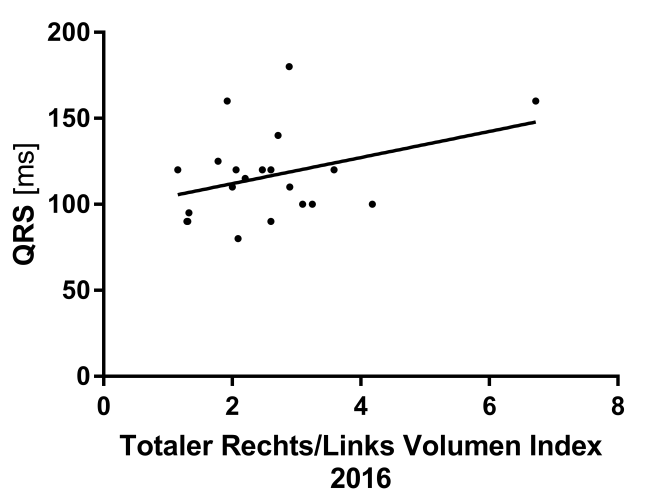

Abbildung 7: Ergebnisse der Pearson-Korrelationen des EKG-Parameters QRS-Zeit mit dem Totalen Rechts/Links-Volumen-Index aus dem Jahr 2013 und aus dem Jahr 2016. 
8. A

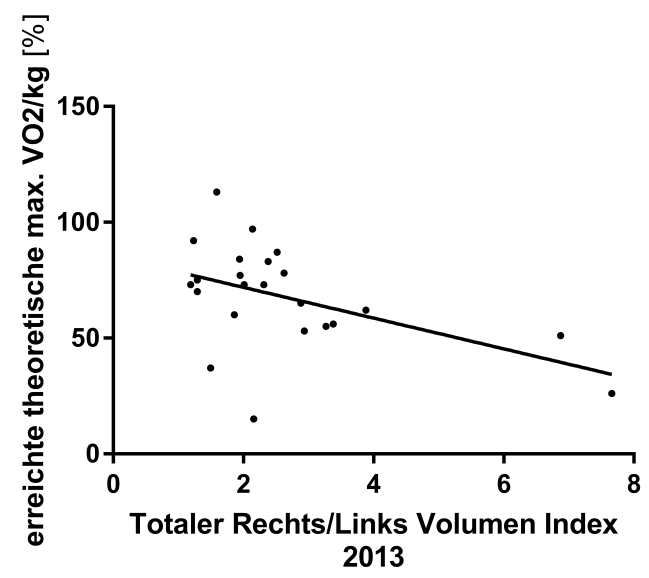

8. B

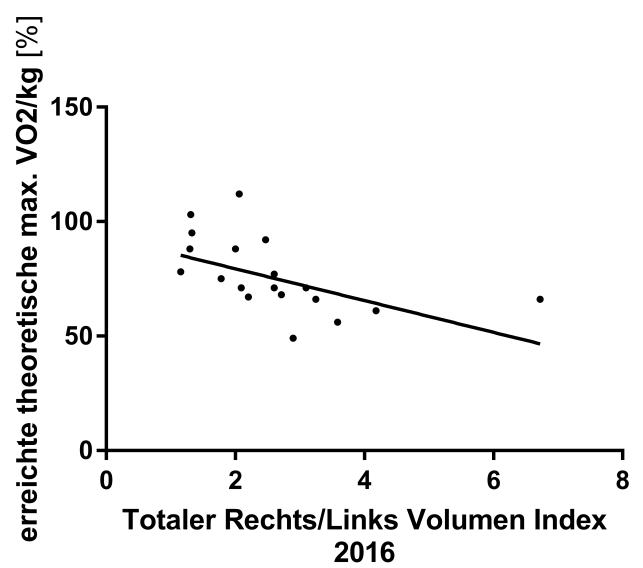

Abbildung 8: Ergebnisse der Korrelationen des spiroergometrischen Parameters max. $\mathrm{VO}_{2} / \mathrm{kg}$ mit dem Totalen Rechts/Links-Volumen-Index aus dem Jahr 2013 und aus dem Jahr 2016.

Max. $\mathrm{VO}_{2} / \mathrm{kg}=$ Maximale Sauerstoffaufnahme.

9. A

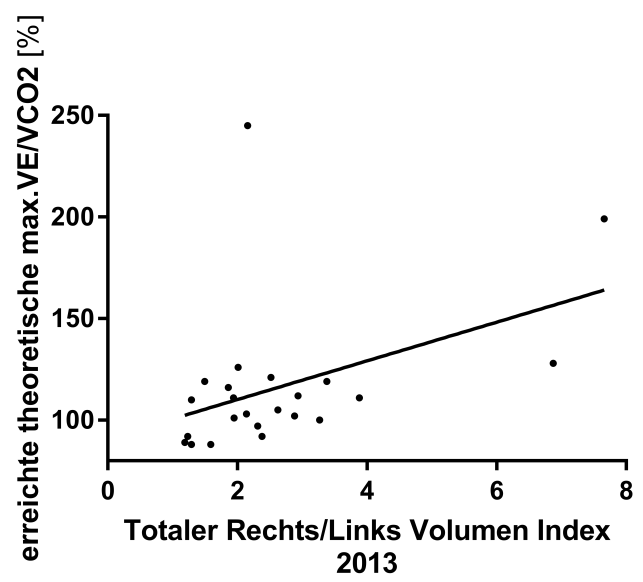

9. B

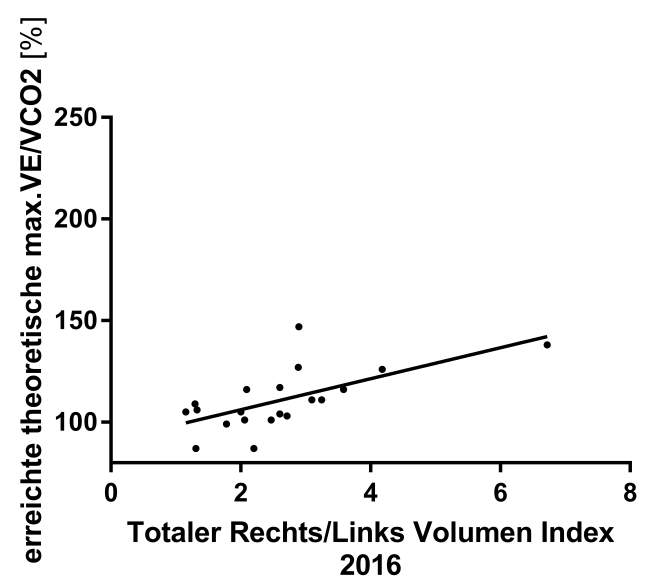

Abbildung 9: Ergebnisse der Pearson-Korrelationen des spiroergometrischen Parameters max. $\mathrm{VE} / \mathrm{VCO}_{2}$ mit dem Totalen Rechts/Links-Volumen-Index aus dem Jahr 2013 und aus dem Jahr 2016.

Max. VE/ $/ \mathrm{VCO}_{2}=$ Exspiratorischer $\mathrm{CO}_{2}$-Quotient. 
10. A

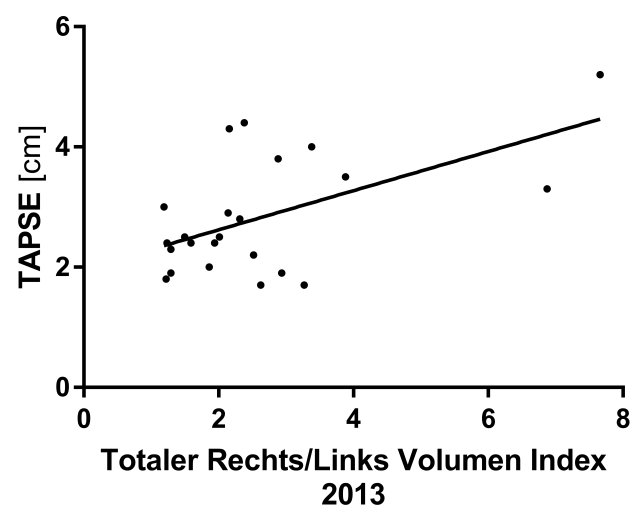

10. B

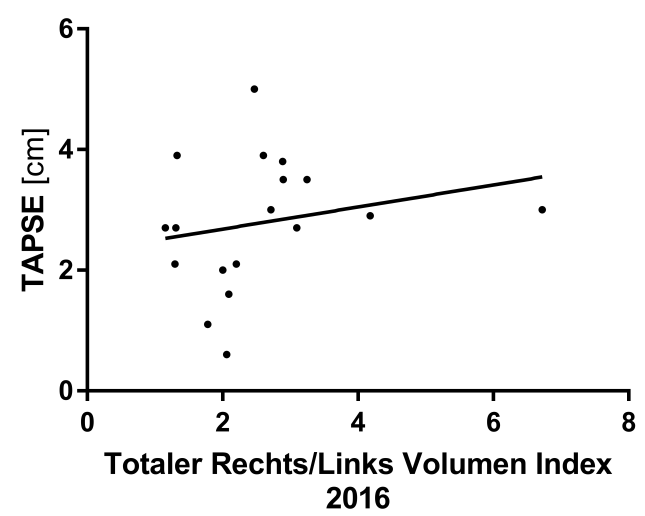

Abbildung 10: Ergebnisse der Pearson-Korrelationen des echokardiographischen Parameters TAPSE mit dem Totalen Rechts/Links-Volumen-Index aus dem Jahr 2013 und aus dem Jahr 2016.

TAPSE = tricuspid annular plane systolic excursion.

\subsection{Der Totale Rechts/Links-Volumen-Index als prognostischer Parameter zur Risikostratifizierung für das Auftreten kardialer Ereignisse}

Die folgende Tabelle (Tabelle 7) dient dem Vergleich der R/L-VI der Patienten, bei denen innerhalb von drei Jahren kardiale Ereignisse auftraten mit jenen, die den bisherigen Beobachtungszeitraum ereignisfrei überlebten. Als kardiale Ereignisse werden in dieser Studie Tod durch Herzversagen, das Auftreten von Tachykardien während des Beobachtungszeitraumes und eine Verschlechterung der NYHA-Klasse gewertet.

Tabelle 7: Vergleich der Totalen Rechts/Links-Volumen-Indizes der Patienten mit und ohne aufgetretene kardiale Ereignisse innerhalb von drei Jahren.

Totaler R/L-VI 2016

\begin{tabular}{lll}
\hline Studienkohorte $(\mathbf{n}=\mathbf{2 2})$ & & \\
& Median & Mittelwert \pm SD \\
Patienten mit Events & 3,1 & $3,9 \pm 2,2$ \\
Patienten ohne Events & 2,2 & $2,5 \pm 1,3$ \\
\hline
\end{tabular}

$\mathrm{SD}=$ standard deviation 
Innerhalb der letzten drei Jahre sind insgesamt fünf Ereignisse aufgetreten, davon ein Todesfall und drei klinisch relevante Tachykardien. Bei zwei Patienten ging das Auftreten von Tachykardien mit einer subjektiv verminderten körperlichen Belastbarkeit einher, sodass sie in der ersten Folgeuntersuchung in eine höhere NYHA-Klasse eingestuft wurden. Ein weiterer Patient wurde ohne das Auftreten von Tachykardien aufgrund verminderter körperlicher Belastbarkeit ebenfalls in eine höhere NYHA-Klasse eingestuft.

Deutlich wird, dass der R/L-VI bei Patienten mit kardialem Ereignis in den letzten drei Jahren bei 3,9 \pm 2,2 (Mittelwert \pm Standardabweichung) liegt, während ereignisfreie Patienten einen niedrigeren R/L-VI von im Mittel 2,5 $\pm 1,3$ haben.

Um den Nutzen des Totalen R/L-VI zur Risikoberechnung für das Eintreten kardialer Ereignisse zu evaluieren, wurde für verschiedene Schweregrade der EA eine KaplanMeier-Kurve erstellt. Ein R/L-VI von unter 2,0 (Gruppe I) gilt als niedriger Schweregrad der EA, während ein R/L-VI von über 2,0 (Gruppe II) als hoher Schweregrad und somit als Risikofaktor angesehen wird.

Das Kaplan-Meier-Verfahren wird hier angewandt, um das Risiko zu berechnen, mit dem bei einem Patienten mit einem Risikofaktor ( $R / L-V I>2,0)$ innerhalb eines bestimmten Zeitraumes von drei Jahren ein kardiales Ereignis eintritt. Mit Hilfe der Kaplan-Meier-Kurve lassen sich somit Raten für ein ereignisfreies Überleben bestimmen (Kaplan \& Meier 1985). In unserer Studie zeigen die Raten, bei wie vielen Patienten bis zum Ende des Beobachtungszeitpunktes von derzeit drei Jahren kein kardiales Ereignis eingetreten ist. 
Die folgende Abbildung (Abbildung 11) zeigt die Ergebisse des Kaplan-Meier-Schätzers:

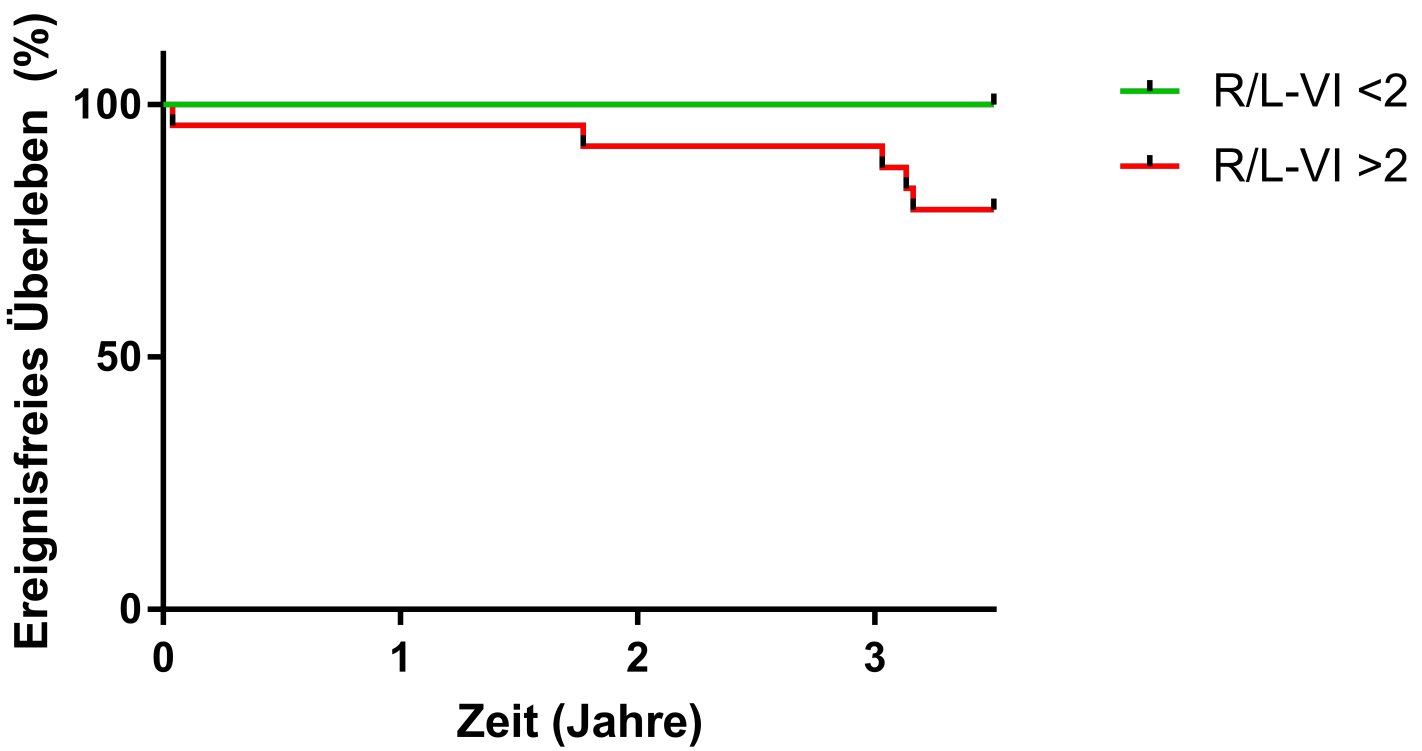

Abbildung 11: Ergebnisse der Überlebenszeitanalyse nach Kaplan-Maier.

Vergleich der Patientengruppen I und II mit einem Totalen Rechts/Links-Volumen-Index von $<2,0$ und $>2,0$.

$\mathrm{R} / \mathrm{L}-\mathrm{VI}=$ Rechts/Links-Volumen-Index.

Die folgende Tabelle (Tabelle 8) zeigt die Anzahl an Patienten mit und ohne aufgetretene kardiale Ereignisse für die Gruppe I mit einem $\mathrm{R} / \mathrm{L}-\mathrm{VI}$ von $<2,0$ und für die Gruppe II mit einem R/L-VI von > 2,0 in einem Zeitraum von drei Jahren.

Tabelle 8: Vergleich der Anzahl an Patienten mit und ohne aufgetretene kardiale Ereignisse in drei Jahren.

\begin{tabular}{cll}
\hline Kardiologische Ereignisse & Patienten mit R/L-VI $<2$ & Patienten mit R/L-VI $>2$ \\
\hline Todesfall & $0(0 \%)$ & $1(0,5 \%)$ \\
Tachykardien & $0(0 \%)$ & $3(14 \%)$ \\
NYHA-Verschlechterung & $0(0 \%)$ & $3(14 \%)$ \\
\hline
\end{tabular}

NYHA $=$ New York Heart Association. 
Die Rate an ereignisfreiem Überleben nach drei Jahren beträgt für die Gruppe I mit einem R/L-VI von < 2,0 100\% und für die Gruppe II mit einem R/L-VI von > 2,0 79,2\%. Demnach wird erwartet, dass innerhalb von drei Jahren bei $79,2 \%$ der Patienten mit einem R/L-VI von > 2,0 kein kardiales Ereignis eintritt. Demgegenüber erwartet man bei $0 \%$ der Patienten mit einem R/L-VI von $<2,0$ das Eintreten eines kardialen Ereignisses innerhalb von drei Jahren. Der p-Wert zum Vergleich der zwei Gruppen beträgt 0,037. Zum Signifikanzniveau $\alpha=5 \%$ ist der Unterschied zwischen den beiden Gruppen signifikant.

Das HR wurde ebenfalls berechnet und dient dem Vergleich der zwei Patientengruppen (Ressing et al. 2010). Betrachtet man die Zeit bis zum Auftreten eines kardialen Ereignisses so ist das Risiko für das Eintreten des Ereignisses (das Hazard) höher für die Gruppe II mit einem R/L-VI von über 2,0 als für die Gruppe I mit einem R/L-VI unter 2,0. In unserer Studie beträgt das HR für Patienten mit einem R/L-VI von über 2,0 im Vergleich zu Patienten mit einem R/L-VI von <2,0 sogar 6,472 (CI: 1,115-37,56). Das relative Risiko für das Eintreten eines kardialen Ereignisses ist für Patienten mit einem R/LVI von $>2,0$ also 6,5-mal so hoch wie für Patienten mit einem $R / L-V I$ von $<2,0$.

Patienten aus der Gruppe mit einem R/L-VI von $<2,0$ leben demnach länger ohne das Eintreten eines kardialen Ereignisses, als Patienten mit einem R/L-VI von über 2,0.

\subsection{Reproduzierbarkeit}

Zur Berechnung der Reproduzierbarkeit wurde der R/L-VI aus der Addition der Einzelvolumina ermittelt. In der Bland-Altman-Analyse für die Intraobserver-Variabilität wurden der systematische Fehler („Verzerrung“, engl. bias), die Standardabweichung des systematischen Fehlers und die 95\% Konfidenzintervalle (engl. Confidence Interval, $\mathrm{Cl}$ ) berechnet. Die Ergebnisse der Intraobserver- und Interobservertests für den R/L-VI mittels Bland-Altman-Analyse fielen mit einem Intraobserver-bias von $0,046 \mathrm{ml} / \mathrm{m}^{2}$ $(95 \% \mathrm{Cl}:-0,237-0,329)$ und einem Interobserver-bias von $-0,005 \mathrm{ml} / \mathrm{m}^{2}(95 \% \mathrm{Cl}$ : $0,177-0,167)$ extrem gering aus. 


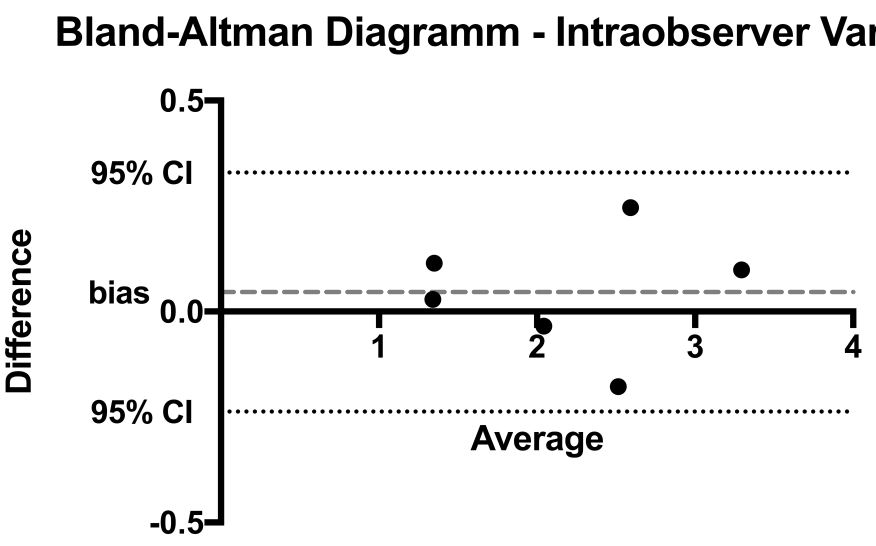

Abbildung 12: Bland-Altman-Diagramm für die Intraobserver-Variabilität des Totalen Rechts/Links-Volumen-Index.

$\mathrm{Cl}=$ confidence interval.

\section{Bland-Altman Diagramm - Interobserver Variabilität}

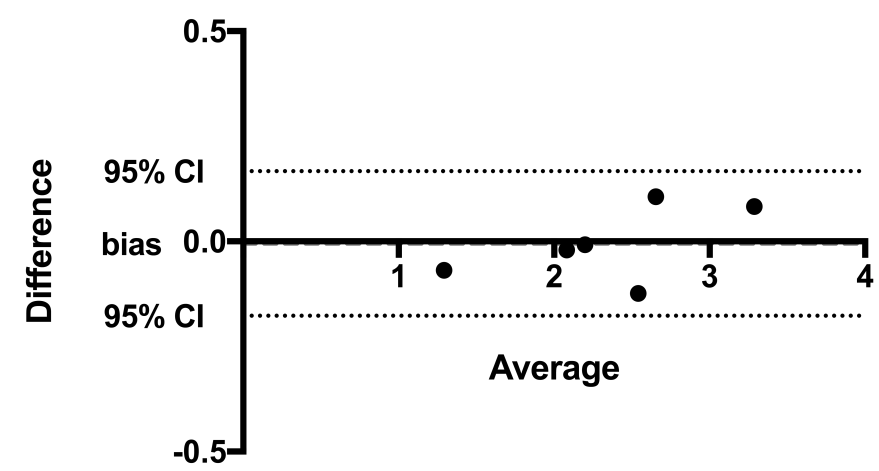

Abbildung 13: Bland-Altman-Diagramm für die Interobserver-Variabilität des Totalen Rechts/Links-Volumen-Index.

$\mathrm{Cl}=$ confidence interval .

Der ICC für die Intraobserver-Variabilität des R/L-VI lag bei 0,992 (95\% Cl: 0,949-0,999). Der ICC für die Interobserver-Variabilität erzielte Werte von 0,997 ( $95 \% \mathrm{Cl}$ : 0,978 - 1,0). Der CoV-Wert des R/L-VI lag bei den Intraobservertests im Mittel bei 3,8\% (range 1,04\% - 6,8\%), der CoV-Wert für die Interobserver-Untersuchungen bei 2,0\% (range 0,26\% - 3,8\%) (siehe Tabelle 9 und Tabelle 10). 
Für die vereinfachte Berechnung des Index wurden lediglich die Gesamtvolumina der rechten und linken Herzkammern bestimmt.

Um zu überprüfen, ob diese einfachere Art und Weise des manuellen Einzeichenens unterschiedliche Werte für den R/L-VI ergäbe, wurde auch für die vereinfachte Methode zur Ermittlung des R/L-VI durch Umfahren der Gesamtkontur der rechten und linken Herzhälfte im Vergleich mit der aufwendigeren Methode zur Errechnung des R/L-VI aus Einzelkammervolumina die Reproduzierbarkeit bei 19 Patienten getestet. Der bias lag laut der Bland-Altman-Analyse bei 0,004 ml/ $\mathrm{m}^{2}(95 \% \mathrm{Cl}-0,043-0,051)$, der CoV lag bei 1,8\% und der ICC lag bei 0,997 (95\% Cl 0,991-0,999). Das Ergebnis zeigt einen nur minimalen Unterschied zu der Berechnung des Index anhand der Segmentierung aller Herzkammern getrennt voneinaner. Demnach kann der R/L-VI vereinfacht errechnet werden aus dem Gesamtvolumen des rechten Herzens (RHV) geteilt durch das gesamte Linksherzvolumen (LHV): R/L-VI = RHV/LHV.

Die folgende Abbildung (Abbildung 14) zeigt das Bland-Altman-Diagramm für die Errechnung des Totalen Rechts/Links-Volumen-Index mittels Einzelkammersegmentierung im Vergleich zur Errechnung mittels Umfahren der Gesamtkonturen der rechten und linken Herzhälfte.

\section{Bland-Altman Diagramm Einzelkammersegmentierung vs. Gesamtkonturen}

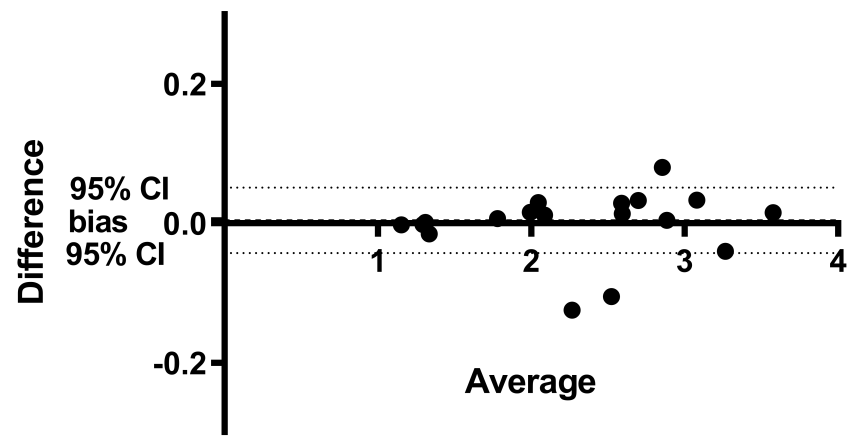

Abbildung 14: Bland-Altman-Diagramm für die Errechnung des Totalen Rechts/Links-VolumenIndex mittels Einzelkammersegmentierung im Vergleich zur Errechnung mittels Gesamtkontureneinzeichnung der rechten und linken Herzhälfte.

$\mathrm{Cl}=$ confidence interval. 
Die Reproduzierbarkeit der Einzalkammervolumina wurde mittels Variationskoeffizienten berechnet.

Die Ergebnisse des Variationskoeffizientens (engl. Coefficient of Variation, CoV in \%) für die Intra- und Interobserver-Variabilitäten sind in Tabelle 9 zusammengefasst:

Tabelle 9: Variationskoeffizient (CoV) für die Intraobserver- und die Interobserver-Variabilität 2016.

\begin{tabular}{|c|c|c|c|}
\hline \multicolumn{2}{|c|}{ Studienkohorte $(n=6)$} & $\begin{array}{l}\text { Intraobserver } \\
\text { CoV (\%) }\end{array}$ & $\begin{array}{l}\text { Interobserver } \\
\text { CoV (\%) }\end{array}$ \\
\hline Totaler R/L-VI & & 3,8 & 2,0 \\
\hline \multicolumn{4}{|c|}{ Einzelkammervolumina } \\
\hline \multirow[t]{8}{*}{ Rechtes Herz } & RA EDV & 4,3 & 4,0 \\
\hline & RA ESV & 7,2 & 13,6 \\
\hline & aRV EDV & 10,3 & 15,5 \\
\hline & aRV ESV & 19,0 & 21,8 \\
\hline & fRV EDV & 3,7 & 8,6 \\
\hline & fRV ESV & 9,8 & 14,3 \\
\hline & fRV EF (\%) & 10,9 & 9,7 \\
\hline & Qp & 10,9 & 12,9 \\
\hline \multirow[t]{6}{*}{ Linkes Herz } & LA EDV & 11,0 & 3,6 \\
\hline & LA ESV & 11,9 & 10,2 \\
\hline & LV EDV & 2,6 & 5,4 \\
\hline & LV ESV & 2,4 & 6,5 \\
\hline & LV EF & 2,3 & 2,9 \\
\hline & Qs & 3,5 & 8,1 \\
\hline
\end{tabular}

$\mathrm{aRV}=$ atrialisierter rechter Ventrikel; CoV = coefficient of variation; EDV = enddiastolisches Volumen; $E F$ = Ejektionsfraktion; ESV = endsystolisches Volumen; $f R V=$ funktioneller rechter Ventrikel; LA = linkes Atrium; LV = linker Ventrikel; $Q p=$ Lungenperfusion; Qs = Systemperfusion; $\mathrm{RA}=$ rechtes Atrium.

In Tabelle 9 sind die Intra- und Interobserver-Variabilitäten anhand des CoV dargestellt. Die kleinsten CoV-Werte liegen bei 2,4\% für das ESV des LV im Intraobserver- und bei 
3,6\% für das EDV des LA im Interobservertest. Die größten Werte für den CoV liegen bei 19,0 \% für das ESV des aRV im Intraobserver- und bei $21,8 \%$ ebenfalls für das ESV des aRV im Interobservertest.

Der R/L-VI ergab einen sehr niedrigen CoV-Wert von nur 3,8\% im Intraobserver- und 2,0 im Interobservertest.

Der Intraklassen-Korrelationskoeffizient (ICC) wurde ebenfalls berechnet. Die Ergebnisse des ICC für Intraobserver-Variabilitäten sind in Tabelle 10 ersichtlich:

Tabelle 10: Ergebnisse für die Intraobserver-Variabilität 2016; Der IntraklassenKorrelationskoeffizient (ICC).

\begin{tabular}{|c|c|c|c|}
\hline \multicolumn{2}{|c|}{ Studienkohorte $(n=6)$} & \multirow{2}{*}{$\begin{array}{l}\text { ICC } \\
0,992\end{array}$} & \multirow{2}{*}{$\begin{array}{l}95 \% \mathrm{Cl} \\
0,949-0,999\end{array}$} \\
\hline Totaler R/L-VI & & & \\
\hline \multicolumn{4}{|c|}{ Einzelkammervolumina } \\
\hline \multirow[t]{8}{*}{ Rechtes Herz } & RA EDV (ml) & 0,992 & $0,929-0,999$ \\
\hline & RA ESV (ml) & 0,983 & $0,897-0,998$ \\
\hline & aRV EDV (ml) & 0,988 & $0,921-0,998$ \\
\hline & aRV ESV (ml) & 0,980 & $0,871-0,997$ \\
\hline & fRV EDV (ml) & 0,995 & $0,967-0,999$ \\
\hline & fRV ESV (ml) & 0,972 & $0,773-0,996$ \\
\hline & fRV EF (\%) & 0,770 & $-0,175-0,966$ \\
\hline & Qp (I/min) & 0,760 & $0,482-0,966$ \\
\hline \multirow[t]{6}{*}{ Linkes Herz } & LA EDV (ml) & 0,812 & $0,152-0,973$ \\
\hline & LA ESV (ml) & 0,826 & $0,314-0,976$ \\
\hline & LV EDV (ml) & 0,980 & $0,855-0,997$ \\
\hline & LV ESV (ml) & 0,987 & $0,907-0,998$ \\
\hline & LV EF (\%) & 0,941 & $0,551-0,992$ \\
\hline & Qs (I/min) & 0,954 & $0,652-0,994$ \\
\hline
\end{tabular}

$\mathrm{aRV}=$ atrialisierter rechter Ventrikel; $\mathrm{Cl}=$ confidence interval; $\mathrm{EDV}=$ enddiastolisches Volumen; $\mathrm{EF}=$ Ejektionsfraktion; $\mathrm{ESV}=$ endsystolisches Volumen; $\mathrm{fRV}=$ funktioneller rechter Ventrikel; $\mathrm{ICC}=$ intraclass correlation coefficient; $\mathrm{LA}=$ linkes Atrium; $\mathrm{LV}=$ linker Ventrikel; $\mathrm{Qp}=$ Lungenperfusion; Qs = Systemperfusion; RA = rechtes Atrium. 
Die Ergebnisse des ICC im Intraobservertest fielen für die Volumina sehr gut aus, der ICC nahm durchgehend hohe Werte an. Diese reichten von 0,812 für das EDV des LA bis zu 0,995 für das EDV des fRV. Das LA erzielte die schlechtesten Werte der Volumina im Intraobservertest, während das EDV des fRV die höchsten Werte erzielte. Der Mittelwert des ICC lag insgesamt bei 0,855. Die fRV-EF erzielte mit 0,770 generell die schlechtesten Werte im Intraobservertest. Demgegenüber erreichte der R/L-VI einen sehr hohen ICC Wert von 0,992. Die Ergebnisse des ICC für Interobserver-Variabilitäten sind in Tabelle 11 zusammengefasst:

Tabelle 11: Ergebnisse für die Interobserver-Variabilität 2016; Der IntraklassenKorrelationskoeffizient (ICC).

\begin{tabular}{|c|c|c|c|}
\hline \multicolumn{2}{|c|}{ Studienkohorte $(n=6)$} & ICC & $95 \% \mathrm{Cl}$ \\
\hline \multicolumn{2}{|l|}{ Totaler R/L-VI } & 0,997 & $0,978-1,0$ \\
\hline \multicolumn{4}{|c|}{ Einzelkammervolumina } \\
\hline \multirow[t]{8}{*}{ Rechtes Herz } & RA EDV (ml) & 0,986 & $0,905-0,998$ \\
\hline & RA ESV (ml) & 0,782 & $-0,407-0,969$ \\
\hline & aRV EDV (ml) & 0,875 & $0,254-0,982$ \\
\hline & aRV ESV (ml) & 0,954 & $0,699-0,993$ \\
\hline & fRV EDV (ml) & 0,822 & $-0,022-0,974$ \\
\hline & fRV ESV (ml) & 0,384 & $-3,791-0,915$ \\
\hline & fRV EF (\%) & 0,818 & $-0,104-0,974$ \\
\hline & Qp (I/min) & 0,779 & $0,491-0,969$ \\
\hline \multirow[t]{6}{*}{ Linkes Herz } & LA EDV (ml) & 0,982 & $0,869-0,997$ \\
\hline & LA ESV (ml) & 0,072 & $-23,108-0,863$ \\
\hline & LV EDV (ml) & 0,911 & $0,420-0,987$ \\
\hline & LV ESV (ml) & 0,993 & $0,957-0,999$ \\
\hline & LV EF (\%) & 0,951 & $0,682-0,993$ \\
\hline & Qs (I/min) & 0,720 & $-0,303-0,958$ \\
\hline
\end{tabular}

$\mathrm{ICC}=$ intraclass correlation coefficient; $\mathrm{Cl}=$ confidence interval; $\mathrm{RA}=$ rechtes Atrium; $\mathrm{aRV}=$ atrialisierter rechter Ventrikel; fRV = funktioneller rechter Ventrikel; $E F=$ Ejektionsfraktion; $Q p$ = Lungenperfusion; LA = linkes Atrium LV = linker Ventrikel; Qs = Systemperfusion; EDV = enddiastolisches Volumen; ESV = endsystolisches Volumen. 
Die Ergebnisse des ICC im Interobservertest fielen insgesamt ebenfalls gut aus. Der ICC nahm Werte von 0,072 für das ESV des LA bis zu 0,993 für das ESV des LV an. Der LA erzielte die schlechtesten Werte der Volumina im Interobservertest, die fRV-EF erzielte mit einem ICC von 0,818 generell schlechtere Werte als die LV-EF im Interobservertest. Der ICC Wert für den R/L-VI übertraf mit 0,997 alle Ergebnisse für die Einzelkammervolumina.

Eine Bland-Altman-Analyse wurde ebenfalls durchgeführt. Tabelle 12 zeigt die BlandAltman-Analyse für die Intraobserver-Variabilität 2016:

Tabelle 12: Ergebnisse der Bland-Altman-Analyse für die Intraobserver-Variabilität 2016.

\begin{tabular}{|c|c|c|c|c|}
\hline Studienkohorte $(n=6)$ & & Bias & SD of bias & $95 \% \mathrm{Cl}$ \\
\hline Totaler R/L-VI & & 0,046 & 0,13 & $0,24-0,33$ \\
\hline \multicolumn{5}{|l|}{ Einzelkammervolumina } \\
\hline \multirow[t]{8}{*}{ Rechtes Herz } & RA EDVi $\left(\mathrm{ml} / \mathrm{m}^{2}\right)$ & $-4,2$ & 5,4 & $-14,9-6,4$ \\
\hline & RA ESVi $\left(\mathrm{ml} / \mathrm{m}^{2}\right)$ & $-2,0$ & 4,3 & $-10,3-6,3$ \\
\hline & aRV EDVi $\left(\mathrm{ml} / \mathrm{m}^{2}\right)$ & $-1,0$ & 6,4 & $-13,5-11,5$ \\
\hline & aRV ESVi $\left(\mathrm{ml} / \mathrm{m}^{2}\right)$ & $-1,2$ & 5,9 & $-12,8-10,3$ \\
\hline & fRV EDVi $\left(\mathrm{ml} / \mathrm{m}^{2}\right)$ & 2,6 & 5,4 & $-8,1-13,2$ \\
\hline & fRV ESVi $\left(\mathrm{ml} / \mathrm{m}^{2}\right)$ & 6,2 & 8,1 & $-9,6-22,0$ \\
\hline & fRV EF (\%) & $-4,75$ & 6,12 & $-16,7-7,24$ \\
\hline & Qp (I/min) & $-0,2$ & 0,5 & $-1,3-0,8$ \\
\hline \multirow[t]{6}{*}{ Linkes Herz } & LA EDVi $\left(\mathrm{ml} / \mathrm{m}^{2}\right)$ & $-4,9$ & 4,9 & $-14,5-4,6$ \\
\hline & LA ESVi $\left(\mathrm{ml} / \mathrm{m}^{2}\right)$ & $-1,0$ & 4,1 & $-8,8-7,1$ \\
\hline & LV EDVi $\left(\mathrm{ml} / \mathrm{m}^{2}\right)$ & $-0,2$ & 3,6 & $-7,1-6,8$ \\
\hline & LV ESVi $\left(\mathrm{ml} / \mathrm{m}^{2}\right)$ & $-0,1$ & 1,5 & $-3,0-2,8$ \\
\hline & LV EF (\%) & 0,13 & 2,38 & $-4,5-4,8$ \\
\hline & Qs (I/min) & $<0,1$ & 0,2 & $-0,4-0,4$ \\
\hline
\end{tabular}

aRV = atrialisierter rechter Ventrikel; $\mathrm{Cl}=$ confidence interval; $\mathrm{EDVi}=$ enddiastolisches Volumen geindext; $\mathrm{EF}=$ Ejektionsfraktion; $\mathrm{ESVi}=$ endsystolisches Volumen geindext; $f R V=$ funktioneller rechter Ventrikel; $L A=$ linkes Atrium; $L V=$ linker Ventrikel; $Q p=$ Lungenperfusion; $Q s=$ Systemperfusion; RA = rechtes Atrium; SD = standard deviation. 
In der Bland-Altman-Analyse für die Intraobserver-Variabilität reichten die Werte für die Einzelkammervolumina vom kleinsten bias mit einem Wert von $-0,1 \mathrm{ml} / \mathrm{m}^{2}$ für das EDV des LV bis zum größten bias mit einem Wert von $6,2 \mathrm{ml} / \mathrm{m}^{2}$ für das ESV des fRV. Die fRV-EF erzielte im Intraobservertest einen bias von -4,75, die LV-EF einen bias von 0,13 . Der Totale R/L-VI zeigt im Vergleich zu den Messungen der einzelnen Volumina einen minimalen bias von 0,046.

Die folgende Tabelle (Tabelle 13) zeigt die Ergebnisse der Bland-Altman-Analyse für die Interobserver-Variabilität 2016:

Tabelle 13: Ergebnisse der Bland-Altman-Analyse für die Interobserver-Variabilität 2016.

\begin{tabular}{|c|c|c|c|c|}
\hline Studienkohorte $(n=6)$ & & Bias & SD of bias & $95 \% \mathrm{Cl}$ \\
\hline Totaler R/L-VI & & $-0,005$ & 0,09 & $-0,17-0,16$ \\
\hline \multicolumn{5}{|l|}{ Einzelkammervolumina } \\
\hline \multirow[t]{8}{*}{ Rechtes Herz } & RA EDVi $\left(\mathrm{ml} / \mathrm{m}^{2}\right)$ & 4,0 & 5,7 & $-7,3-15,3$ \\
\hline & RA ESVi $\left(\mathrm{ml} / \mathrm{m}^{2}\right)$ & $-4,3$ & 13,2 & $-30,2-21,6$ \\
\hline & aRV EDVi $\left(\mathrm{ml} / \mathrm{m}^{2}\right)$ & 10,1 & 15,5 & $-20,3-40,5$ \\
\hline & aRV ESVi $\left(\mathrm{ml} / \mathrm{m}^{2}\right)$ & 5,2 & 8,6 & $-11,6-22,0$ \\
\hline & fRV EDVi $\left(\mathrm{ml} / \mathrm{m}^{2}\right)$ & $-15,2$ & 19,0 & $-52,5-22,1$ \\
\hline & $f R V \operatorname{ESVi}\left(\mathrm{ml} / \mathrm{m}^{2}\right)$ & $-13,1$ & 11,5 & $-35,6-9,4$ \\
\hline & fRV EF (\%) & $-5,0 \%$ & $-6,0$ & $-10,0-(-2,0)$ \\
\hline & Qp (I/min) & $-0,3$ & 0,8 & $-1,9-1,3$ \\
\hline \multirow[t]{6}{*}{ Linkes Herz } & LA EDVi $\left(\mathrm{ml} / \mathrm{m}^{2}\right)$ & 0,2 & 1,9 & $-3,4-3,9$ \\
\hline & LA ESVi $\left(\mathrm{ml} / \mathrm{m}^{2}\right)$ & 1,2 & $-4,1$ & $-6,9-9,2$ \\
\hline & LV EDVi $\left(\mathrm{ml} / \mathrm{m}^{2}\right)$ & $-3,6$ & 5,5 & $-14,2-7,2$ \\
\hline & LV ESVi $\left(\mathrm{ml} / \mathrm{m}^{2}\right)$ & $-0,9$ & 3,3 & $-7,3-5,6$ \\
\hline & LV EF (\%) & $-0,1$ & $-5,0$ & $-2,0-3,0$ \\
\hline & Qs (I/min) & $-0,3$ & 0,3 & $-0,9-0,3$ \\
\hline
\end{tabular}

$\mathrm{aRV}=$ atrialisierter rechter Ventrikel; $\mathrm{Cl}=$ confidence interval; $\mathrm{EDVi}=$ enddiastolisches Volumen geindext; $\mathrm{EF}=$ Ejektionsfraktion; $\mathrm{ESVi}=$ endsystolisches Volumen geindext; $\mathrm{fRV}=$ funktioneller rechter Ventrikel; $L A=$ linkes Atrium; $L V=$ linker Ventrikel; $Q p=$ Lungenperfusion; $Q s=$ Systemperfusion; RA = rechtes Atrium; SD = standard deviation. 
Die Werte der Bland-Altman-Analyse für die Interobserver-Variabilität reichten vom kleinsten bias mit einem Wert von $0,2 \mathrm{ml} / \mathrm{m}^{2}$ für das EDV des LA bis zum größten bias mit einem Wert von $-15,2 \mathrm{ml} / \mathrm{m}^{2}$ für das EDV des fRV. Die LV-EF erzielte einen bias von $-0,1 \%$, die fRV-EF einen bias von $5 \%$. Der Totale R/L-VI zeigt im Vergleich zu den Messungen der einzelnen Volumina einen minimalen bias von 0,005.

\subsection{Regressionsanalyse}

Um den Zusammenhang einer möglichen Veränderung des $\mathrm{R} / \mathrm{L}-\mathrm{VI}$ und einer Veränderung der klinischen Parameter zu untersuchen, wurde eine Regressionsanalyse durchgeführt. Bei den Parametern mit den höchsten Pearson-Korrelationswerten ergaben sich die in der folgenden Tabelle (Tabelle 14) aufgeführten Ergebnisse in der Regressionsanalyse.

Tabelle 14: Ergebnisse der multiplen Regressionsanalyse.

\begin{tabular}{|c|c|c|c|}
\hline & $R, R^{2}, b, b^{*}$ & Adjusted $\mathrm{R}^{2}$ & p-Wert \\
\hline \multicolumn{4}{|c|}{ Klinische Parameter } \\
\hline \multirow[t]{2}{*}{ BNP } & $R=0,513 ; R^{2}=0,264$ & \multirow[t]{2}{*}{0,172} & \multirow[t]{2}{*}{0,840} \\
\hline & $b=0,004 ; b^{*}=0,045$ & & \\
\hline \multirow[t]{2}{*}{ QRS-Dauer } & $R=0,541 ; R^{2}=0,292$ & \multirow[t]{2}{*}{0,202} & \multirow[t]{2}{*}{0,022} \\
\hline & $b=-0,149 ; b^{*}=-0,652$ & & \\
\hline \multirow[t]{2}{*}{$\mathrm{Max} . \mathrm{VO}_{2}$} & $R=0,249 ; R^{2}=0,062$ & \multirow{2}{*}{0,464} & \multirow{2}{*}{0,496} \\
\hline & $b=-0,058 ; b^{*}=-0,183$ & & \\
\hline \multirow[t]{2}{*}{$\mathrm{VE} / \mathrm{VCO}_{2}(\%)$} & $R=0,044 ; R^{2}=0,209$ & \multirow{2}{*}{0,322} & \multirow{2}{*}{0,739} \\
\hline & $b=0,042 ; b^{*}=0,092$ & & \\
\hline
\end{tabular}

$a \cdot R^{2}=$ adjustiertes $R^{2} ; b=$ Regressionskoeffizient; $b^{*}=$ standartisierter Regressionskoeffizient; $\mathrm{BNP}=$ brain natriuretic peptide; $\max . \mathrm{VO}_{2}=$ maximale Sauerstoffaufnahme; $\mathrm{R}=$ multipler Korrelationskoeffizient; $\mathrm{R}^{2}=$ Bestimmtheitsmaß; $\mathrm{VE} / \mathrm{VCO}_{2}=$ exspiratorischer $\mathrm{CO}_{2}$-Quotient.

Fett gedruckt: $p<0,2$ (signifikante Ergebnisse). 
In dieser Studie wurde eine multiple Regressionanalyse angewandt, welche die abhängige Variable durch mehrere unabhängige Variablen erklärt. In der Analyse war die relative Veränderung des R/L-VI (Dreijahreswert von 2016 minus Ausgangswert von 2013 geteilt durch den Ausgangswert) die Einflussgröße (unabhängige, erklärende Variable), die relative Veränderung der anderen Parameter (z.B. des BNP-Werts) die Zielgröße (abhängige Variable) und der Ausgangswert der Zielvariablen (z.B. BNP-Wert von 2013) die Kovariable.

Die Regressionsanalyse der Änderung des R/L-VI im Bezug auf die Änderung der QRSDauer gilt als signifikant, da der $p$-Wert einen Wert von $<0,2$ annimmt. Hierbei liegt der Wert des Regressionskoeffizientens b bei -0,149, während der standartisierte Regressionskoeffizient $b^{*}-0,652$ ergibt. Wenn der R/L-VI um eine Einheit größer würde, nähme die QRS-Dauer also voraussichtlich leicht ab. Allerdings liegt das Bestimmtheitsmaß $\mathrm{R}^{2}$ laut der Analyse bei nur 0,292 (29\%) bei der Betrachtung des Einflusses einer Änderung im R/L-VI auf eine Änderung des QRS-Wertes. Das korrigierte Bestimmtheitsmaß (adjustiertes $R^{2}=a \cdot R^{2}$ ) liegt laut der Analyse sogar nur bei 0,204 (20\%). Das Modell ist somit unzureichend angepasst.

Die übrigen untersuchten Parameter nehmen nicht signifikante $p$-Werte von $>0,2$ an. Weiterhin wurde der Einfluss von den Parametern Alter und Geschlecht auf die Veränderung des R/L-VI (abhängige Variable) untersucht. Hierbei zeigte sich kein signifikanter Einfluss ( $p$-Wert $>0,2)$ bei dem Parameter Geschlecht $(p=0,817)$. Der Parameter Alter zeigte mit einem p-Wert von 0,029 einen signifikanten Einfluss auf den R/L-VI. Der Wert für den Regressionskoeffizienten $b$ war allerdings mit $-0,001\left(b^{*}:-0,501\right)$ sehr niedrig und negativ, somit spräche ein zunehmendes Alter der Patienten für eine Verringerung des $\mathrm{R} / \mathrm{L}-\mathrm{VI}$. 


\section{Diskussion}

\subsection{Zusammenfassung der Haupthypothesen}

Durch die im Rahmen dieser Dissertation durchgeführte erste Folgeuntersuchung einer prospektiven Studie wird der vor drei Jahren eingeführte R/L-VI zur Schweregradeinteilung der EA evaluiert und validiert. Die Ergebnisse der Untersuchungen sowie die Aussagekraft und die Anwendbarkeit des Index in der Praxis werden im Folgenden diskutiert. Dabei wird ein besonderes Augenmerk auf die folgenden Fragen gelegt:

Ist es im Verlauf von drei Jahren zu einer signifikanten Veränderung des R/L-VI bei dem Studienkollektiv gekommen? Ist der R/L-VI als bildgebungsbasierter Parameter für die Schweregradeinteilung der EA geeignet? Besteht ein Zusammenhang zwischen Veränderungen des klinischen Zustandes der Patienten und einer Veränderung des R/L-VI? Treten kardiale Eregnisse insbesondere bei Patienten mit einem hohen R/L-VI von > 2,0 auf?

Zusammenfassend ist somit verbindendes Thema der Diskussion, ob der R/L-VI der Risikoberechnung für kardiale Ereignisse und als Vorhersageparameter für das klinische Outcome dienen und somit zur Verlaufskontrolle und als Entscheidungshilfe für Therapiemaßnahmen im klinischen Alltag eingesetzt werden kann.

\subsection{Veränderungen des Totalen Rechts/Links-Volumen-Index im Bezug auf den klinischen Zustand der Patienten}

Der Totale R/L-VI war in der Nachuntersuchung bei allen 21 Patienten fast unverändert messbar. Der Index zeigt damit bisher konsistente Werte und ist über den ersten Beobachtungszeitraum dieser prospektiven Studie als cMRT-Parameter stabil.

Auch die Änderungen in den messbaren klinischen Parametern waren im Kollektiv gering. Der größte Anteil der Patienten war körperlich unverändert gut belastbar und gesund. Abgesehen von Alter, Größe, Gewicht, BMI und BSA, die im Rahmen des Wachstums- bzw. Alterungsprozesses des Kollektivs erklärbar sind, zeigen sich weder in den laborchemischen noch in den apparativen Untersuchungen signifikante Unterschiede zu den Voruntersuchungen von 2013. 
Die subjektive Leistungsfähigkeit, die in dieser Studie anhand der NYHA-Klassifikation eingeschätzt wird, verschlechterte sich allerdings bei drei Patienten bereits in den ersten drei Jahren des Beobachtungszeitraumes. Die NYHA-Klassifikation dient als simple Klassifizierungsmethode für eine verminderte Leistungsfähigkeit basierend auf subjektiven Symptomen und wird heute auch zunehmend bei Patienten mit angeborenen Herzfehlern eingesetzt (Diller et al. 2005).

Die Ergebnisse der NYHA-Klassifikation als subjektive Herzinsuffizienzmarker korrelierten in unserem Kollektiv jedoch weder im Jahr 2013 noch im Jahr 2016 mit dem R/L-VI als objektivem Schweregradparameter der Erkrankung (Hösch 2015).

Eine Ursache hierfür könnte die Subjektivität der NYHA-Einteilung sein. Die Klassifikation scheint den wahren Grad der Limitierung der körperlichen Leistungsfähigkeit bei Patienten mit angeborenen Herzfehlern zu unterschätzen (Diller et al. 2005). Ursächlich hierfür könnte sein, dass sich Patienten mit angeborenen Herzfehlern bereits ihr Leben lang an ihre Herzerkrankung und deren langsames Fortschreiten angepasst haben, sodass sie sich in ihrer körperlichen Belastung unbewusst beschränken. Daher sind sie sich des wahren Ausmaßes ihrer eingeschränkten Leistungsfähigkeit nicht unbedingt bewusst und geben eine „normale Belastbarkeit" trotz objektiv messbarer Einschränkung an (Diller et al. 2005). Eine „normale physische Aktivität“ ist demnach schwer definierbar bei Patienten, deren Leistungsfähigkeit schon immer limitiert war (Dimopoulos et al. 2006).

Laut Rostagno et al. (2000) ist die Reproduzierbarkeit der NYHA-Klassifikation generell niedrig, da ihr subjektive Symptome zugrunde liegen. Da diverse Einflussgrößen wie persönlicher Trainingszustand, Übergewicht oder Sportunlust etc. in die Einteilung einfließen, ist eine Hochstufung in der NYHA-Klasse nicht unbedingt auf eine Größenzunahme des rechten Herzens zurückzuführen. Aus diesem Grund sind die Veränderungen der NYHA-Klassifikation in unserem Kollektiv nicht anhand von Veränderungen in den cMRT-Parametern im Sinne einer Vergrößerung des R/L-VI abbildbar.

Demgegenüber zeigt der R/L-VI weiterhin eine gute Korrelation mit dem mittels 12 Kanal-EKG gemessenen Parameter QRS-Zeit. 
Eine Verlängerung der QRS-Zeit zeigt eine verlängerte Erregungsausbreitung im Ventrikelmyokard des Herzens an und kann ein Marker für eine Vergrößerung des RV und eine RV-Dysfunktion bei EA sein (Assenza et al. 2013). Der terminale Anteil des QRSKomplexes bildet die Aktivierung des RV ab. Eine verzögerte Depolarisation und eine abnormale Aktivierung des RV sind häufige Befunde bei der EA (Assenza et al. 2013). Sung et al. (1975) zeigten, dass eine verlängerte QRS-Zeit bei Patienten mit ASD nicht aufgrund eines Rechtschenkelblocks, sondern aufgrund einer RV-Dilatation entsteht. Brown et al. (2008) wiesen nach, dass bei Patienten mit EA eine verlängerte QRS-Zeit mit einer erhöhten Mortalität im Langzeit-Follow-Up assoziiert ist (Brown et al. 2008). Assenza et al. fanden im Jahr 2013 heraus, dass eine verlängerte QRS-Zeit bei Patienten mit EA für eine Funktionseinschränkung des Herzens spricht, da die QRS-Zeit mit einem vergrößerten RV-EDV und invers mit der RV-EF korreliert. Patienten mit EA mit einer verlängerten QRS-Zeit zeigten in der Studie generell ein schlechteres klinisches Profil. Demnach identifiziert eine verlängerte QRS-Zeit eine Risikogruppe unter den Patienten mit EA mit einem größeren aRV, einer höheren NYHA-Klasse, einer niedrigeren RV-EF und einer verminderten körperlichen Belastbarkeit auf dem Spiroergometer gemessen an dem Parameter $\mathrm{VO}_{2} / \mathrm{kg}$. Diese Patienten zeigen voraussichtlich einen schlechteren Verlauf der Erkrankung, sodass eine intensivere klinische Überwachung erforderlich ist (Assenza et al. 2013).

Die Ergebnisse der vorgelegten eigenen Studie sind in Einklang mit voherigen Erkenntnissen zur QRS-Breite. Da der R/L-VI auch in der Folgestudie von 2016 weiterhin eine gute Korrelation mit der QRS-Zeit zeigt und beide Parameter eine Vergrößerung des RV bzw. des rechten Herzens nachweisen, ist davon auszugehen, dass der R/L-VI ein valides Maß für die kardiale Belastung bei EA ist.

Außerdem ist auffällig, dass der R/L-VI weiterhin gute Korrelationen mit den spiroergometrischen Belastungsparametern maximale Sauerstoffaufnahme (max. $\mathrm{VO}_{2}$ ) und dem $\mathrm{CO}_{2}$-Atemäquivalent $\left(\mathrm{VE} / \mathrm{VCO}_{2}\right)$ zeigt. Neben der Funktion als Marker für eingeschränkte körperliche Belastbarkeit werden diese bei Erwachsenen mit angeborenen Herzfehlern als Prädiktoren für einen ungünstigen Krankheitsverlauf angesehen (Diller et al. 2005; Dimopoulos et al. 2006). Im Gegensatz zur NYHA-Klassifikation ist die maximale Sauerstoffaufnahme eine kontinuierliche Variable, die eine objektive und re- 
produzierbare Methode zur Einschätzung der körperlichen Fitness repräsentiert (Koike et al. 1992).

Bei Patienten mit angeborenen Herzfehlern $(n=727)$ und Patienten mit EA $(n=32)$ wiesen Diller et al. (2006) nach, dass niedriegere Werte für die maximale Sauerstoffaufnahme und die Herzfrequenzreserve mit einer erhöhten Mortalität assoziiert sind. Eine Studie von Dimopoulos et al. aus dem Jahr 2006 bestätigt die Bedeutung des $\mathrm{CO}_{2}$ Atemäquivalentes sowie der maximalen Sauerstoffaufnahme als Prädiktoren für eine erhöhte Mortalität bei 560 Patienten mit verschiedenen angeborenen Herzfehlern, unter ihnen 20 Patienten mit EA. Eine Studie von Trojnarska et al. aus dem Jahr 2006 stellt heraus, dass sich mit Zunahme des Schweregrades der EA $(n=21)$ die körperliche Belastbarkeit objektiviert durch die spiroergometrische Messgröße max. $\mathrm{VO}_{2} / \mathrm{kg}$ verringert.

Eine reduzierte körperliche Belastbarkeit im Sinne einer erniedrigten maximalen Sauerstoffaufnahme und Herzfrequenzreserve wird auch von anderen Autoren auf einen hohen Schweregrad der Erkrankung zurückgeführt und als ein starker unabhängiger Prädiktor für ein negatives Outcome beschrieben (Radojevic et al. 2013).

Allerdings zeigten die bisher im klinischen Alltag eingesetzten Klassifikationen nach Celermajer et al. (1992) und Carpentier et al. (1988) keine Korrelation mit spiroergometrischen Größen wie der maximalen $\mathrm{VO}_{2} / \mathrm{kg}$ und dem $\mathrm{VE} / \mathrm{VCO}_{2}$ (Tobler et al. 2013; Trojnarska et al. 2006; Hösch et al. 2014). Im Gegensatz zu diesen Klassifikationen korreliert der R/L-VI sowohl im Jahr 2013 als auch im Jahr 2016 mit den Messgrößen für körperliche Leistungsfähigkeit der Spiroergometrie. Somit zeigt sich der R/L-VI auch in dieser Hinsicht als Parameter des Schweregrades der EA, welcher konsistent mit der objektivierbaren Leistungsfähigkeit der Patienten mit EA in der Spiroergometrie korreliert, was seine Stärke als validem Bildgebungsparameter unterstreicht.

Als weiterer objektiver Marker für Herzinsuffizienz wurde in anderen Studien der Plasma-BNP-Wert bei Patienten mit EA erhoben und ein Zusammenhang mit dem Ausprägungsgrad der Erkrankung untersucht. 
In Trojnarskas Studie korrelierte der BNP-Wert im Gegensatz zu den spiroergometrischen Messgrößen allerdings nicht signifikant mit dem Schweregrad der EA nach echokardiographischer Einteilung (Trojnarska et al. 2006).

In der vorliegenden Studie erreichte der Plasma-BNP-Wert laut der statistischen Korrelation nach Pearson mit dem R/L-VI zwar anhand der Untersuchungsergebnisse von 2013 ein Signifikanzniveau von $<0,05$, die Korrelation der beiden Parameter fiel im Jahr 2016 jedoch nicht mehr signifikant aus. Aufgrund der Ergebnisse aus dem Jahr 2013 nahm unsere Arbeitsgruppe an, dass Patienten mit einem höheren Schweregrad der EA auch ein höheres BNP-Level zeigen (Hösch et al. 2015b). Dies ist bei den Untersuchungen im Rahmen des ersten Follow-Ups allerdings nicht bestätigt worden.

Eine mögliche Begründung für die nun nicht mehr signifikanten Ergebnisse der Korrelationen ist der Tod und damit Entfall der Daten des schwerstkranken Patienten des Kollektivs nach 2013. Der Patient zeigte im Jahr 2013 stärkste Herzinsuffizienzsymptome und es ließ sich ein extrem hoher BNP-Wert von $546,5 \mathrm{ng} / \mathrm{l}$ in seinem Plasma nachweisen. Er wurde als einziger aller Studienteilnehmer in die NYHA-Klasse IV eingestuft. Auch in den körperlichen, spiroergometrischen und echokardiographischen Untersuchungen fielen seine Werte am deutlichsten negativ aus. Der Patient litt an einer extrem ausgeprägten Form der EA und sein R/L-VI erreichte einen Wert von 7,7. Sowohl der sehr hohe Indexwert als auch die schlechten Werte in allen weiteren klinisch messbaren Parametern fehlten im Jahr 2016 für die Nachfolgeuntersuchungen. Dies könnte erklären, weswegen die Korrelation in dieser Folgestudie im Bezug auf den BNP-Wert nicht signifikant ausfiel.

Zusammenfassend ist herauszustellen, dass die NYHA-Klassifikation als subjektiver Marker für die Herzinsuffizienz schlecht mit dem objektiven R/L-VI korreliert, während die QRS-Zeit und die spiroergometrischen Untersuchungswerte als objektive Maße einer nachweisbar verringerten körperlichen Leistungsfähigkeit weiterhin gut mit dem R/L-VI korrelieren. Der Plasma-BNP-Wert zeigt als Herzinsuffizienzparameter im Vergleich dazu erst bei einer weit fortgeschrittenen Herzinsuffizienz mit Volumenbelastung des Herzens steigende Werte, ein pathologisches BNP ist erst bei einem sehr hohen Schweregrad der EA zu erwarten. 
Die regelmäßige Ermittlung des R/L-VI in Zusammenschau mit den etablierten klinischen Parametern aus den EKG- und spiroergometrischen Untersuchungen könnte eine Möglichkeit darstellen, eine Verschlechterung des klinischen Zustandes der Patienten frühzeitig zu erkennen und das Therapiekonzept demnach anzupassen.

\subsection{Beurteilung des Totalen Rechts/Links-Volumen-Index als Ver- laufsparameter und als Parameter zur Risikostratifizierung für das Auftreten kardialer Ereignisse}

Das Wissen über den Krankheitsverlauf und das Langzeit-Outcome bei Patienten mit unkorrigierter EA ist derzeit begrenzt (Attie et. al 2000), eine Progredienz der Krankheit schwer vorhersehbar. Bis heute existiert kein wissenschaftlich ausreichend belegtes diagnostisches Mittel zur Früherkennung einer bevorstehenden Dekompensation der Herzkrankheit. Einen MRT-Parameter zu finden, der als Frühmarker oder „BildgebungsBiomarker" für das Auftreten kardialer Komplikationen eingesetzt werden kann, war aus diesem Grund eines der Ziele der vorgelegten Studie.

Durch regelmäßige cMRT-Untersuchungen der Erkrankten und der Ermittlung des R/LVI könnten bereits frühzeitig Änderungen in den Funktionsparametern und der Herzgröße festgestellt und zur Beurteilung des Krankheitsverlaufs herangezogen werden.

Die cMRT-Untersuchungen der Patienten ergaben hinsichtlich der Herzgröße bereits nach drei Jahren eine geringe Zunahme der linken und eine deutliche Zunahme der rechten Herzkammervolumina. Besonders der fRV hat sich im Kollektiv deutlich vergrößert, gleichzeitig hat sich die EF des fRV signifikant verringert. Die signifikante Änderung des Volumens des fRV deutet darauf hin, dass das rechte Herz bei Patienten mit EA im Laufe der Zeit an Größe zunimmt während die EF des fRV abnimmt. Dies spricht für ein Fortschreiten der Größenzunahme der rechten Herzkammern und eine gleichzeitig abnehmende Auswurfleistung des rechten Herzens im Verlauf der Erkrankung.

Optimal wäre es, mithilfe des R/L-VI eine Progredienz der Krankheit bereits vor dem Auftreten kardialer Ereignisse anzeigen zu können und den R/L-VI als Frühmarker für bevorstehende kardiale Komplikationen nutzen zu können. 
Um das Risiko für Komplikationen einschätzen zu können, wurden bereits verschiedene Studien zur Identifizierung möglicher Prädiktoren für kardiale Ereignisse bei Patienten mit EA durchgeführt.

Eine dieser Studien zur Definition möglicher Prädiktoren für kardiale Komplikationen bei EA wurde von Gentles et al. im Jahr 1992 mit 48 Patienten mit EA über einen Beobachtungszeitraum von 32 Jahren durchgeführt. Signifikante Prädiktoren für einen bevorstehenden Herztod waren das männliche Geschlecht, ein Herz-Thorax-Quotient von > 0,65, NYHA-Klassen III und IV, Atemlosigkeit und das Nicht-Vorhandensein eines WPW-Syndroms (Gentles et al. 1992). Der Herz-Thorax-Quotient wurde hierbei als bester Prädiktor eingestuft (Gentles et al. 1992).

Da der Herz-Thorax Quotient zur Beurteilung der Herzgröße dient, spricht ein Quotient von $>0,65$ für eine Vergrößerung der Herzkammern, die sich im Falle einer Rechtsherzvergrößerung auch im R/L-VI abbildet.

Um weitere Parameter zu definieren, die für einen negativen Verlauf der Herzerkrankung sprechen, beobachtete die Arbeitsgruppe von Attie et al. von 1972 bis 1997 den Krankheitsverlauf bei 72 unoperierten Patienten mit EA (Attie et al. 2000). Prädiktoren für einen bevorstehenden Herztod sind laut ihren Untersuchungen das Alter der Patienten bei Diagnosestellung, das männliche Geschlecht, der Schweregrad der EA (Einteilung in drei Gruppen nach echokardiographischer Beurteilung des Versatzes der TK) und ebenfalls ein Herz-Thorax-Quotient von über 0,65 (Attie et al. 2000). Das Ausmaß der TI, eine Zyanose und die NYHA-Klassifikation wurden von Attie et al. (2000) als signifikante Risikofaktoren identifiziert. Die Ergebnisse von Attie et al. (2000) suggerieren, dass der klinische Verlauf und die Prognose von unoperierten Patienten mit EA von verschiedenen Faktoren beeinflusst werden. Der Schweregrad der EA war nur in Extremfällen der Hauptdeterminant für das Überleben, jedoch nicht in milden bis moderaten anatomischen Ausprägungen, die häufig bei Erwachsenen Patienten mit EA vorzufinden sind (Attie et al. 2000).

Auch in der vorliegenden Studie verstarb der Patient mit dem höchsten R/L-VI von 7,7 und somit der extremsten Ausprägungsform der EA mit 47 Jahren an den Folgen seiner Herzerkrankung. Dieses Ereignis geht mit den oben beschriebenen Beobachtungen von Attie et al. einher. 
In der vorliegenden Studie wurde zur Untersuchung des R/L-VI als Prädiktor für das Eintreten schwerer kardialer Komplikationen das Kaplan-Meier-Verfahren angewendet. Hierbei ist bei Patienten mit einem R/L-VI von > 2,0 das Risiko für das Eintreten eines kardialen Ereignisses 6,5-mal so hoch im Vergleich mit Patienten mit einem R/LVI von $<2,0$.

Bei $24 \%$ der Patienten mit einem R/L-VI von $>2,0$ trat innerhalb von drei Jahren ein kardiales Ereignis ein. Patienten aus der Gruppe mit einem R/L-VI von $<2,0$ hatten kein erhöhtes Risiko für das Eintreten kardialer Ereignisse innerhalb von drei Jahren.

Damit kann ein R/L-VI von $>2,0$ als starker Risikofaktor für das Eintreten kardialer Komplikationen bei Patienten mit EA angesehen werden.

Zur Identifizierung weiterer möglicher Risikofaktoren für den plötzlichen Herztod bei Patienten mit EA wurde aktuell die bisher größte retrospektive Studie ( $n=968$ ) von Attenhofer Jost et al. durchgeführt (Attenhofer Jost et al. 2018).

Ein erhöhtes Risiko für einen plötzlichen Herztod haben laut der Analyse Patienten mit Synkopen, Herzinsuffizienz, einem Hämoglobin-Wert von über $15 \mathrm{~g} / \mathrm{dl}$, koronarer Herzkrankheit, atrialen Arrythmien, ventrikulären Tachykardien in der Vorgeschichte, implantiertem Kardioverter Defibrillator (ICD), einer verlängerten QRS-Dauer, einer Pulmonalstenose und einer Mitralinsuffizienz. Obwohl eine Vergrößerung des RV bei Patienten mit Fallot'scher Tetralogie erwiesene Risikofaktoren sind (Khairy et al. 2014), konnte dieser Parameter in Attenhofer Josts Studie bei Patienten mit EA nicht als signifikanter Prädiktor nachgewiesen werden. Allerdings könnte dies an der eingeschränkten Darstellung der rechten Herzkammern mittels konventioneller 2DEchokardiographie liegen (Attenhofer Jost et al. 2018). Mittels CMRT sind sowohl die Größe als auch die Funktion der rechten Herzkammern adäquater messbar (Surkova et al. 2016), sodass laut Attenhofer Jost et al. (2018) Ergebnisse aus Studien mit cMRTDaten abzuwarten sind.

Eine solche Studie mit cMRT-Daten wurde von der Arbeitsgruppe um Riika Rydman durchgeführt (Rydman et al. 2017). Untersucht wurde die prognostische Vorhersagekraft verschiedener cMRT-Parameter für schwere kardiale Komplikationen bei 79 Patienten mit unkorrigierter EA. Hierzu zählen in der Studie das Neuauftreten persistie- 
render Tachyarrhythmien, das Herzversagen und der plötzliche Herztod (Rydman et al. 2017). Während des Beobachtungszeitraumes von durchschnittlich 3,4 Jahren traten in Rydmans Kollektiv 20 schwere kardiale Komplikationen auf. Bei 17 Patienten traten außerdem erstmalig atriale Tachykardien auf (Rydman et al. 2017).

Untersucht wurde der Zusammenhang zwischen den eingetretenen kardialen Ereignissen und den folgenden cMRT-Parametern: RV-EF, LV-EF, LV-SVi und Herz-Index, sowie der fRV/LV-Index (Tobler et al. 2013) und der Totale R/L-VI (Hösch et al. 2014).

Ein Absinken der LV-EF und der fRV-EF um mehr als 5\% stellten sich als die zwei Parameter mit der stärksten Korrelation heraus. Demgegenüber war die Korrelation des R/L-VI mit schweren kardialen Komplikationen statistisch nicht bedeutsam.

Allerdings stellen Rydman et al. (2017) fest, dass den kardialen Komplikationen sehr häufig atriale Tachykardien vorausgehen, sodass diese als frühe Marker für bevorstehende kardiale Ereignisse gelten. Den atrialen Tachykardien wiederum gingen Veränderungen in den folgenden cMRT Parametern voraus: eine Volumenzunahme der rechten Herzkammern um mehr als $5 \mathrm{ml} / \mathrm{m}^{2}(p=0,005)$, eine Abnahme der RV-EF um mehr als 5\% ( $p=0,011)$, eine Zunahme des fRV/LV-Volumen-Ratios (Tobler et al. 2013) ( $p=$ 0,005), eine Zunahme des Versatzes des apikalen TK-Segels bezogen auf die totale Länge des Ventrikelseptums ( $p=0,041$ ) und eine Zunahme des Totalen R/L-VI (Hösch et al. 2014). Hierbei erreichte die Zunahme des R/L-VI um eine Einheit mit einem p-Wert von 0,002 die besten Korrelationsergebnisse aller in der Studie getesteten cMRTParameter als Prädiktor für atriale Tachykardien (Rydman et al. 2017). Dies bedeutet, dass eine Erhöhung des R/L-VI ein möglicher Prädiktor atrialer Tachykardien ist, welche wiederum häufig schweren kardialen Komplikationen und im schlimmsten Fall dem plötzlichen Herztod vorausgehen.

Unseren Ergebnissen aus dieser prospektiven Studie zufolge kann ein hoher R/L-VI als Risikofaktor für das Auftreten kardialer Ereignisse angesehen werden. Dies ist in Übereinstimmung mit o.g. Daten aus früheren, teils retrospektiven Studien, die z.T. aus dem Thorax-Röntgenbild, der Echokardiographie und dem MRT das Herzvolumen als Parameter heranzogen.

Regelmäßige cMRT-Untersuchungen inklusive der Ermittlung der R/L-VI zur frühzeitigen Feststellung einer Größenzunahme des Herzens sollten deshalb in die Routinekon- 
trollen für Patienten mit EA aufgenommen werden (Rydman et al. 2017). Patienten mit einem ermittelten R/L-VI von > 2,0 sollten gegebenenfalls häufiger als einmal jährlich eine kardiologische Kontrolluntersuchung erhalten. Hierbei sollte ein besonderes Augenmerk auf das Auftreten von Herzrhythmusstörungen und eine Verschlechterung der körperlichen Leistungsfähigkeit gelegt werden.

\subsection{Der Nutzen des Totalen Rechts/Links-Volumen-Index als Pa- rameter für das Treffen therapeutischer Entscheidungen}

Da das Wissen über den Krankheitsverlauf bei Patienten mit unkorrigierter EA derzeit begrenzt ist, werden die Therapieansätze weiterhin kontrovers diskutiert (Attie et. al 2000).

Die EA reicht in ihrem Ausprägungsgrad von minimal bis extrem und dennoch werden sehr unterschiedliche Krankheitsverläufe beschrieben (Moradi et al. 2017). Demnach ist es schwierig, den richtigen Zeitpunkt für eine Intervention abzupassen. Ein MRTParameter, der ein guter Frühmarker für das Auftreten kardialer Komplikationen ist, könnte somit als entscheidender Faktor zur Therapieplanung dienen.

Die Art der Therapie und der Zeitpunkt für ihr Einleiten sind nicht klar definiert (Kawase et al. 2008). Häufig werden Patienten mit EA bereits in jungen Jahren operiert, während andere allein durch konservative Therapiemaßnahmen bis ins hohe Lebensalter asymptomatisch bleiben können. Meist wird eine Operation angeboten, wenn die Patienten von der NYHA-Klasse II in Klasse III oder IV übergehen, eine Zyanose stärker wird oder sich paradoxe Embolien ereignen (Brown et al. 2009). Viele Chirurgen sehen in einer hochgradigen $\mathrm{TI}$ mit moderater rechtsventrikulärer Dysfunktion bereits eine Indikation für eine Operation, sogar bei asymptomatischen Patienten (Kawase et al. 2008). Außerdem wird eine Operation empfohlen, wenn die körperliche Belastbarkeit messbar abnimmt, sich die systolische Herzfunktion verschlechtert, Arrythmien auftreten oder die Herzgröße radiographisch zunimmt bzw. der RV zunehmend dilatiert (Brown et al. 2009).

Für die Beurteilung der rechten Herzgröße ist der R/L-VI ein geeigneter und objektiv messbarer Parameter. Tritt eine Größenzunahme ein, sollte diese mit dem klinischen Zustand der Patienten abgeglichen und in Zusammenschau aller Risikofaktoren mögli- 
che Therapieoptionen mit dem Patienten erörtert werden. Für das Treffen therapeutischer Entscheidungen sollte laut Attenhofer Jost et al. (2018) der klinische Zustand der Patienten, die NYHA-Klasse und die Neigung zu Arrhythmien in Zusammenschau mit cMRT-Markern wie dem Totalen R/L-VI herangezogen werden.

\subsection{Bewertung der Reproduzierbarkeit der MRT-Ergebnisse und des Totalen Rechts/Links-Volumen-Index}

Um klinisch von Nutzen zu sein sollte jede diagnostische Maßnahme den Anspruch erfüllen, akkurat und reproduzierbar zu sein (Geva 2014).

Die cMRT hat sich bei der quantitativen Messung der Herzkammervolumina sowohl in dieser als auch in anderen Studien als akkurat und reproduzierbar herausgestellt und ist die ideale Darstellungsform für den RV, da sie die kardiovaskuläre Morphologie und Physiologie ohne die bekannten Limitationen anderer Bildgebungsverfahren darstellen kann. Diese sind z.B. akustische Fenster, das Körpergewicht oder Narbengewebe bei der Echokardiographie, die Exposition mit ionisierender Strahlung bei der Computertomographie (CT) oder die Invasivität kathetergestützter Diagnostiken (Geva 2011). Die cMRT gilt derzeit als Referenzstandard für die Volumenmessung der rechten Herzkammern bei Patienten mit angeborenen Herzfehlern (Geva 2014).

Zum Vergleich der Inter- und Intraobserver-Variabilitäten der mittels cMRT gemessenen Volumina und der EF bei Gesunden und Patienten mit angeborenen Herzfehlern werden in der folgenden Tabelle (Tabelle 15) die Ergebnisse des CoV für Intraobservertests verschiedener Studien und Patientenkollektive zusammengefasst dargestellt. Der R/L-VI wurde hier aus den Einzelkammervolumina bestimmt. 
Tabelle 15: Vergleich der Intraobserver-Variabilität der biventrikulären Volumina aus MRTUntersuchungen verschiedener Studien (CoV).

\begin{tabular}{|c|c|c|c|c|c|}
\hline Studienpopulation & $\begin{array}{l}\text { Totaler } \\
\text { R/L-VI } \\
\text { CoV(\%) }\end{array}$ & $\operatorname{CoV}(\%)$ & $\operatorname{CoV}(\%)$ & $\begin{array}{l}\text { RV EDV } \\
\operatorname{CoV}(\%)\end{array}$ & $\begin{array}{l}\text { RV ESV } \\
\text { CoV(\%) }\end{array}$ \\
\hline $\begin{array}{l}\text { Diese Studie } 2016 \\
n=6 ; E A\end{array}$ & 3,8 & 2,6 & 2,4 & 3,7 & 9,8 \\
\hline $\begin{array}{l}\text { Rydman et al. } 2017 \\
n=12 ; E A\end{array}$ & & & & 1,4 & 2,4 \\
\hline $\begin{array}{l}\text { Winter et al. } 2008 \\
n=20 ; \text { syst. RV/TGA }\end{array}$ & & & & 6,1 & 6,7 \\
\hline Luijnenburg et al. 2011 & & & & & \\
\hline $\mathrm{n}=35 ;$ angeborene $\mathrm{HF}^{*}$ & & 2,9 & 6,8 & 3,0 & 3,9 \\
\hline $\begin{array}{l}\text { Catalano et al. } 2007 \\
n=45 ; \text { erworbene } \mathrm{HE}\end{array}$ & & & & 7,0 & 9,0 \\
\hline $\begin{array}{l}\text { Clay et al. } 2006 \\
n=20 \text {; erworbene HE }\end{array}$ & & 5,2 & 6,5 & & \\
\hline $\begin{array}{l}\text { Robbers-Visser et al. } 2009 \\
n=15 ; \text { gesunde }\end{array}$ & & 2,1 & 5,8 & 2,1 & 6,0 \\
\hline
\end{tabular}

angeborene $\mathrm{HF}^{*}$ = Vorhofseptumdefekt, Ventrikelseptumdefekt, Transposition der großen Arterien, Double Outlet Right Ventricle, Pulmonalatresie; EA = Ebstein Anomalie; EDV = enddiastolisches Volumen; $\mathrm{ESV}=$ endsystolische Volumen; $\mathrm{HE}=$ Herzerkrankung; $\mathrm{HF}=$ Herzfehler; LV = linker Ventrikel; RV = rechter Ventrikel; syst. = systemisch; TGA = Transposition der großen Arterien. 
In der folgenden Tabelle (Tabelle 16) sind die Ergebnisse des CoV für Interobservertests verschiedener Studien zusammengefasst dargestellt.

Tabelle 16: Vergleich der Interobserver-Variabilität der biventrikulären Volumina aus MRTUntersuchungen verschiedener Studien (CoV).

\begin{tabular}{|c|c|c|c|c|c|}
\hline $\begin{array}{l}\text { Studie } \\
\text { Studienpopulation }\end{array}$ & $\begin{array}{l}\text { Totaler } \\
\text { R/L-VI } \\
\operatorname{CoV}(\%)\end{array}$ & $\begin{array}{l}\text { LV EDV } \\
\operatorname{CoV}(\%)\end{array}$ & $\begin{array}{l}\text { LV ESV } \\
\operatorname{CoV}(\%)\end{array}$ & $\begin{array}{l}\text { RV EDV } \\
\text { CoV(\%) }\end{array}$ & $\begin{array}{l}\text { RV ESV } \\
\text { CoV(\%) }\end{array}$ \\
\hline $\begin{array}{l}\text { Diese Studie } \\
n=6 ; E A\end{array}$ & 2,0 & 5,4 & 6,5 & 8,6 & 14,3 \\
\hline $\begin{array}{l}\text { Rydman et al. } 2017 \\
n=12 ; E A\end{array}$ & & & & 3,0 & 4,5 \\
\hline $\begin{array}{l}\text { Winter et al. } 2008 \\
n=20 ; \text { syst. RV/TGA }\end{array}$ & & & & 10,0 & 12,7 \\
\hline $\begin{array}{l}\text { Luijnenburg et al. } 2011 \\
\mathrm{n}=35 \text {; angeborene } \mathrm{HF}^{*}\end{array}$ & & 4,3 & 10,2 & 4,0 & 7,7 \\
\hline $\begin{array}{l}\text { Gnanappa et al. } 2017 \\
n=120 ; \text { TOF }\end{array}$ & & & & 2,9 & 8,0 \\
\hline $\begin{array}{l}\text { Mooij et al. } 2008 \\
n=60 ; \text { TOF/ASD/gesunde }\end{array}$ & & 3,6 & 10,5 & 6,4 & 13,0 \\
\hline $\begin{array}{l}\text { Catalano et al. } 2007 \\
n=45 ; \text { erworbene HE }\end{array}$ & & & & 8,0 & 11,0 \\
\hline $\begin{array}{l}\text { Clay et al. } 2006 \\
n=20 \text {; erworbene HE }\end{array}$ & & 3,3 & 6,8 & & \\
\hline $\begin{array}{l}\text { Robbers-Visser et al. } 2009 \\
n=15 ; \text { gesunde }\end{array}$ & & 5,3 & 13,9 & 3,4 & 7,6 \\
\hline
\end{tabular}

angeborene $\mathrm{HF}^{*}=$ Vorhofseptumdefekt, Ventrikelseptumdefekt, Transposition der großen Arterien, Double Outlet Right Ventricle, Pulmonalatresie; ASD = Atrium Septum Defekt; EA = Ebstein Anomalie; EDV = enddiastolisches Volumen; ESV = endsystolische Volumen; HE = Herzerkrankung; $\mathrm{HF}=$ Herzfehler; $\mathrm{LV}=$ linker Ventrikel; RV = rechter Ventrikel; syst. = systemisch; TGA = Transposition der großen Arterien; TOF = Fallot'sche Tetralogie.

Der obige Studienvergleich verdeutlicht die unterschiedliche Reproduzierbarkeit von Volumenmessungen der einzelnen Herzhöhlen mittels cMRT. Die Reproduzierbarkeit 
der LV-Volumina fallen insgesamt besser aus als die RV-Volumina (Grothues et al. 2004, Catalano et al. 2007).

Auch in der hier vorgelegten Studie weist die Segmentierung der rechten Herzkammern eine höhere Varianz auf. Bei Patienten mit EA ist die Varianz sogar noch größer als bei Patienten mit anderen angeborenen Herzfehlern oder erworbenen Herzerkrankungen. Dies ist auf das schwierige Einzeichnen des genauen Klappenverlaufs der malformierten TK bei Patienten mit EA zurückzuführen. Die Trennung des RA vom RV gelingt in den bewegten SSFP CINE-Aufnahmen bei Patienten mit EA oft nicht präzise genug. Da das rechte Herz insbesondere bei Patienten mit EA eine komplexe Form hat und der RV stark trabekulisiert ist, hatte unsere Arbeitsgruppe hohe Inter- und Intraobserver-Variabilitäten für den aRV, fRV und das RA erwartet.

Demgegenüber entfällt bei der Ermittlung des R/L-VI die Trennung von Vorhöfen und Ventrikeln durch das genaue Einzeichnen des Verlaufs der TK, was die Volumetrie stark vereinfacht. Die Reproduzierbarkeit des R/L-VI liefert aus diesem Grund deutlich bessere Ergebnisse als die Einzelkammervolumetrie. Der R/L-VI ist somit sogar besser zur Verlaufskontrolle der EA geeignet als die reine Volumetrie.

Zusätzlich liegen ähnlich wie bei den Resultaten der vorliegenden Studie die größten Intra- und Interobserver-Variabilitäten auch bei anderen Autoren bei den biventrikulären ESV (Luijnenburg et al. 2010; Clay et al. 2006; Robbers-Visser 2009; Fratz et al. 2013b). Eine mögliche Erklärung hierfür ist der kleinere absolute Wert des ESV. Absolute Messfehler führen hierdurch zu höheren Observer-Variabilitäten in den ESV im Gegensatz zu den EDV (Luijnenburg et al. 2010). Eine weitere Fehlerquelle ist die endokardiale Grenzziehung, die in der Endsystole schwieriger einzuzeichnen ist, da die Trabekularisierung in dieser Phase dichter ist und die Papillarmuskeln stark kontrahiert sind (Winter et al. 2008).

Fratz et al. (2013b) und Yalonetsky et al. (2011) beschreiben außerdem die Segmentierung von MRT-Bildern von Patienten mit EA in der axialen Ebene als deutlich besser reproduzierbar als die Auswertung anhand von MRT-Bildern in der Kurzachsen-Ebene. Diese Ergebnisse decken sich mit den Resultaten der vorliegenden Studie. Dies ist bei Patienten mit EA darauf zurückzuführen, dass in der axialen Ebene eine bessere Dar- 
stellung der TK und ihrer genauen Insertionspunkte möglich ist (Yalonetsky et al. 2011).

Diesen Ergebnissen zufolge ist es zur Bestimmung des Totalen R/L-VI sinnvoll die enddiastolischen Volumina (EDVi) der jeweiligen Herzkammern zu ermitteln. Bei der Bildgewinnung zeigt sich die axiale Ebene als vorteilhaft, da diese die beste Darstellung der TK ermöglicht.

Auch im Vergleich zu klassischen MRT-Parametern zur Einschätzung der Funktion des rechten Herzens wie der RV-EF zeigt der R/L-VI Vorteile. Die Messung von RV-EF und LV-EF war in dieser Studie wie auch bei anderen Arbeitsgruppen (Grothues et al. 2004; Gnanappa et al. 2017; Rydman et al. 2017) weniger gut reproduzierbar als die Messung der Herzkammervolumina.

Ein Grund hierfür könnte sein, dass eine große Varianz der ermittelten Volumina des rechten Herzens mehrfach in die Berechnung der RV-EF eingeht. Die Aussagekraft der EF als übliches Maß für die Schwere der Herzinsuffizienz wird bei Patienten mit TI zusätzlich relativiert, da es bei jedem Herzschlag zu Blutrückflüssen kommt, welche die Messung der EF beeinflussen. Gerade bei diesen Patienten ist die EF kein geeignetes Maß für die Schwere der Herzinsuffizienz.

Im Gegensatz zur RV-EF erzielt der R/L-VI hervorragende Werte in der Reproduzierbarkeit. Der R/L-VI ist somit besser reproduzierbar als die RV-EF und als CMRT-Parameter zur Einschätzung der Herzfunktion in Verlaufskontrollen zu bevorzugen.

Zusammenfassend ist der Totale R/L-VI ist ein akkurater und reliabler Index und seine sehr gute Reproduzierbarkeit im Gegensatz zur Einzelkammervolumetrie bei Patienten mit EA eine hervorzuhebende Stärke. Der R/L-VI ist bei Patienten mit EA außerdem besser reproduzierbar als die RV-EF und sollte regelmäßig in klinischen Verlaufskontrollen erhoben und zum Erkennen einer fortschreitenden Einschränkung der Herzfunktion der RV-EF gegenüber bevorzugt eingesetzt werden. 


\subsection{Regressionsanalyse zur Beurteilung des Totalen}

\section{Rechts/Links-Volumen-Index als Vorhersageparameter für Ände- rungen in den klinischen Parametern}

Das multivariable Regressionsmodell beschreibt nicht nur den Zusammenhang der untersuchten Parameter mit dem R/L-VI, sondern erlaubt ebenso individuelle Prognosen und die Beurteilung des Gesundheitszustands eines bestimmten Patienten. Durch ein lineares Regressionsmodell können Erwartungswerte für die klinischen Parameter errechnet werden, wobei eine Änderung des Index und der anderen klinischen Parameter berücksichtigt wird (Schneider et al. 2010).

Laut unserer Analyse hatten sich in den letzten drei Jahren weder im R/L-VI noch in den klinischen Untersuchungen größere messbare Änderungen nachweisen lassen, weswegen die Analyse nur eingeschränkt aussagekräftige Ergebnisse liefert.

Der R/L-VI war im genannten Beobachtungszeitraum weder für die Änderung des BNPWertes noch für die Änderung der spiroergometrischen Parameter ein signifikanter Vorhersageparameter. Die Änderung des R/L-VI bezogen auf die Änderung des QRSWertes ergab einen signifikanten $p$-Wert. Demnach würde bei Zunahme des R/L-VI um eine Einheit die QRS Breite leicht abnehmen. Allerdings ist anzumerken, dass laut dem errechneten Bestimmtheitsmaß das Modell nicht ausreichend angepasst ist. Die Aussagen sind demnach nicht verwertbar für Prognosen über den klinischen Verlauf der Erkrankung.

Die Regressionsanalyse ist insgesamt aufgrund der geringen Patientenzahl und der bisher ausgebliebenen klinischen Veränderungen bei den Patienten nur eingeschränkt aussagekräftig.

\subsection{Limitationen der Studie}

Die Anzahl der Patienten und durchgeführten Untersuchungen in dieser Studie waren relativ gering, der Stichprobenumfang dementsprechend klein. Aufgrund der sehr niedrigen Prävalenz der EA war es nicht möglich eine größere Anzahl an Patienten im Raum Niedersachsen zu rekrutieren. Allerdings ist das in dieser Studie inkludierte Patientenkollektiv vergleichbar mit den Zahlen an Patienten anderer aktueller Studien zur 
EA (Attenhofer Jost et al. 2012; Tobler et al. 2013; Fratz et al. 2013b, Lai et al. 2008; Lee et al. 2012).

Aufgrund der sehr niedrigen Prävalenz der Herzerkrankung ist die statistische Aussagekraft generell eingeschränkt. Ein größeres Patientenkollektiv könnte weitere Aufschlüsse sowohl über die Zuverlässigkeit der Schweregradeinteilung anhand des Index, als auch über die Bedeutung des R/L-VI als Prädiktor für das Auftreten schwerer kardialer Komplikationen liefern.

Zudem leiden nur wenige der Patienten in dem hier untersuchten Kollektiv an einer schweren Ausprägungsform der EA mit deutlichen Herzinsuffizienzsymptomen (Hösch 2015). Dies verringert den Grad der Korrelation, und die Aussagekraft des Index für Patienten mit starker Herzinsuffizienzsymptomatik bleibt somit fraglich.

Der Beobachtungszeitraum der Folgestudie ist mit drei Jahren bisher relativ kurz. Weitere Veränderungen im körperlichen Zustand der Patienten sind abzuwarten und eine mögliche Zunahme der Kammervolumina ist weiterhin zu verfolgen.

Die Untersuchungen wurden nur an erkrankten Patienten durchgeführt, es fehlte eine gesunde Vergleichsgruppe. Die Referenzwerte des R/L-VI für Gesunde wurden allerdings für den ersten Teil der Studie anhand einer Literaturrecherche generiert.

Auf eine ausführlichere statistische Analyse der Einflussfaktoren in der RegressionsanaIyse wurde aufgrund der geringen Veränderungen verzichtet. Zusätzliche Informationen könnte ein weiteres multiples Regressionsmodell bei den folgenden Follow-UpStudien liefern und die Bedeutung des R/L-VI als Vorhersageparameter für den klinischen Verlauf der Erkrankung verifiziert werden.

Zukünftig wäre eine prospektive Längsschnitt-Multicenter-Studie ideal, um eine stärkere statistische Aussagekraft zu erreichen (Hösch 2015). Dadurch könnte auch der Zusammenhang des R/L-VI mit dem klinischen Verlauf der Erkrankung und die Bedeutung des Index für die Festlegung eines optimalen Therapiezeitpunktes besser evaluiert werden. 


\section{Zusammenfassung und Ausblick}

Durch die vorliegende prospektive Studie wird der neu eingeführte Totale Rechts/Links-Volumen-Index validiert, welcher als Bildgebungs-Äquivalent zur Schweregradeinteilung der Ebstein Anomalie dienen soll.

Der Rechts/Links-Volumen-Index zeigt über die ersten drei Jahre der Follow-Up-Studie konsistente Werte von im Mittel 2,6 $\pm 1,3$. Der neue Index ist über die ersten drei Jahre des Beobachtungszeitraums stabil und nach ersten Einschätzungen ein geeignetes bildgebungsbasiertes Äquivalent für die Schweregradeinteilung der Ebstein Anomalie. Der Rechts/Links-Volumen-Index zeigt eine gute Korrelation mit klinischen Parametern wie der QRS-Zeit als Zeichen für eine verzögerte Erregungsausbreitung in einem vergrößerten rechten Ventrikel sowie mit spiroergometrischen Belastungsparametern wie dem exspiratorischen $\mathrm{CO}_{2}$-Quotienten und der maximalen Sauerstoffaufnahme, die bei Erwachsenen mit angeborenen Herzfehlern als Prädiktoren für einen ungünstigen Krankheitsverlauf angesehen werden (Diller 2005; Hösch 2015). Der Index kann damit bei Patienten mit Ebstein Anomalie als Frühmarker zum Aufzeigen einer Verschlechterung der körperlichen Leistungsfähigkeit bereits vor einer fortgeschrittenen Herzinsuffizienz angesehen werden.

In den Einzelkammermessungen mittels kardialer Magnetresonanztherapie vergrößerte sich das Volumen des funktionellen rechten Ventrikels im Kollektiv signifikant, während sich dessen Ejektionsfraktion verringerte. Dies spricht für eine fortschreitende Vergrößerung des rechten Herzens und eine Abnahme der rechtsventrikulären Funktion im Verlauf der Erkrankung. Durch die regelmäßige Ermittlung des Rechts/LinksVolumen-Index kann eine Änderung in der Herzgröße möglicherweise frühzeitig festgestellt und in Behandlungsentscheidungen miteinbezogen werden.

Das Kaplan-Meier-Verfahren zeigte ein erhöhtes Risiko für des Eintreten kardialer Ereignisse bei Patienten mit einem höheren Schweregrad der Ebstein Anomalie (Rechts/Links-Volumen-Index von > 2,0). Dies spricht für eine gute Anwendbarkeit des Index als Prädiktor für bevorstehende kardiale Ereignisse und indiziert eine engmaschigere klinische Kontrolle insbesondere hinsichtlich Herzrhythmusstörungen und Leistungsfähigkeit von Patienten mit einem Rechts/Links-Volumen-Index von > 2,0. 
Die Reproduzierbarkeit der Magnetresonanztherapie-Datenauswertung für die Einzelkammervolumina war ausreichend akkurat und trotz höheren Schwankungen in den Volumina der rechten Herzkammern erzielte der Rechts/Links-Volumen-Index exzellente Werte in der Reproduzierbarkeit. Eine vereinfachte Ermittlung des Index ohne aufwendige Einzelkammersegmentierung durch das Umfahren der Gesamtkonturen der rechten und linken Herzhälften ist möglich und diese simple Methode ebenfalls sehr gut reproduzierbar.

Zusätzlich zeigt sich der Rechts/Links-Volumen-Index bei Patienten mit Ebstein Anomalie besser reproduzierbar als die rechtsventrikuläre Ejektionsfraktion. Dies lässt annehmen, dass der neue Parameter bei Patienten mit Ebstein Anomalie eine ventrikuläre Dysfunktion in der Verlaufsuntersuchung besser anzeigen kann als klassische kardiale Magnetresonanztherapie-Funktionsparameter wie die rechtsventrikuläre Ejektionsfraktion.

Da im Beobachtungszeitraum weder in den klinischen Parametern noch im Rechts/Links-Volumen-Index signifikante Veränderungen eintraten zeigten sich im multiplen Regressionsmodell keine statistisch signifikante Assoziation zwischen Index und klinischen Parametern.

Insgesamt ist der Rechts/Links-Volumen-Index ein einfach zu bestimmender Parameter in der Überwachung des Krankheitsverlaufs bei Patienten mit Ebstein'scher Anomalie. Er könnte zukünftig dazu beitragen, den optimalen Zeitpunkt einer therapeutischen Intervention bei Patienten mit Ebstein Anomalie zu bestimmen.

Neben den Ergebnissen der körperlichen und spiroergometrischen Untersuchungen, der New York Heart Association-Klassifikation und den echokardiographischen Parametern ist die Bestimmung des Rechts/Links-Volumen-Index ein gutes diagnostisches Mittel zur Überwachung des Krankheitsverlaufs und ein Wegweiser für das klinische Management von Patienten mit Ebstein Anomalie. Der Rechts/Links-Volumen-Index ist ein hoffnungstragender Baustein auf dem Weg zu klareren Definitionen für konkrete Indikationen und den passenden Zeitpunkt einer angemessenen Therapie. Darum wird vorgeschlagen, den neuen Rechts/Links-Volumen-Index neben den etablierten kardialen Markern der Herzinsuffizienz in die klinische Routineuntersuchung von Patienten mit Ebstein'scher Anomalie aufzunehmen. 


\section{Literaturverzeichnis}

Ahmed S, Nanda NC, Nekkanti R, Pacifico AD (2003): Transesophageal threedimensional echocardiographic demonstration of Ebstein's anomaly. Echocardiogr Mt Kisco N 20, 305-307

Anderson KR, Zuberbuhler JR, Anderson RH, Becker AE, Lie JT (1979): Morphologic spectrum of Ebstein's anomaly of the heart: a review. Mayo Clin Proc 54, 174180

Alfakih K, Plein S, Bloomer T, Jones T, Ridgway J, Sivananthan M (2003): Comparison of right ventricular volume measurements between axial and short axis orientation using steady-state free precession magnetic resonance imaging. J Magn Reson Imaging 18, 25-32

Assenza GE, Valente AM, Geva T, Graham D, Romana Pluchinotta F, Sanders SP, Autore C, Volpe M, Landzberg MJ, Cecchin F (2013): QRS duration and QRS fractionation on surface electrocardiogram are markers of right ventricular dysfunction and atrialization in patients with Ebstein anomaly. Eur Heart J 34, 191-200

Attenhofer Jost CH, Connolly HM, O'Leary PW, Warnes CA, Tajik AJ, Seward JB (2005): Left heart lesions in patients with Ebstein anomaly. Mayo Clin Proc 80, 361-368

Attenhofer Jost CH, Connolly HM, Dearani JA, Edwards WD, Danielson GK (2007): Ebstein's anomaly. Circulation 115, 277-285

Attenhofer Jost CH, Edmister WD, Julsrud PR, Dearani JA, Savas Tepe M, Warnes CA, Scott CG, Anavekar NS, Ammash NM, Connolly HM (2012): Prospective comparison of echocardiography versus cardiac magnetic resonance imaging in patients with Ebstein's anomaly. Int J Cardiovasc Imaging 28, 1147-1159

Attenhofer Jost CH, Tan NY, Hassan A, Vargas ER, Hodge DO, Dearani JA, Connolly H, Asirvatham SJ, McLeod CJ (2018): Sudden death in patients with Ebstein anomaly. Eur Heart J 39, 1970-1977a

Attie F, Rosas M, Rijlaarsdam M, Buendia A, Zabal C, Kuri J, Granados N (2000): The adult patient with Ebstein anomaly. Outcome in 72 unoperated patients. Medicine (Baltimore) 79, 27-36

Benson DW, Silberbach GM, Kavanaugh-McHugh A, Cottrill C, Zhang Y, Riggs S, Smalls O, Johnson MC, Watson MS, Seidman JG et al. (1999): Mutations in the cardiac transcription factor NKX2.5 affect diverse cardiac developmental pathways. J Clin Invest 104, 1567-1573

Bharucha T, Anderson RH, Lim ZS, Vettukattil JJ (2010): Multiplanar review of threedimensional echocardiography gives new insights into the morphology of Ebstein's malformation. Cardiol Young 20, 49-53 
Bland JM, Altman D (1986): Statistical methods for assessing agreement between two methods of clinical measurement. The Lancet 327, 307-310

Brickner ME, Hillis LD, Lange RA (2000): Congenital heart disease in adults. Second of two parts. N Engl J Med 342, 334-342

Brown ML, Dearani JA, Danielson GK, Cetta F, Connolly HM, Warnes CA, Li Z, Hodge DO, Driscoll DJ, Mayo Clinic Congenital Heart Center (2008): The outcomes of operations for 539 patients with Ebstein anomaly. J Thorac Cardiovasc Surg 135, 1120-1137

Brown ML, Dearani JA, Burkhart HM (2009): The adult with congenital heart disease: medical and surgical considerations for management. Curr Opin Pediatr 21, 561-564

Bluemke DA, Boxerman JL, Atalar E, McVeigh ER (1997): Segmented K-space cine breath-hold cardiovascular MR imaging: Part 1. Principles and technique. AJR Am J Roentgenol 169, 395-400

Bredy C, Ministeri M, Kempny A, Alonso-Gonzalez R, Swan L, Uebing A, Diller GP, Gatzoulis MA, Dimopoulos K (2017): NYHA classification in adults with congenital heart disease: Relation to objective measures of exercise and outcome. Eur Heart J Qual Care Clin Outcomes 4, 51-58

Cappato R, Schlüter M, Weiss C, Antz M, Koschyk DH, Hofmann T, Kuck KH (1996): Radiofrequency current catheter ablation of accessory atrioventricular pathways in Ebstein's anomaly. Circulation 94, 376-383

Carpentier A, Chauvaud S, Macé L, Relland J, Mihaileanu S, Marino JP, Abry B, Guibourt $P$ (1988): A new reconstructive operation for Ebstein's anomaly of the tricuspid valve. J Thorac Cardiovasc Surg 96, 92-101

Catalano O, Antonaci S, Opasich C, Moro G, Mussida M, Perotti M, Calsamiglia G, Frascaroli M, Baldi M, Cobelli $F$ (2007): Intra-observer and interobserver reproducibility of right ventricle volumes, function and mass by cardiac magnetic resonance. J Cardiovasc Med 8, 807-814

Celermajer DS, Cullen S, Sullivan ID, Spiegelhalter DJ, Wyse RK, Deanfield JE (1992):

Outcome in neonates with Ebstein's anomaly. J Am Coll Cardiol 19, 1041-1046

Celermajer DS, Bull C, Till JA, Cullen S, Vassillikos VP, Sullivan ID, Allan L, Nihoyannopoulos P, Somerville J, Deanfield JE (1994): Ebstein's anomaly: presentation and outcome from fetus to adult. J Am Coll Cardiol 23, 170-176

Cerqueira MD, Weissman NJ, Dilsizian V, Jacobs AK, Kaul S, Laskey WK, Pennell DJ, Rumberger JA, Ryan T, Verani MS (2002): Standardized myocardial segmentation and nomenclature for tomographic imaging of the heart. A statement for healthcare professionals from the Cardiac Imaging Committee of the Council on Clinical Cardiology of the American Heart Association. Circulation 105, 539-542 
Chauvaud S, Berrebi A, d'Attellis N, Mousseaux E, Hernigou A, Carpentier A (2003): Ebstein's anomaly: repair based on functional analysis. Eur J Cardio-Thorac Surg Off J Eur Assoc Cardio-Thorac Surg 23, 525-531

Clay S, Alfakih K, Messroghli DR, Jones T, Ridgway JP, Sivananthan MU (2006): The reproducibility of left ventricular volume and mass measurements: a comparison between dual-inversion-recovery black-blood sequence and SSFP. Eur Radiol 16, 32-37

Cohen LS, Friedman JM, Jefferson JW, Johnson EM, Weiner ML (1994): A reevaluation of risk of in utero exposure to lithium. JAMA 271, 146-150

Correa-Villaseñor A, Ferencz C, Neill CA, Wilson PD, Boughman JA (1994): Ebstein's malformation of the tricuspid valve: genetic and environmental factors. The Baltimore-Washington Infant Study Group. Teratology 50, 137-147

Danielson GK, Driscoll DJ, Mair DD, Warnes CA, Oliver WC (1992): Operative treatment of Ebstein's anomaly. J Thorac Cardiovasc Surg 104, 1195-1202

da Silva JP, Baumgratz JF, da Fonseca L, Franchi SM, Lopes LM, Tavares GMP, Soares AM, Moreira LF, Barbero-Marcial M (2007): The cone reconstruction of the tricuspid valve in Ebstein's anomaly. The operation: early and midterm results. J Thorac Cardiovasc Surg 133, 215-223

da Silva JP, da Silva L da F (2012): Ebstein's anomaly of the tricuspid valve: the cone repair. Semin Thorac Cardiovasc Surg Pediatr Card Surg Annu 15, 38-45

Delhaas T, Sarvaas GJ du M, Rijlaarsdam ME, Strengers JL, Eveleigh RM, Poulino SE, Korte CL de, Kapusta L (2010): A Multicenter, Long-Term Study on Arrhythmias in Children with Ebstein Anomaly. Pediatr Cardiol 31, 229-233

Dewey M, Müller M, Eddicks S, Schnapauff D, Teige F, Rutsch W, Borges AC, Hamm B (2006): Evaluation of global and regional left ventricular function with 16-slice computed tomography, biplane cineventriculography, and two-dimensional transthoracic echocardiography: comparison with magnetic resonance imaging. J Am Coll Cardiol 48, 2034-2044

DGPK (2014): Ebstein-Anomalie der Trikuspidalklappe bei Kindern und Jugendlichen (Leitlinie Pädiatrische Kardiologie). S2k-Leitlinie der Deutschen Gesellschaft für Pädiatrische Kardiologie. https://www.awmf.org/uploads/tx_szleitlinien/023041I_S2k_Ebstein_Anomalie_Kinder_Jugendliche_2016-05-abgelaufen.pdf; abgerufen am 05.11.2017

Diller G, Dimopoulos K, Okonko D, Li W, Babu-Narayan SV, Broberg CS, Johansson B, Bouzas B, Mullen MJ, Poole-Wilson PA et al. (2005): Exercise intolerance in adult congenital heart disease: comparative severity, correlates, and prognostic implication. Circulation 112, 828-835 
Diller G-P, Dimopoulos K, Okonko D, Uebing A, Broberg CS, Babu-Narayan S, Bayne S, Poole-Wilson PA, Sutton R, Francis DP, Gatzoulis MA (2006): Heart Rate Response During Exercise Predicts Survival in Adults With Congenital Heart Disease. J Am Coll Cardiol 48, 1250-1256

Dimopoulos K, Okonko DO, Diller G-P, Broberg CS, Salukhe TV, Babu-Narayan SV, Li W, Uebing A, Bayne S, Wensel R et al. (2006): Abnormal ventilatory response to exercise in adults with congenital heart disease relates to cyanosis and predicts survival. Circulation 113, 2796-2802

Ebstein W (1866): Ueber einen sehr seltenen Fall von Insufficienz der Valvula tricuspidalis, bedingt durch eine angeborene hochgradige Mißbildung derselben. Arch Anat Physiol, 238-254

Edwards WD (1993): Embryology and Pathologic Features of Ebstein's Anomaly. Prog Pediatr Cardiol 2, 5-15

Engelen K, Postma AV, Meerakker JBA, Roos-Hesselink JW, Helderman-van den Enden ATJM, Vliegen HW, Rahman T, Baars MJH, Sels J, Bauer U et al. (2013): Ebstein's anomaly may be caused by mutations in the sarcomere protein gene MYH7. Neth Heart J 21, 113-117

Engle MA, Payne T, Bruins C, Taussig HB (1950): Ebstein's anomaly of the tricuspid valve; report of three cases and analysis of clinical syndrome. Circulation $\underline{1}$, $1246-1260$

Fratz S, Chung T, Greil GF, Samyn MM, Taylor AM, Valsangiacomo Buechel ER, Yoo S, Powell AJ (2013a): Guidelines and protocols for cardiovascular magnetic resonance in children and adults with congenital heart disease: SCMR expert consensus group on congenital heart disease. J Cardiovasc Magn Reson 15, 51-51

Fratz S, Janello C, Müller D, Seligmann M, Meierhofer C, Schuster T, Schreiber C, Martinoff S, Hess J, Kühn A et al. (2013b): The functional right ventricle and tricuspid regurgitation in Ebstein's anomaly. Int J Cardiol 167, 258-261

Gentles TL, Calder AL, Clarkson PM, Neutze JM (1992): Predictors of long-term survival with Ebstein's anomaly of the tricuspid valve. Am J Cardiol 69, 377-381

Geva T (2011): Repaired tetralogy of Fallot: the roles of cardiovascular magnetic resonance in evaluating pathophysiology and for pulmonary valve replacement decision support. J Cardiovasc Magn Reson Off J Soc Cardiovasc Magn Reson 13, 9-9

Geva T (2014): Is MRI the Preferred Method for Evaluating Right Ventricular Size and Function in Patients With Congenital Heart Disease?: MRI Is the Preferred Method for Evaluating Right Ventricular Size and Function in Patients With Congenital Heart Disease. Circ Cardiovasc Imaging 7, 190-197 
Giuliani ER, Fuster V, Brandenburg RO, Mair DD (1979): Ebstein's anomaly: the clinical features and natural history of Ebstein's anomaly of the tricuspid valve. Mayo Clin Proc 54, 163-173

Gnanappa GK, Rashid I, Celermajer D, Ayer J, Puranik R (2017): Reproducibility of Cardiac Magnetic Resonance Imaging (CMRI)-Derived Right Ventricular Parameters in Repaired Tetralogy of Fallot (ToF). Heart Lung Circ 27, 381-385

Goldman MR, Pohost GM, Ingwall JS, Fossel ET (1980): Nuclear magnetic resonance imaging: potential cardiac applications. Am J Cardiol 46, 1278-1283

Goleski PJ, Sheehan FH, Chen SSM, Kilner PJ, Gatzoulis MA (2014): The shape and function of the left ventricle in Ebstein's anomaly. Int J Cardiol 171, 404-412

Grothues F, Moon JC, Bellenger NG, Smith GS, Klein HU, Pennell DJ (2004): Interstudy reproducibility of right ventricular volumes, function, and mass with cardiovascular magnetic resonance. Am Heart J 147, 218-223

Gutberlet M, Oellinger $H$, Ewert $P$, Nagdyman N, Amthauer $H$, Hoffmann T, Hetzer R, Lange $P$, Felix R (2000): Prä- und postoperative Beurteilung der ventrikulären Funktion, Muskelmasse und Klappenmorphologie bei der Ebstein-Anomalie mit der Magnetresonanztomographie. Rofo 172, 436-442

Hawkes RC, Holland GN, Moore WS, Roebuck EJ, Worthington BS (1981): Nuclear magnetic resonance (NMR) tomography of the normal heart. J Comput Assist Tomogr $\underline{5}, 605-612$

Hebe J (2000): Ebstein's anomaly in adults. Arrhythmias: diagnosis and therapeutic approach. Thorac Cardiovasc Surg 48, 214-219

Ho SY, Goltz D, McCarthy K, Cook AC, Connell MG, Smith A, Anderson RH (2000): The atrioventricular junctions in Ebstein malformation. Heart Br Card Soc 83, 444449

Hösch O, Sohns JM, Nguyen T, Lauerer P, Rosenberg C, Kowallick JT, Kutty S, Unterberg C, Schuster A, Faßhauer M et al. (2014): The Total Right/Left-Volume-Index: A New and Simplified Cardiac Magnetic Resonance Measure to Evaluate the Severity of Ebstein's Anomaly of the Tricuspid Valve: A Comparison with Heart Failure Markers from Various Modalities. Circ Cardiovasc Imaging 7, 601-609

Hösch O: Der Totale Rechts/Links-Volumen-Index: ein neuer MRT-Parameter zur Evaluation des Schweregrads der Ebstein-Anomalie - ein prospektiver Vergleich mit verschiedenen Herzinsuffizienzparametern. Med. Diss. Göttingen 2015

Hösch O, Ngyuen T-T, Lauerer P, Schuster A, Kutty S, Staab W, Unterberg-Buchwald C, Sohns JM, Paul T, Lotz J, Steinmetz M (2015b): BNP and haematological parameters are markers of severity of Ebstein's anomaly: correlation with CMR and cardiopulmonary exercise testing. Eur Heart J Cardiovasc Imaging 16, 670-675 
Hudsmith LE, Petersen SE, Francis JM, Robson MD, Neubauer S (2005): Normal human left and right ventricular and left atrial dimensions using steady state free precession magnetic resonance imaging. J Cardiovasc Magn Reson Off J Soc Cardiovasc Magn Reson 7, 775-782

Hünerbein R: Radiologische Verfahren - Magnetresonanztomografie. In: Reiser M, Kuhn F, Debus J (Hrsg.): Radiologie (Duale Reihe). 3., vollst. überarb. u. erw. Auflage; Thieme, Stuttgart 2011, 79-83

Kaplan EL, Meier P (1985): Nonparametric estimation from incomplete observations. JASA 53, 457-481.

Kawase I, Ozaki S, Kawada M (2008): Repair of Ebstein's anomaly in an elderly patient; report of a case. Kyobu Geka 61, 887-890

Kellman P, Chefd'hotel C, Lorenz CH, Mancini C, Arai AE, McVeigh ER (2009): High spatial and temporal resolution cardiac cine MRI from retrospective reconstruction of data acquired in real time using motion correction and resorting. Magn Reson Med 62, 1557-1564

Khairy P, Van Hare GF, Balaji S, Berul Cl, Cecchin F, Cohen MI, Daniels CJ, Deal BJ, Dearani JA, Groot N de et al. (2014): PACES/HRS expert consensus statement on the recognition and management of arrhythmias in adult congenital heart disease: developed in partnership between the Pediatric and Congenital Electrophysiology Society (PACES) and the Heart Rhythm Society (HRS). Endorsed by the governing bodies of PACES, HRS, the American College of Cardiology (ACC), the American Heart Association (AHA), the European Heart Rhythm Association (EHRA), the Canadian Heart Rhythm Society (CHRS), and the International Society for Adult Congenital Heart Disease (ISACHD). Can J Cardiol 30, e1-e63

Kilner PJ, Geva T, Kaemmerer H, Trindade PT, Schwitter J, Webb GD (2010): Recommendations for cardiovascular magnetic resonance in adults with congenital heart disease from the respective working groups of the European Society of Cardiology. Eur Heart J 31, 794-805

Kiziltan HT, Theodoro DA, Warnes CA, O'Leary PW, Anderson BJ, Danielson GK (1998): Late results of bioprosthetic tricuspid valve replacement in Ebstein's anomaly. Ann Thorac Surg 66, 1539-1545

Knott-Craig CJ, Overholt ED, Ward KE, Ringewald JM, Baker SS, Razook JD (2002): Repair of Ebstein's anomaly in the symptomatic neonate: an evolution of technique with 7-year follow-up. Ann Thorac Surg 73, 1786-1793

Kohn W: Statistik: Datenanalyse und Wahrscheinlichkeitsrechnung. Springer, Berlin Heidelberg 2005 
Koike A, Hiroe M, Adachi H, Yajima T, Nogami A, Ito H, Takamoto T, Taniguchi K, Marumo $F$ (1992): Anaerobic metabolism as an indicator of aerobic function during exercise in cardiac patients. J Am Coll Cardiol 20, 120-126

Kramer CM, Barkhausen J, Flamm SD, Kim RJ, Nagel E, Society for Cardiovascular Magnetic Resonance Board of Trustees Task Force on Standardized Protocols (2013): Standardized cardiovascular magnetic resonance (CMR) protocols 2013 update. J Cardiovasc Magn Reson Off J Soc Cardiovasc Magn Reson 15, 91-91

Lackner K, Steudel A, Krahe T, Becher H, Gieseke J, Thurn P (1987): Kardio-MRT. Untersuchungstechnik und topographisch-anatomischer Bildinhalt. Rofo $\underline{146}$, 249255

Lai WW, Powell AJ, Geva T (2008): 1058 The extent of tricuspid valve septal displacement adversely affects right ventricular function in Ebstein anomaly: a cardiac MRI study. J Cardiovasc Magn Reson 10, A183

Lamers WH, Virágh S, Wessels A, Moorman AFM, Anderson RH (1995): Formation of the Tricuspid Valve in the Human Heart. Circulation 91, 111-121

Lange R, Burri M, Eschenbach LK, Badiu CC, da Silva JP, Nagdyman N, Fratz S, Hörer J, Kühn A, Schreiber C, Vogt MO (2015): Da Silva's cone repair for Ebstein's anomaly: effect on right ventricular size and function. Eur J Cardio-Thorac Surg Off J Eur Assoc Cardio-Thorac Surg 48, 316-321

Lee CM, Sheehan FH, Bouzas B, Chen SSM, Gatzoulis MA, Kilner PJ (2012): The shape and function of the right ventricle in Ebstein's anomaly. Int J Cardiol 167, 704710

Lindinger A, Schwedler G, Hense H (2010): Prevalence of congenital heart defects in newborns in Germany: Results of the first registration year of the PAN Study (July 2006 to June 2007). Klin Padiatr 222, 321-326

Luijnenburg SE, Robbers-Visser D, Moelker A, Vliegen HW, Mulder BJM, Helbing WA (2010): Intra-observer and interobserver variability of biventricular function, volumes and mass in patients with congenital heart disease measured by CMR imaging. Int J Cardiovasc Imaging 26, 57-64

MacLellan-Tobert SG, Driscoll DJ, Mottram CD, Mahoney DW, Wollan PC, Danielson GK (1997): Exercise tolerance in patients with Ebstein's anomaly. J Am Coll Cardiol 29, 1615-1622

Makous N, Vander Veer JB (1966): Ebstein's anomaly and life expectancy. Am J Cardiol $18,100-104$

Mann RJ, Lie JT (1979): The life story of Wilhelm Ebstein (1836-1912) and his almost overlooked description of a congenital heart disease. Mayo Clin Proc 54, 197204 
Mooij CF, Wit CJ de, Graham DA, Powell AJ, Geva T (2008): Reproducibility of MRI Measurements of Right Ventricular Size and Function in Patients with Normal and Dilated Ventricles. J Magn Reson Imaging JMRI 28, 67-67

Moon JCC, Lorenz CH, Francis JM, Smith GC, Pennell DJ (2002): Breath-hold FLASH and FISP Cardiovascular MR Imaging: Left Ventricular Volume Differences and Reproducibility. Radiology 223, 789-797

Müller J, Kühn A, Vogt M, Schreiber C, Hess J, Hager A (2011): Improvements in exercise performance after surgery for Ebstein anomaly. J Thorac Cardiovasc Surg 141, 1192-1195

Negoi RI, Ispas AT, Ghiorghiu I, Filipoiu F, Negoi I, Hostiuc M, Hostiuc S, Ginghina C (2013): Complex Ebstein's malformation: defining preoperative cardiac anatomy and function. J Card Surg 28, 70-81

Paranon S, Acar P (2008): Ebstein's anomaly of the tricuspid valve: from fetus to adult: congenital heart disease. Heart 94, 237-243

Postma AV, van Engelen K, van de Meerakker J, Rahman T, Probst S, Baars MJH, Bauer U, Pickardt T, Sperling SR, Berger F et al. (2011): Mutations in the sarcomere gene MYH7 in Ebstein anomaly. Circ Cardiovasc Genet 4, 43-50

Radojevic J, Inuzuka R, Alonso-Gonzalez R, Borgia F, Giannakoulas G, Prapa M, Liodakis E, Li W, Swan L, Diller GP et al. (2013): Peak oxygen uptake correlates with disease severity and predicts outcome in adult patients with Ebstein's anomaly of the tricuspid valve. Int J Cardiol 163, 305-308

Reemtsen BL, Polimenakos AC, Fagan BT, Wells WJ, Starnes VA (2007): Fate of the right ventricle after fenestrated right ventricular exclusion for severe neonatal Ebstein anomaly. J Thorac Cardiovasc Surg 134, 1406-1412

Reich JD, Auld D, Hulse E, Sullivan K, Campbell R (1998): The Pediatric Radiofrequency Ablation Registry's experience with Ebstein's anomaly. Pediatric Electrophysiology Society. J Cardiovasc Electrophysiol 9, 1370-1377

Ressing M, Blettner M, Klug SJ (2010): Data analysis of epidemiological studies: part 11 of a series on evaluation of scientific publications. Dtsch Arzteblatt Int 107, 187-192

Ridgway JP (2010): Cardiovascular magnetic resonance physics for clinicians: part I. J Cardiovasc Magn Reson Off J Soc Cardiovasc Magn Reson 12, 71-71

Robbers-Visser D, Boersma E, Helbing WA (2009): Normal biventricular function, volumes, and mass in children aged 8 to 17 years. J Magn Reson Imaging JMRI 29, 552-559

Rodgers CT, Robson MD (2011): Cardiovascular magnetic resonance: physics and terminology. Prog Cardiovasc Dis 54, 181-190 
Rostagno C, Galanti G, Comeglio M, Boddi V, Olivo G, Serneri GGN (2000): Comparison of different methods of functional evaluation in patients with chronic heart failure. Eur J Heart Fail 2, 273-280

Rydman R, Shiina Y, Diller G-P, Niwa K, Li W, Uemura H, Uebing A, Barbero U, Bouzas B, Ernst $S$ et al. (2017): Major adverse events and atrial tachycardia in Ebstein's anomaly predicted by cardiovascular magnetic resonance. Heart Br Card Soc 104, 37-44

Saeed M, Van TA, Krug R, Hetts SW, Wilson MW (2015): Cardiac MR imaging: current status and future direction. Cardiovasc Diagn Ther $\underline{5}, 290-310$

Sarikouch S, Peters B, Gutberlet M, Leismann, B., Kelter-Kloepping A, Koerperich H, Kuehne T, Beerbaum P (2010): Sex-Specific Pediatric Percentiles for Ventricular Size and Mass as Reference Values for Cardiac MRI: Assessment by SteadyState Free-Precession and Phase-Contrast MRI Flow. Circ Cardiovasc Imaging $\underline{3}$, 65-76

Sarikouch S, Körperich H, Böthig D, Peters B, Lotz J, Gutberlet M, Beerbaum P, Kühne T (2011): Reference values for atrial size and function in children and young adults by cardiac MR: a study of the German competence network congenital heart defects. J Magn Reson Imaging 33, 1028-1039

Schneider A, Hommel G, Blettner M (2010): Linear regression analysis: part 14 of a series on evaluation of scientific publications. Dtsch Arzteblatt Int 107, 776-782

Sievers B, Addo M, Breuckmann F, Barkhausen J, Erbel R (2007): Reference Right Atrial Function Determined by Steady-State Free Precession Cardiovascular Magnetic Resonance. J Cardiovasc Magn Reson 9, 807-814

Shrout PE, Fleiss JL (1979): Intraclass correlations: uses in assessing rater reliability. Psychol Bull 86, 420-428

Sung RJ, Tamer DM, Agha AS, Castellanos A, Myerburg RJ, Gelband H (1975): Etiology of the electrocardiographic pattern of "incomplete right bundle branch block" in atrial septal defect: An electrophysiologic study. J Pediatr 87, 1182-1186

Surkova E, Muraru D, Iliceto S, Badano LP (2016): The use of multimodality cardiovascular imaging to assess right ventricular size and function. Int J Cardiol 214, 5469

Tobler D, Yalonetsky S, Crean AM, Granton JT, Burchill L, Silversides CK, Wald RM (2013): Right heart characteristics and exercise parameters in adults with Ebstein anomaly: New perspectives from cardiac magnetic resonance imaging studies. Int J Cardiol 165, 146-150 
Trojnarska O, Szyszka A, Gwizdała A, Siniawski A, Oko-Sarnowska Z, Chmara E, Straburzyńska-Migaj E, Katarzyński S, Cieśliński A (2006): Adults with Ebstein's anomaly-Cardiopulmonary exercise testing and BNP levels: Exercise capacity and BNP in adults with Ebstein's anomaly. Int J Cardiol 111, 92-97

Vacca JB, Bussmann DW, Mudd JG (1958): Ebstein's anomaly; complete review of 108 cases. Am J Cardiol 2, 210-226

Watson H (1974): Natural history of Ebstein's anomaly of tricuspid valve in childhood and adolescence. An international co-operative study of 505 cases. Br Heart J $36,417-427$

Winter MM, Bernink FJ, Groenink M, Bouma BJ, van Dijk AP, Helbing WA, Tijssen JG, Mulder BJ (2008): Evaluating the systemic right ventricle by CMR: the importance of consistent and reproducible delineation of the cavity. J Cardiovasc Magn Reson 10, 40-40

Wirtz M, Caspar F: Beurteilerübereinstimmung und Beurteilerreliabilität. Hogrefe, München 2002.

WMA (World Medical Association) (2013): Declaration of Helsinki: ethical principles for medical research involving human subjects. JAMA 310, 2191-2194

Van Gestel A, Teschler H: Physiotherapie bei chronischen Atemwegs- und Lungenerkrankungen: Evidenzbasierte Praxis. 2. Auflage; Springer, Berlin 2014

Yalonetsky S, Tobler D, Greutmann M, Crean AM, Wintersperger BJ, Nguyen ET, Oechslin EN, Silversides CK, Wald RM (2011): Cardiac Magnetic Resonance Imaging and the Assessment of Ebstein Anomaly in Adults. Am J Cardiol 107, 767773

Yang H, Lee CLY, Young DC, Shortliffe M, Yu W, Wright JR (2004): A rare case of interstitial del(1)(p34.3p36.11) diagnosed prenatally. Fetal Pediatr Pathol 23, 251-255

Yatsenko SA, Yatsenko AN, Szigeti K, Craigen WJ, Stankiewicz P, Cheung SW, Lupski JR (2004): Interstitial deletion of 10p and atrial septal defect in DiGeorge 2 syndrome. Clin Genet 66, 128-136

Zhou X, Thavendiranathan P, Chen Y, Cheng L, Qian Z, Liu S, Houle H, Zhi G, Vannan MA (2016): Feasibility of Automated Three-Dimensional Rotational Mechanics by Real-Time Volume Transthoracic Echocardiography: Preliminary Accuracy and Reproducibility Data Compared with Cardiovascular Magnetic Resonance. J Am Soc Echocardiogr Off Publ Am Soc Echocardiogr 29, 62-73

Zuberbuhler JR, Becker AE, Anderson RH, Lenox CC (1984): Ebstein's malformation and the embryological development of the tricuspid valve. With a note on the nature of "clefts" in the atrioventricular valves. Pediatr Cardiol 5, 289-2 


\section{Danksagung}

In besonderem Maße danke ich meinem Doktorvater, Herrn PD Dr. med. Michael Steinmetz, der mir die Durchführung der Promotion in der Arbeitsgruppe der Pädiatrischen Kardiologie und Intensivmedizin ermöglicht und mich von Beginn an geduldig begleitet hat. Herr PD Dr. med. Steinmetz hat mich kontinuierlich unterstützt und stand mir stets mit Rat und Tat zur Seite. Mein besonderer Dank gilt ihm für die hervorragende Betreuung, Expertise, Motivation und das hohe Maß an eigenem Zeitaufwand bis zur Fertigstellung der hier vorliegenden Dissertation.

Ich danke auch besonders Frau Dr. med. Olga Hösch für die Erläuterung der Theorie und Methodik der Studie und die zeitaufwendigen und gründlichen Praxisanleitungen der MRT-Datenauswertung, sowie die optimale Vorarbeit und Patientenrekrutierung.

Dem gesamten Team der Kinderkardiologischen Poliklinik sowie dem MRT-Team, insbesondere Frau Tanja Otto, gebührt ebenfalls mein Dank. Durch ihre Arbeit sind exzellente MRT-Bilder entstanden, die für die Auswertung unentbehrlich waren. Danke auch für die kollegialen Hilfestellungen und zeitnahen Antworten auf brennende Fragen zur Auswertung der Daten.

An das gesamte Team der interdisziplinären Arbeitsgruppe Kardiale Bildgebung des UMG Herzzentrums Göttingen geht auch ein besonderes Dankeschön. Durch die regelmäßigen Fortbildungen wurde mir ein Einblick in das Facettenreichtum wissenschaftlicher Arbeit ermöglicht und das gesamte Team stand stets für Rückfragen aller Art zur Verfügung.

Ich danke auch Frau Dr. rer. nat. A. Zapf aus der Abteilung für Medizinische Statistik für die fachkundigen Erläuterungen zur statistischen Auswertung der Daten.

Dem Deutschen Zentrum für Herz-Kreislauf-Forschung e.V. danke ich für die finanzielle Unterstützung während der Studienarbeit. 